Portland State University

PDXScholar

Fall 11-14-2014

\title{
Characterization and Modeling of Nonlinear Dark Current in Digital Imagers
}

Justin Charles Dunlap

Portland State University

Follow this and additional works at: https://pdxscholar.library.pdx.edu/open_access_etds

Part of the Physics Commons

Let us know how access to this document benefits you.

\section{Recommended Citation}

Dunlap, Justin Charles, "Characterization and Modeling of Nonlinear Dark Current in Digital Imagers" (2014). Dissertations and Theses. Paper 2073.

https://doi.org/10.15760/etd.2072

This Dissertation is brought to you for free and open access. It has been accepted for inclusion in Dissertations and Theses by an authorized administrator of PDXScholar. Please contact us if we can make this document more accessible: pdxscholar@pdx.edu. 
Characterization and Modeling of Nonlinear Dark Current in Digital Imagers

\author{
by \\ Justin Charles Dunlap
}

A dissertation submitted in partial fulfillment of the requirements for the degree of

\author{
Doctor of Philosophy \\ in \\ Applied Physics
Dissertation Committee:
Erik Bodegom, Chair
Ralf Widenhorn
Morley M. Blouke
Raj Solanki
Dean B. Atkinson

Portland State University

2014 


\begin{abstract}
Dark current is an unwanted source of noise in images produced by digital imagers, the de facto standard of imaging. The two most common types of digital imager architectures, Charged-Coupled Devices (CCDs) and Complementary MetalOxide-Semiconductor (CMOS), are both prone to this noise source. To accurately reflect the information from light signals this noise must be removed. This practice is especially vital for scientific purposes such as in astronomical observations.
\end{abstract}

Presented in this dissertation are characterizations of dark current sources that present complications to the traditional methods of correction. In particular, it is observed that pixels in both CCDs and CMOS image sensors produce dark current that is affected by the presence of pre-illuminating the sensor and that these same pixels produce a nonlinear dark current with respect to exposure time. These two characteristics are not conventionally accounted for as it is assumed that the dark current produced will be unaffected by charge accumulated from either illumination or the dark current itself.

Additionally, a model reproducing these dark current characteristics is presented. The model incorporates a moving edge of the depletion region, where charge is accumulated, as well as fixed recombination-generation locations. Recombination-generation sites in the form of heavy metal impurities, or lattice defects, are commonly the source of dark current especially in the highest producing 
pixels, commonly called "hot pixels." The model predicts that pixels with recombination-generation sites near the edge of an empty depletion region will produce less dark current after accumulation of charge, accurately modeling the behavior observed from empirical sources.

Finally, it is shown that activation energy calculations will produce inconsistent results for pixels with the presence of recombination-generation sites near the edge of a moving depletion region. Activation energies, an energy associated with the temperature dependence of dark current, are often calculated to characterize aspects of the dark current including types of impurities and sources of dark current. The model is shown to generate data, including changing activation energy values, that correspond with changing activation energy calculations in those pixels observed to be affected by pre-illumination and that produce inconsistent dark current over long exposure times.

Rather than only being a complication to dark current correction, the presence of such pixels, and the model explaining their behavior, presents an opportunity to obtain information, such as the depth of these recombination-generation sites, which will aid in refining manufacturing processes for digital imagers. 


\section{ACKNOWLEDGMENTS}

Thank you to my advisors Dr. Erik Bodegom and Dr. Ralf Widenhorn for allowing me to join them in their research and for being role models in all the various aspects of science, the scientific method, teaching, collaborating, and presenting scientific findings. Thank you to Dr. Morley M. Blouke for repeatedly demonstrating how well a skilled scientist can master their craft.

Thank you to Elliot Mylott, Will Porter, Tammy Johnson, Bradford Hill, Dave Holz, John Fastabend, Oleg Sostin, Amy Whitcombe, Jessica Sutter, Kate Brouns, Ernest Ventura, Ryan Kuroda, Dr. Michael Kriss, Ines Hartwig, Bradley Kopp, Joseph Niederriter, Bahareh Ajdari, Marc Nisenfeld, and Leroy Laush for enriching my research and my research experience.

Thank you to all of the teachers and professors I have been fortunate to have learned from and who have been kind enough to impart some of their knowledge. Thank you in particular to Dr. James Graham for first introducing me to the topics of digital imagers and dark current.

Thanks must also be given to my parents, family, friends and wife for their continued support and motivation that makes everything in my life more meaningful and enjoyable. Opportunities to formally thank them for their love and support are too few and far between. Special thanks to my wife, Alisa Dunlap, for doing all the many big things and countless little things to directly support me through my education. 


\section{TABLE OF CONTENTS}

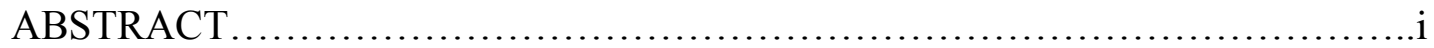

ACKNOWLEDGEMENTS........................................................ii

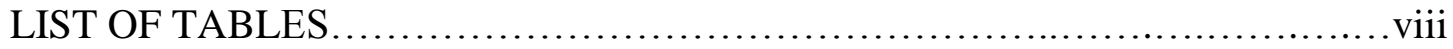

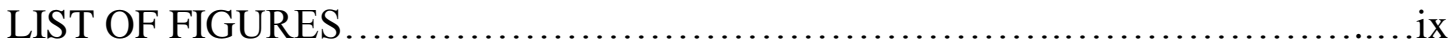

CHAPTER 1 - INTRODUCTION AND BACKGROUND INFORMATION.............1

$1.1 \quad$ Introduction.........................................................

1.2 Digital imagers......................................................

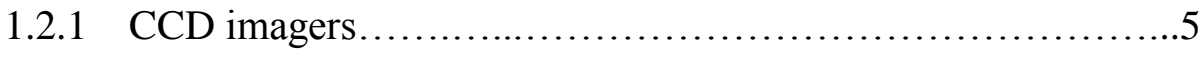

1.2.2 CMOS imagers.............................................. 8

$1.3 \quad$ Dark current........................................................

$1.4 \quad$ Dark current correction.............................................. 11

$1.5 \quad$ Activation energy calculations.................................... 14

CHAPTER 2 - INFLUENCE OF ILLUMINATION ON DARK CURRENT IN CCD

IMAGERS...........................................................

$2.1 \quad$ Introduction....................................................... 16

$2.2 \quad$ Experimental setup............................................. 17

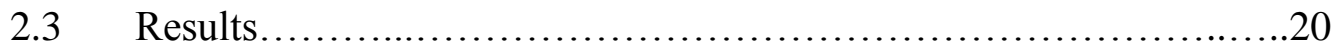


2.3.1 KAF-1602E..............................................

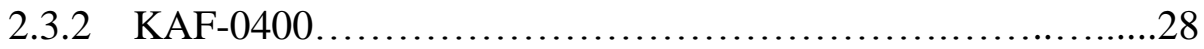

$2.4 \quad$ Influence of saturated neighboring pixels.............................33

$2.5 \quad$ Discussion...............................................................

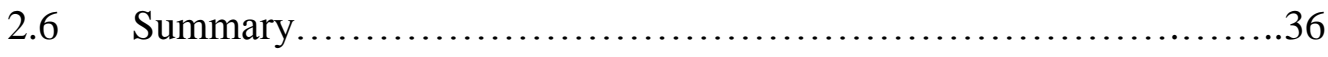

CHAPTER 3 -NONLINEAR EXPOSURE TIME RESPONSE OF CCD PIXELS ...38

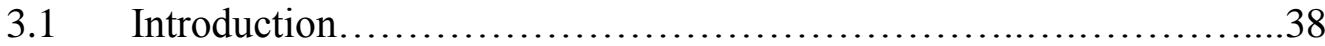

3.2 Experimental procedures and results.............................42

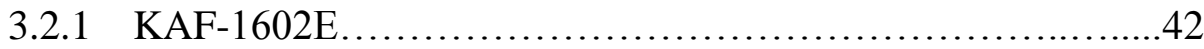

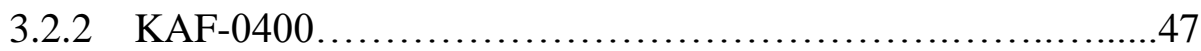

$3.3 \quad$ Discussion.................................................... 50

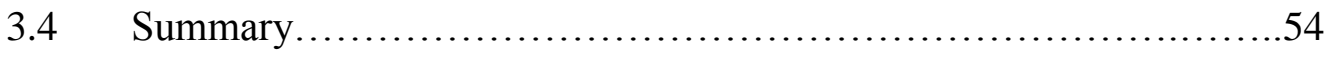

CHAPTER 4 - CMOS DIGITAL IMAGER NONLINEARITIES ...............................56

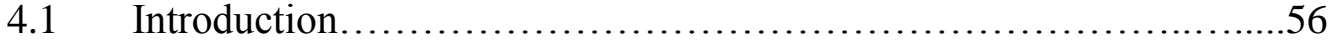

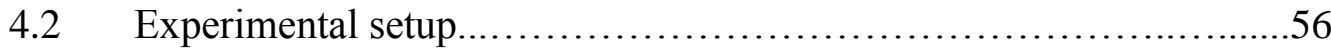

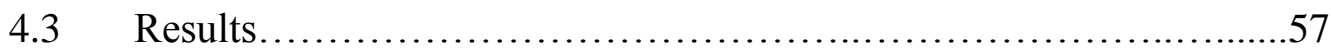

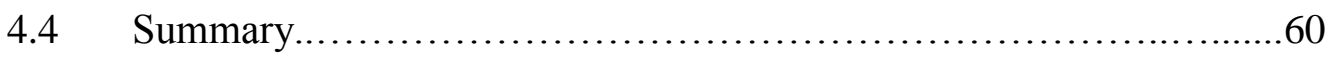

CHAPTER 5 - MODELING OF DARK CURRENT IMPURITIES AND NONLINEAR GENERATION RATES.....................................................62

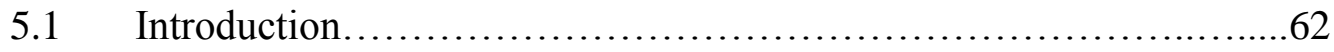

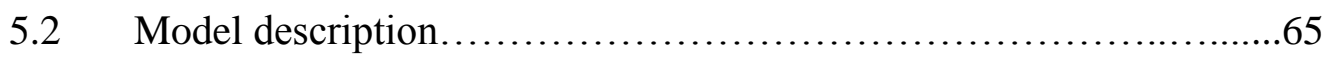


5.2.1 Dynamic depletion edge.

5.2.2 Modeling dark current production with a dynamic depletion edge .73

5.2.3 Modeled nonlinear behavior.............................76

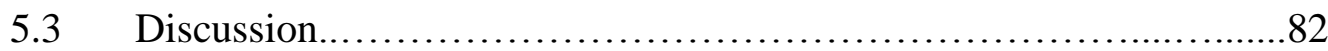

5.4 Further effects of variations in model parameters......................86

$5.5 \quad$ Conclusion................................................. 88

\section{CHAPTER 6 - MODELING OF DARK CURRENT RESPONSE TO}

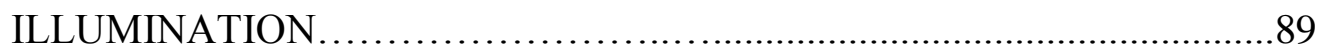

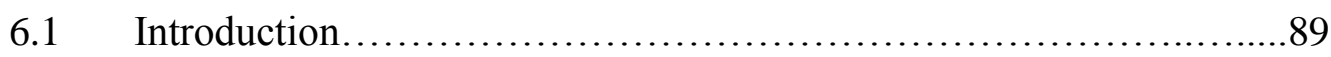

6.2 Dynamic depletion edge model..................................91

6.2.1 Model overview.......................................91

6.2.2 Modeled response to illumination..........................93

6.2.3 Modeled Nonlinear behavior............................97

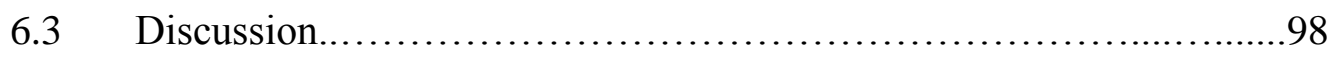

6.3.1 KAF-1602E.........................................99

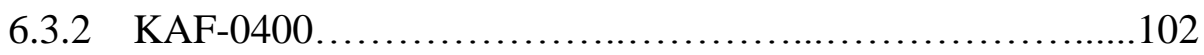

6.4 Further effects of variations in model parameters...................103

6.5 Conclusion.................................................... 104

\section{CHAPTER 7 - ACTIVATION ENERGY DEPENDENCY ON SIGNAL CHARGE}

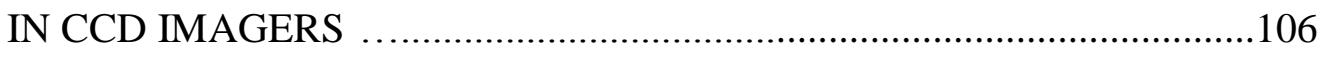

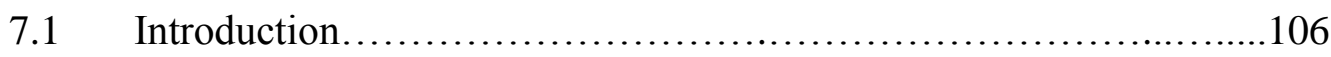

7.2 Methods and results ........................................108 
7.2.1 Traditional activation energy calculations.....................108

7.2.2 Activation energy exposure time dependency due to nonlinear production of current $\ldots \ldots \ldots \ldots \ldots \ldots \ldots \ldots \ldots \ldots \ldots \ldots \ldots \ldots \ldots \ldots \ldots \ldots \ldots 11$

7.3 Modeled activation energies due to a moving depletion edge..........115

$7.4 \quad$ Conclusion..................................................... 121

CHAPTER 8 - DISCUSSION AND CONCLUSIONS ...........................122

REFERENCES CITED............................................... 124 


\section{LIST OF TABLES}

Number

Page

Table 2.1. Camera manufacturers, models and sensor names..................17

Table 2.2. Normal dark count and change in dark count under illumination for the KAF-1602E..................................................... 21

Table 2.3. Change in dark count for the different groups under different illumination

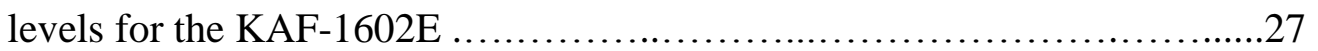

Table 2.4. Change in dark count for the different groups under different illumination

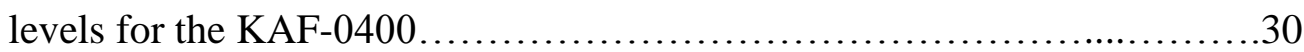

Table 5.1. Typical parameter values used by the model......................76

Table 6.1. Modeling parameters.......................................93 


\section{LIST OF FIGURES}

Number

Page

Figure 1.1: Illustration of the channel potential for a buried channel CCD pixel

Figure 1.2: Model of a three-phase CCD pixel ...........................

Figure 2.1: Graphical representation for the process of comparing dark current in frames with and without light exposures .........................19

Figure 2.2: Loss in dark count vs. light level for 6 pixels for the KAF-1602E

Figure 2.3: Distribution of the change in dark count, $\Delta D_{\text {Light }}$, at $288 \mathrm{~K}$ due to illumination for the KAF-1602E ...................................24

Figure 2.4: Distribution of the change in dark count at $278 \mathrm{~K}$ due to illumination for the KAF-0400

Figure 2.5: Loss in dark count vs. light level for 8 pixels for the KAF-0400

Figure 2.6: a) Distribution of the change in dark count due to illumination for the KAF-1602E. b) Exposure time dependence of a pixel showing negative

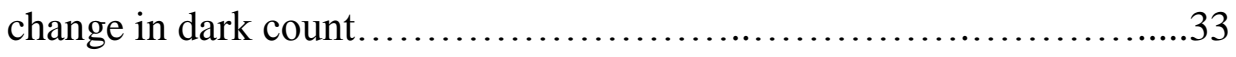

Figure 3.1: Difference of an individual dark frame and a master frame, and the individual dark frame and a scaled dark frame for the KAF-1602E.....42

Figure 3.2: Dark count at $288 \mathrm{~K}$ as a function of exposure time for 5 pixels for the KAF-1602E.

Figure 3.3: Dark current from linear fit versus the change in dark count, $\Delta D$, for the KAF-1602E 45

Figure 3.4: Dark current from linear fit versus the change in dark count, $\Delta D$, for the KAF-0400 ...............................................48

Figure 3.5: Dark count at $278 \mathrm{~K}$ as a function of exposure time for eight pixels for the KAF-0400.............................................50 
Figure 3.6: Change of dark count after illumination versus dark count of a $600 \mathrm{~s}$

dark frame.

Figure 4.1: Change in dark current $(\triangle D)$ between slopes $(m)$ after $5 \mathrm{~s}$ extrapolated out to 50s (or to a fixed maximum count of the nearest frame to 1800 counts) at $278 \mathrm{~K}$. .58

Figure 4.2: Dark count at $278 \mathrm{~K}$ as a function of exposure time for seven pixels for the CMOSIS. .59

Figure 4.3: Dark count at $278 \mathrm{~K}$ as a function of exposure time for five hot pixels from the CMOSIS imager with variation in saturation level...........60

Figure 5.1: Dark count at $288 \mathrm{~K}$ as a function of exposure time for 5 pixels for the KAF-1602E sensor

Figure 5.2: Moving depletion region in a CCD pixel .65

Figure 5.3: Dopant concentration as a function of depth from the surface of the

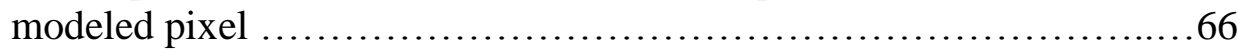

Figure 5.4: Modeled dark current for $1 \times 10^{6}$ pixels from a linear fit over the first $50 \mathrm{~s}$ versus the change in dark count, $\Delta D$, evaluated at $1500 \mathrm{~s} \ldots \ldots \ldots \ldots . . .78$

Figure 5.5: Schematic diagrams of possible cases for depths of impurities in modeled pixels

Figure 5.6: Dark count as a function of exposure time for 8 modeled pixels

Figure 5.7: (a) Dark current from linear fit versus the change in dark count, $\Delta D$, for the KAF-1602E evaluated at $1800 \mathrm{~s}$, (b) modeled data resulting in a poor fit due to using a $\delta$ value of $3.0 \times 10^{-5} \mathrm{~cm}$, (c) modeled data with a good fit using a delta value of $3.0 \times 10^{-6} \mathrm{~cm} \ldots \ldots \ldots \ldots \ldots \ldots \ldots \ldots \ldots . \ldots \ldots$

Figure 5.8: Modeled dark current from linear fit versus the change in dark count, $\Delta D$, with (a) 10,000 impurities, and (b) 100,000 impurities

Figure 5.9: Dark current from linear fit versus the change in dark count, $\Delta D$, modeled with a gate voltage of (a) $-5 \mathrm{~V}$, and (b) $0 \mathrm{~V}$ .88

Figure 6.1: Two-dimensional representation of the one-dimensional pixel with an impurity near the moving depletion edge 
Figure 6.2: Change of dark count after illumination versus dark count of a $500 \mathrm{~s}$ dark frame with an initial count of $30,000 \ldots \ldots \ldots \ldots \ldots \ldots \ldots \ldots \ldots . . . \ldots 94$

Figure 6.3: Modeled dark current for $1 \times 10^{6}$ pixels from a linear fit over the first $50 \mathrm{~s}$ versus the change in dark count, $\Delta D$, evaluated at $1800 \mathrm{~s} . \ldots \ldots \ldots . . . .98$

Figure 6.4: Change of dark count after illumination versus dark count of a $600 \mathrm{~s}$ dark frame. (a) KAF-1602E for an average initial count due to the light exposure of approximately 26,000 at $288 \mathrm{~K}$. (b) modeled data...........100

Figure 6.5: Change of dark count after illumination versus dark count of a $600 \mathrm{~s}$ dark frame. (a) KAF-0400 for an average initial count due to the light exposure of approximately 42,000 at $278 \mathrm{~K}$. (b) modeled dark current.

Figure 6.6: Change of dark count after illumination versus dark count of a $500 \mathrm{~s}$ dark frame. (a) Modeled using 10,000 impurities distributed throughout the imager. (b) Modeled using 100,000 impurities distributed throughout the imager.

Figure 6.7: Change of dark count after illumination versus dark count of a $500 \mathrm{~s}$ dark frame. Modeled dark current with a gate voltage of (a) $-5 \mathrm{~V}$ and (b) $0 \mathrm{~V}$ 104

Figure 7.1: Arrhenius plots of single hot pixels, including (a) a pixel showing no deviation from linearity at exposure times of $240 \mathrm{~s}$ and $960 \mathrm{~s}$, as well as (b) and (c) two pixels showing deviation from linearity at high temperatures

Figure 7.2: Activation energies calculated at a series of exposure times for the KAF-1602E sensor ...

Figure 7.3: Dark count at $288 \mathrm{~K}$ versus exposure time for the KAF-1602E sensor

Figure 7.4: Activation energy versus constant count values.

Figure 7.5: Modeled counts vs. exposure time for simulated pixels with a moving depletion edge and fixed impurities.

Figure 7.6: Modeled Arrhenius plots with a 900 s exposure time. .118

Figure 7.7: Modeled calculated activation energies at varying exposure times. 
Figure 7.8: Modeled Arrhenius plots with a fixed value of 5000 DN........... 120

Figure 7.9: Modeled calculated activation energies at varying fixed counts......................................................... 120 


\section{CHAPTER 1 - INTRODUCTION AND BACKGROUND INFORMATION}

\subsection{Introduction}

Digital imagers are all but ubiquitous in the world of imaging, with silver halide filmbased cameras being used and manufactured at an ever declining rate. Meanwhile, digital imager use is expanding into more and more applications and markets due to their compact size, inexpensive production costs, and easily transferable image files. Digital imagers are primarily of two types, the Charge-Coupled Device (CCD) or the Complementary Metal-Oxide-Semiconductor (CMOS). While the merits of digital imagers over film are many, there are some drawbacks to digital imagers including the presence of thermally dependent noise, commonly referred to as dark current, found in both $\mathrm{CCD}$ and CMOS based digital imagers. This noise source limits the dynamic range of the final light image and, due to its variability from pixel to pixel, creates Fixed Pattern Noise (FPN) across the imager that will partially mask the light signal if not removed.

Analyzing and characterizing dark current generation can lead to methods for correction as well as methods for improving manufacturing of semiconductor devices in general and digital sensor design in particular. The modeling methods described and used in this dissertation may be used as a means for determining depths of recombination-generation sites, e.g. heavy metal impurities. This could help in 
determination of when the impurities are introduced and which step of manufacturing can be improved.

The scope of this dissertation is to document the behavior of atypical dark current observed in particular pixels in digital imagers. In Chapter 2, a study detailing the effect of illumination on dark current for CCD pixels is described. In particular, presented are data indicating that a subset of pixels produce less dark current after being exposed to illumination. Further shown, in Chapter 3, is that dark current in these pixels displays a nonlinear dependence on exposure time. This behavior is a complication to the standard methods of noise correction, where dark current is expected to be linear with exposure time and unaffected by illumination, but may be a way to locate the physical position of the defect that is the source of the dark noise. In Chapter 4 , it is shown that this behavior is constrained to $\mathrm{CCD}$ pixels and that a CMOS imager contains pixels showing similar nonlinear dark current production with respect to exposure time. In Chapters 5 and 6, a model is presented utilizing fixed impurity locations and a moving depletion edge. The model is successful in reproducing much of the behavior observed by these pixels. Chapter 7 details one of the complications to analyzing and characterizing pixels prone to this nonlinear production of dark current. In particular, that activation energy calculations are sensitive to the parameters, such as exposure time, chosen in obtaining data from a sensor. This is a complication that is not conventionally dealt with, as well-behaved pixels are not sensitive to these parameters, however it is the pixels that are not well behaved that the activation energies are the most interesting. 


\section{$1.2 \quad$ Digital imagers}

The invention of the Bucket Brigade Device by Sangster and Teer in 1969 [1], and the improvement of the concept into the invention of the CCD by Boyle and Smith in October of 1969[2], laid the technical foundation for digital imagers. However, they were not quickly adopted because semiconductor technology had not advanced to a sufficient point to make digital imagers cost effective or competitive in terms of quality with film imagers. Regardless, the search for a smaller and lighter imager with lower noise and a larger dynamic range to replace the existing technology of tube-type detectors was instrumental in advancing CCD technology. This search primarily came from the space imaging sector and the NASA mission to build what is now known as the Hubble Space telescope [2]. CCDs quickly gained favor in the space imaging sector and, along with the competing CMOS format, have all but replaced any other competing method of imaging.

A discussion of digital imagers requires a basic knowledge of the transfer of energy from photons to charge carriers in the substrate of the imager. Incident photons will be absorbed by the substrate of the imager if they have energy, equal to Planck's constant times the frequency of the radiation, $h v$, greater than the bandgap energy, $E_{g}$, of the semiconductor. In such an event, an electron will be excited from the valence band to 
the conduction band and thus an electron-hole pair will be created in the bulk of the semiconductor. The charge carriers can then be collected by establishing an electric field within the substrate of the imager. Such an electric field is set up in the imager using gates at differing voltages so as to make a potential well. Shown in Fig. 1.1 is a potential well created after applying a voltage, $V_{g}$, to a gate. This potential well, also called the depletion region, will collect any photo-electrons generated in the region as well as a percentage of the electrons generated nearby in the neutral regions of the semiconductor that then diffuse into the depletion region. This phenomenon will continue throughout the collection period, often referred to as the exposure time, the length of time that the imager is exposed to light. The total output current, $I_{\text {out }}$, collected by each pixel is then simply given by the following equation:

$$
I_{o u t}=\frac{q_{n}}{t_{\text {int }}}
$$

where $q_{n}$ is the total charge collected by the pixel, and $t_{\text {int }}$ is the integration time [1].

However, electrons may also be thermally excited into the conduction band within the substrate, and if this event occurs nearby or within the depletion region, the electrons created in this process may also be collected in the depletion region. The electrons collected in this manner are often referred to as dark current and will be discussed in greater detail in Section 1.3.

The collected electrons are then read out by the imager. Measurement of the stored charge in a CCD is most often done by measuring the change in voltage of the channel 
$\left(\Phi_{C H}\right)$ caused by the potential well filling with charge carriers. Details of the exact process vary between imager designs; however, it is important to the discussion of noise to note that standard CCDs are passive in the sense that the photo-generated charge is not amplified prior to being forwarded on to an output amplifier, whereas CMOS pixels are generally active, meaning that amplification is done within the pixel itself. Additionally, a single CMOS pixel can be accessed using an X-Y address scheme whereas CCDs require entire rows to be read out at a time.
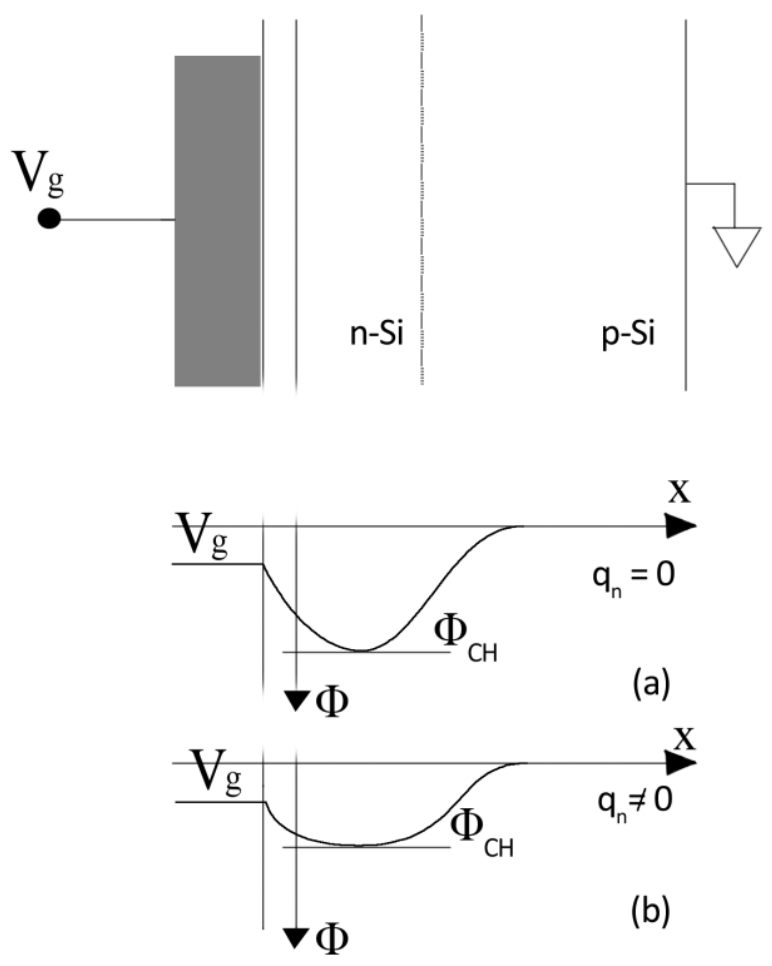

Fig. 1.1. Illustration of the channel potential for a buried channel CCD pixel with empty (a), and filled (b) wells. (Image based on a drawing in "SolidState Imaging with Charge-Coupled Devices" by Theuwissen)[1]

\subsubsection{CCD Imagers}


CCD digital imagers are constructed of a two-dimensional matrix of light-sensitive Metal Oxide Semiconductor (MOS) capacitors in a configuration so as to create individual pixels. Many such pixels comprise a digital imager. Increasing numbers of pixels are a selling point for cameras and imagers imbedded within smart phones, with modern designs for consumer chips commonly incorporating around ten million pixels. Depicted in Fig. 1.1 is a cross sectional graphical representation of a threephase CCD pixel consisting of a conductive gate, a semiconductor substrate, and thin layer of an oxide semiconductor with a large bandgap preventing flow of charge carriers between the gate and the substrate. A positive voltage applied to a gate drives the holes away from the surface and leaves behind negatively charged acceptor atoms, thus leaving behind a region depleted of mobile carriers. This region is called the depletion region [2].

During the collection phase of the imaging process, incoming light strikes the imager and excites electrons from the valence into the conduction band within the substrate. These photogenerated electrons are collected by the potential well as shown in Fig. 1.1. Based on the application of various voltages to the three polysilicon gates (labeled as A, B, and $\mathrm{C}$ in Fig. 1.2), the depletion region is formed within the bulk silicon below the gates. An adroit altering of the voltages will modify the depletion region and will allow for the well, and therefore also the charges contained within it, to be shifted to either side. As a possible example, during the collection phase the voltage applied to the A gate is higher than either B or C (as shown) and therefore, the 
depletion region is formed beneath the A gate. To shift the charges, a higher voltage can be applied to the B gate, while slowly decreasing the voltage across the A gate, ultimately shifting all the stored charges from beneath A to beneath B. Then, in a similar fashion, a high voltage is applied to the $\mathrm{C}$ gate while decreasing the $\mathrm{B}$ voltage to shift the charges to lie beneath the $\mathrm{C}$ gate. Continued alterations of the voltages in this way allow for charges to be completely shifted to where they can be read out. An image can be formed by orderly reading out the stored charge for all the pixels in the array [3].

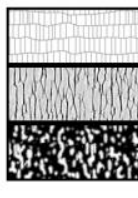

Polysilicon gates

Insulation layer between gates

Gate oxide

Depletion region between boundary

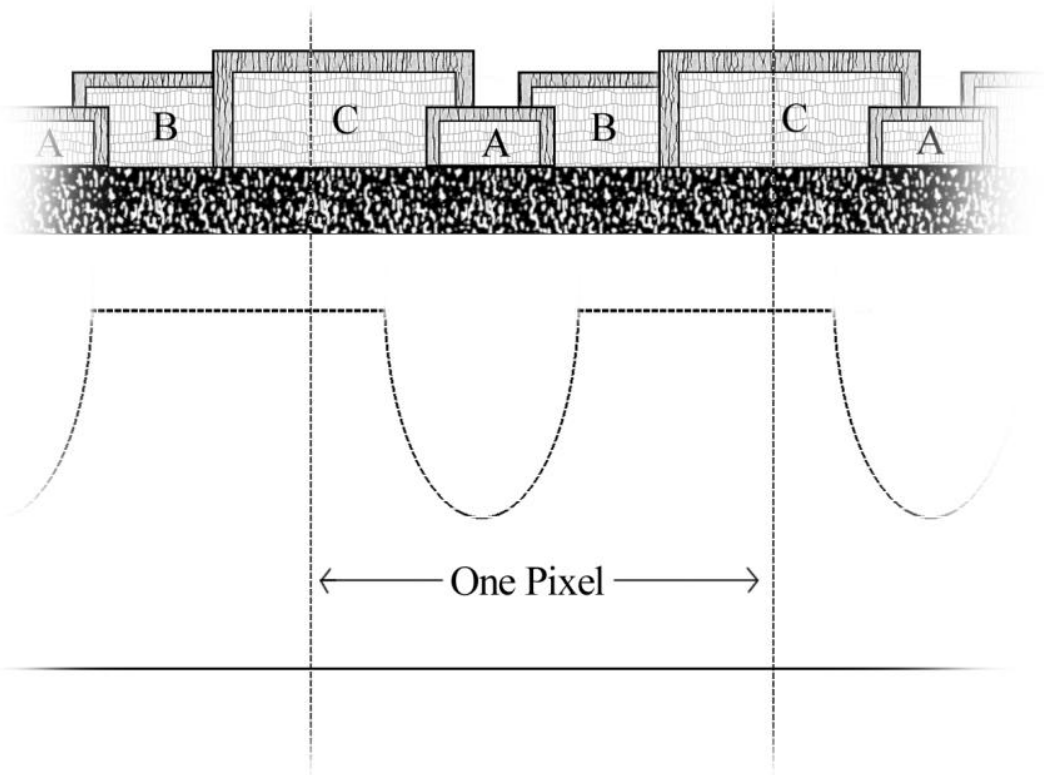

Fig. 1.2. Model of a three-phase CCD pixel. (Image based on a drawing in "CMOS Imagers: Phototransduction to Image Processing" edited by Yadid-Pecht and Etienne-Cummings)[3] 


\subsubsection{CMOS imagers}

The concept of the CMOS imager using Active-Pixel Sensor (APS) technology was introduced in the early 1990s but initially suffered inferior performance due to large levels of dark current. However, CMOS imagers have improved significantly from when they were first produced and have proven to consume less power than CCDs as well as have higher pixel readout rates [3]. Due to similarities with processes in the fabrication of other semiconductor components, CMOS imagers have also been cheaper to produce. Therefore, they first found increased market share in applications that could afford lower quality images and required less expensive sensors such as in cellular phones.

Many of the improvements in the more mature CCD fabrication technology, including micro-lenses, have been successfully borrowed to improve CMOS sensors. Due to improvements in their quality, CMOS sensors are found in all range of products and rival CCDs in most markets [3].

However, at this time there is no clear winner between the two competing technologies. Both technologies have their advantages. Among the advantages of CCD sensors are: a larger area of the pixel is reserved for light collection as a result of having fewer elements located in the pixel itself, their quantum efficiency can approach $90 \%$, and CCDs have less FPN as a result of APS imagers having active transistors and therefore also unique noise sources located in each of the pixels. While 
this type of noise can be partially removed by correlated double sampling, in the process it also introduces column-to-column FPN that must then itself be removed itself by other methods [3].

Despite their differences, both technologies share many things in common and are based on the same fundamentals of solid state imaging which, unfortunately, also means they share the side-effect of generating and collecting thermally induced electrons.

\subsection{Dark current}

One of the primary selling points of new digital cameras has been the imager's total number of pixels. While more pixels do produce an image with higher resolution and generally a better picture, there are costs to fitting more pixels on the same size imager. With a greater number of pixels and a fixed imager size, the individual pixels must correspondingly decrease in size. Unfortunately, the pixel sensitivity and fullwell capacity decreases with pixel size [4]. Full-well capacity, $N_{\text {sat }}$, also known as saturation, refers to the total amount of charge that can be accumulated on a photodiode capacitor and is given (in electrons) by the equation:

$$
N_{\text {sat }}=\frac{1}{q} \int_{V_{\text {reset }}}^{V_{\max }} C_{p d}(V) d V
$$

where $C_{p d}$ is the photodiode capacitance and $q$ is the charge of an electron. The maximum voltage, $V_{\max }$, and initial voltage, $V_{\text {reset }}$, are dependent upon the photodiode structures and the operating conditions [3]. Excluding all other noise sources except 
for the photon shot-noise, which is due to the statistical nature of photons and is characterized by the square root of the signal, the maximum signal-to-noise ratio (SNR) is determined by the full-well capacity. However, any additional noise source decreases the SNR and also decreases the potential dynamic range (defined as the ratio between the full-well capacity and the noise floor) of the image. The noise floor (also called read noise) is noise from the readout electronics.

A primary source of noise in an imager is dark current, an unwanted strongly temperature-dependent signal that is a result of lattice defects or metal impurities (such as iron or gold) in the silicon lattice of the imager [2]-[9]. An impurity or lattice defect creates an alternative pathway for electrons excited by heat within the imager to leave the valence band and reach the conduction band. In effect, less energy is needed to excite these electrons, and once in the conduction band the electrons can be collected in the depletion region, therefore partially filling the potential well.

Dark current is primarily a problem when there is a low level of light signal and the dark current rivals that of the current generated by the light. The accumulation of dark current over larger integration times limits the SNR. This is especially a concern for astrophotography and night photography, however any image taken at high temperatures or under conditions that necessitate a long exposure time or high ISO camera setting are strongly affected by the presence of dark current. In addition, dark current varies from pixel to pixel on the imager due to variations in defects and 
impurities, creating the largest source of FPN. Exceedingly hot pixels, pixels that show a much larger dark current than their neighbors, can be visually apparent even at less extreme conditions.

\subsection{Dark current correction}

Due to the degradation of the image by dark current, many methods have been devised to limit its effects including the development of changes in manufacturing, processing done by the camera to mask the hot pixels, and the removal of the dark signal by taking images without a light source that are subtracted from the light image.

Many changes to the design of digital imagers have been focused on limiting dark current. This has included the introduction of a buried channel CCD design that moves the depletion region away from the $\mathrm{Si}-\mathrm{SiO}_{2}$ interface as this surface is more prone to having impurities or defects. This largely removes the surface dark current, but dark current still exists in the form of depletion current, current generated within the depletion region itself, and diffusion current, current generated outside the depletion region but that ultimately diffuses into and is subsequently collected by the depletion region.

In an effort to remove and hide the dark current that remains in the imagers, camera manufacturers have devised further methods. For instance, some imagers include pixels that are not exposed to light, and act as a means for the camera to measure the 
base line of the dark current in the rest of the imager that can be subtracted from the final image. However, this method does not adequately take into account the FPN nature of dark current nor the shot noise of each individual pixel. In addition, some manufacturers appear to use a median filter to remove especially hot pixels [10], [11]. This method removes the signal due to the offending pixel by averaging the pixel's neighbors and substituting that value in place of the value measured by the pixel itself. This removes noise created by the hot pixel, but also removes any possible signal gathered by that pixel and results in a net loss of information.

Once an imager is manufactured, and assuming that no processing is done to the image by the camera, there exists dark current FPN that is unique to the imager, that does not significantly change with time, and is present in any image taken by that imager. In other words, an image taken under the same conditions (same temperature, exposure time, and ISO setting), will have roughly the same amount of dark noise as another image taken at any other time. However, there are two exceptions to this otherwise consistent behavior. First, the imager may acquire additional defects due to events such as exposure to radiation that will change the FPN with time [12]. Second, generation of dark current is a statistical process, much like the generation of photons, and therefore will vary about an average according to Poisson statistics. Thus, a signal, either that of light or that of dark current, will vary with a SNR equal to the square root of the signal. This variation is called the shot noise and creates an intrinsic randomness in the amount of dark current collected for any given set of conditions. 
The most common method of removing dark current from a light image is to subtract a second image that is taken with a closed shutter. This so-called dark frame, most commonly taken after the light image, can then be subtracted from the light image and, ideally, removes the dark signal leaving only the signal from incoming photons. It appears that this is the method that most consumer camera manufactures use for images with sufficiently long exposure times or high ISO settings. For instance, it was found for an image taken with an exposure time of $1.3 \mathrm{~s}$ or greater, Canon cameras display a waiting symbol and are unresponsive for an amount of time slightly greater than the exposure time; time likely spent taking the dark frame and subtracting it from the initial frame [11].

To partially account for the shot noise of the dark current it is also common, especially in astrophotography, to take and average a series of dark frames, thus creating a master dark frame that has a higher SNR than a single image itself. A similar method that can be effective for imagers with a sufficiently large full-well capacity, is to take a dark frame that is significantly longer than needed and then scale that dark frame back to the exposure time needed to subtract from the light image. A dark frame created by either of these methods can then be subtracted from the light exposure in the same way a single frame can be. However, an unfortunate requirement of both these methods is the additional time needed to generate the dark frame. 


\subsection{Activation energy calculations}

A common method of analyzing dark current is by using the Arrhenius equation:

$$
X=X_{0} \exp \left(-E_{A} / k T\right)
$$

to calculate the activation energy $E_{A}$. In this equation $X$ is a measured property, $X_{0}$ is the exponential prefactor, $k$ is Boltzmann's constant, and $T$ is the temperature [13]. This empirical law was characterized in 1884 by J.H. van't Hoff, but received its name from the Swedish chemist Svante Arrhenius who provided an explanation for the possible mechanism of the law in chemical reactions [14]. The law is generally used to characterize chemical reactions that are temperature dependent. Additionally, the law can be used to characterize dark current performance in digital imagers where now we can write the Arrhenius equation to explicitly reference dark current generation as the measured property:

$$
D=D_{0} \exp \left(-E_{A} / k T\right)
$$

In this equation, $D$ is the generation rate of dark current and $D_{0}$ is the exponential prefactor.

In previous studies such as [15] it has been observed that under some circumstances the prefactor $D_{0}$ will further obey the Meyer-Neldel rule (MNR) such that:

$$
D_{0}=D_{00} \exp \left(-E_{A} / k T_{M N}\right)
$$

where $D_{00}$ is a positive constant and $T_{m n}$ is the iso-kinetic temperature, or the temperature at which reactions in different pixels would all proceed at the same rate [16]. This rule, also known as the "compensation law", has been given as an empirical 
result since 1937 [13]. Work done by Widenhorn et al. in [15] demonstrates that the MNR is only valid over a small temperature range and does not apply in the regime where $\mathrm{T}$ approaches $T_{m n}$. Further, their work indicates that the generation of dark current in semiconductor pixels can be explained through the contributions of two distinct dominant processes, diffusion and depletion dark current. The depletion dark current is generated in the depletion region of the pixel itself and is dominant at relatively cooler temperatures, approximately room temperature and below. These temperatures are generally where it is assumed digital imagers will be operated. The diffusion dark current is generated in the field-free region of the bulk silicon and is dominant at relatively warmer temperatures. Assuming these are the two dominant contributions of dark current, they show that the measured dark current can be expressed such that:

$$
D=D_{0, d i f f} T^{3} \exp \left(-E_{g} / k T\right)+D_{0, d e p} T^{3 / 2} \exp \left(-E_{g} / 2 k T\right)
$$

where $E_{g}$ is the bandgap of $\mathrm{Si}$ which is temperature dependent itself.

Therefore it is expected that the activation energy, when the generated dark current in the depletion region and/or at the surface are dominant, will be measured to be approximately half the bandgap. When the diffusion dark current is dominant, the activation energy should be closer to the activation energy of the bandgap. 


\section{CHAPTER 2 - INFLUENCE OF ILLUMINATION ON DARK CURRENT IN CCD IMAGERS}

\subsection{Introduction}

In this section, we address the question of whether the dark current produced in an image taken with a closed shutter is identical to the dark current produced in an exposure in the presence of light. In our investigation, we illuminated two different CCD chips to different intensities of light and measured the dark current generation. A surprising result of this study is that some pixels produce a different amount of dark current under illumination. Finally, we discuss the implication of this finding for dark frame image correction. Much of the material covered in this chapter has been published in [17].

A basic understanding of dark current in a CCD can be gained by analyzing the dark current generated in the p-n junction of a diode [18]-[22]. It has been shown that the non-uniformity in the dark count is caused by deep level impurities like $\mathrm{Au}, \mathrm{Fe}$, or $\mathrm{Ni}$ [6], [8], [9], [23], [24]. The fixed pattern of the dark count can be corrected by subtracting a dark frame. A dark frame is generated by taking an image under the same condition as the light exposure (equal exposure time and temperature), with the difference that the shutter remains closed. To decrease the dark current shot noise, often multiple dark frames are taken and averaged to generate a master frame. If one does not have the time to obtain a master dark frame for each imaging session, one can also calibrate the dark current of an imager. After the imager is calibrated for a certain 
temperature and exposure time range one can compute a dark frame almost instantly [25].

Every dark current subtraction assumes that the thermal generation of electrons is identical under illuminated and non-illuminated condition. It is tacitly assumed that the dark current generation is not affected by the presence of light or by the electrons that have already been optically excited and collected in the well.

\subsection{Experimental setup}

For our study we used two different cameras; the SBIG ST-8XE with a KAF-1602E CCD sensor and the Meade Pictor 416XT with a KAF-0400 CCD sensor. Both CCD chips are buried-channel frontside-illuminated CCDs. The KAF-1602E is a $1530 \mathrm{x}$ 1020 pixel array with an individual pixel size of $9 \mu \mathrm{m} \times 9 \mu \mathrm{m}$. The KAF-0400 has the same individual pixel size, but only 768 x 512 pixels. Both cameras are scientific cameras and are frequently used by astronomers. The camera manufacturer, camera model, and sensor names are used interchangeably throughout this dissertation, with an attempt at using the most fitting designation depending on the context. Table 2.1 is presented for reference.

\begin{tabular}{|c|c|c|}
\hline Camera Manufacturer & Camera Model & Sensor Designation \\
\hline SBIG & ST-8XE & KAF-1602E \\
\hline Meade & Pictor 416 XT & KAF-0400 \\
\hline
\end{tabular}

Table 2.1. Camera manufacturers, models and sensor names. 
We placed a LED operated with a constant current supply at a distance of about 10 to $20 \mathrm{~cm}$ from the camera so that the whole chip was illuminated approximately uniformly.

In order to control the influx of light during an exposure an external shutter was mounted in front of the camera. The internal camera shutter was opened during all exposures. The external shutter was opened for 100 to $800 \mathrm{~ms}$ of "pre-illumination" within the first $5 \mathrm{~s}$ of the exposure. Only for the short time that the external shutter is opened did light reach the CCD. After the external shutter was closed, the remaining exposure was done in the "dark." The light level was controlled by varying the opening time of the external shutter, the current through the LED, and the distance between camera and light source. Immediately following each light exposure, a regular dark frame with the same exposure time was taken again with the internal shutter open and the light on, but this time the external shutter remained closed throughout the whole exposure. Figure 2.1 illustrates the process and the images taken for obtaining the change in dark count between frames with and without light exposure. 


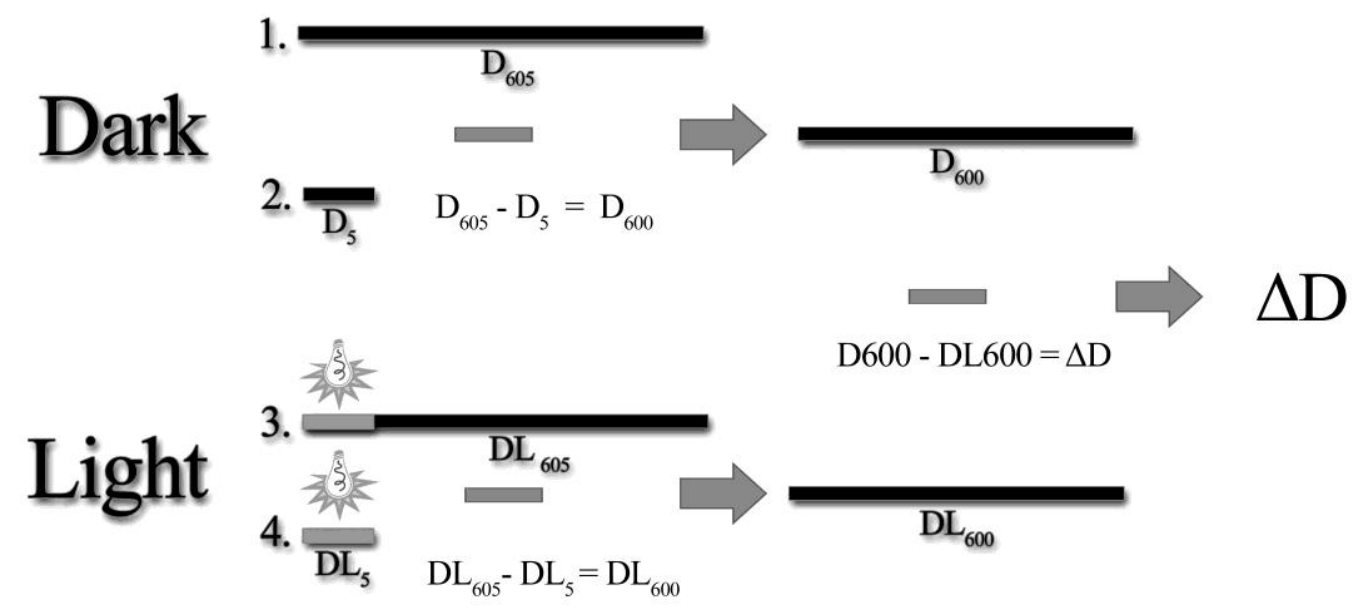

Fig. 2.1: Graphical representation for the process of comparing dark current in frames with and without light exposures.

To measure the dark current over an integration time of $600 \mathrm{~s}$ with and without light, we took the following images.

1. $D L_{605}$ : Frame with the presence of pre-illumination, $605 \mathrm{~s}$ total exposure time, external shutter opened at the beginning of the exposure for 100 to $800 \mathrm{~ms}$

2. $D L_{5}$ : Frame with the presence of pre-illumination, $5 \mathrm{~s}$ exposure time, external shutter opened at the beginning of the exposure for 100 to $800 \mathrm{~ms}$

3. $D_{605}:$ Dark frame, $605 \mathrm{sec}$, closed external shutter

4. $D_{5}$ : Dark frame, $5 \mathrm{sec}$, closed external shutter

The pre-illumination exposure at the beginning is the same for the $D L_{605}$ and the $D L_{5}$ frames and is subtracted out by taking the difference of the two exposures. Therefore, the dark current, $D L_{600}$, accumulated during $600 \mathrm{~s}$ after the image was initially exposed to light is given as:

$$
D L_{600}=D L_{605}-D L_{5}
$$


The dark current, $D_{600}$, accumulated during $600 \mathrm{~s}$ while the camera was not exposed to light, is given as:

$$
D_{600}=D_{605}-D_{5}
$$

We will refer to this parameter, $D_{600}$, as the normal dark count, since this is the count obtained in a regular $600 \mathrm{~s}$ dark frame.

To compare the dark count accumulated in $600 \mathrm{sec}$, one subtracts the dark count without light from the dark count with light:

$$
\Delta D_{\text {light }}=D_{600}-D L_{600}
$$

For each exposure type and time, 20 and 25 images were taken for the KAF-0400 and KAF-1602E, respectively. The data presented here are the average values of those images. All data is expressed in counts, the number of electrons for each count can be obtained from the gain of each camera. For the KAF-1602E the gain is $2.3 \mathrm{e}^{-/} \mathrm{DN}$ and for the KAF-0400 the gain is $1.2 \mathrm{e}^{-/} \mathrm{DN}$. The full well of KAF-1602E and the KAF0400 was 43,500 counts and 85,000 counts, respectively. All data presented here is below the saturation level of the chip.

\subsection{Results}

\subsubsection{KAF-1602E}

One generally assumes that dark current is independent of the light intensity. Under this assumption the dark current for the $D L_{600}$ and the $D_{600}$ frames should be the same and $\Delta D_{\text {light }}$ approximately zero. However, this is not true for all pixels and light 
intensities. Figure 2.2 shows the change in the dark count, $\Delta D_{\text {light }}$, as a function of the average count of the initial $5 \mathrm{~s}$ light exposure for six pixels. Pixels 4 and 5 do not show a significant change in the dark count, independent of the pre-illumination. For Pixels 2, 3a, and 3b, the dark count decreases with increasing level of illumination.

The decrease in dark current slowly levels off for higher illumination levels. Pixel 1 also shows a decrease in dark count. Its decrease in dark count is roughly constant for initial light levels of 8,000 counts or more.

\begin{tabular}{|c|c|c|c|c|c|c|}
\hline Pixel & Group & $\begin{array}{c}\mathrm{D}_{600} \text { in } \\
\text { counts }\end{array}$ & $\begin{array}{c}\Delta D_{\text {light }} / D_{600} \\
\text { at 2,000 } \\
\text { counts }\end{array}$ & $\begin{array}{c}\Delta D_{\text {light }} / D_{600} \\
\text { at } 8,500 \\
\text { counts }\end{array}$ & $\begin{array}{c}\Delta D_{\text {light }} / D_{600} \\
\text { at } 16,000 \\
\text { counts }\end{array}$ & $\begin{array}{c}\Delta D_{\text {light }} / D_{600} \\
\text { at } 26,000 \\
\text { counts }\end{array}$ \\
\hline 1 & 1 & 1,139 & $60.4 \%$ & $83.8 \%$ & $82.1 \%$ & $85.6 \%$ \\
\hline 2 & 2 & 5,956 & $0.8 \%$ & $5.2 \%$ & $11.9 \%$ & $22.2 \%$ \\
\hline $3 a$ & $3 a$ & 2,716 & $7.5 \%$ & $24.0 \%$ & $39.0 \%$ & $51.7 \%$ \\
\hline $3 b$ & $3 b$ & 2,846 & $3.2 \%$ & $12.4 \%$ & $23.9 \%$ & $37.1 \%$ \\
\hline 5 & Major Peak & 184 & $-4 \%$ & $-5 \%$ & $10 \%$ & $-5 \%$ \\
\hline
\end{tabular}

Table 2.2. Normal dark count and change in dark count under illumination at the indicated counts for the KAF-1602E. 


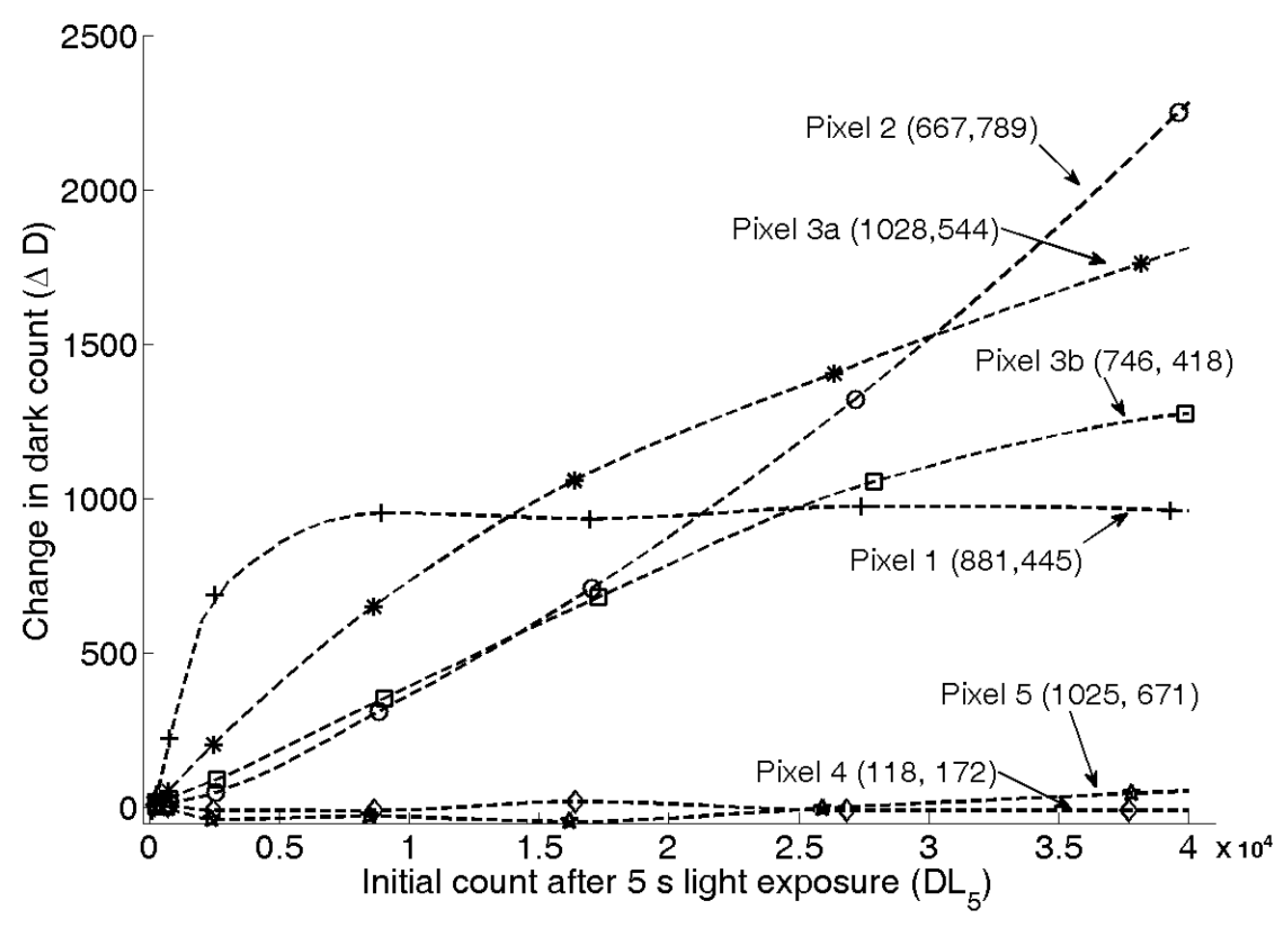

Fig. 2.2: Loss in dark count vs. light level for 6 pixels for the KAF1602E. The dark count in the light exposures decreases with increasing light level. The numbers in the brackets are the $\mathrm{x}$ and $\mathrm{y}$ coordinates of the pixel.

The normal dark count for the pixels in Fig. 2.2 are presented in Table 2.2. At $288 \mathrm{~K}$ the average dark count across the chip without the presence of light, $D_{600}$, for a $600 \mathrm{~s}$ exposure is 397 counts. Except for Pixel 4, all pixels in Fig. 2.2 are hot pixels, that is, the dark count is much larger than the average dark count of the image sensor. As shown later, each pixel represents a group of pixels with a distinct behavior under illumination.

A two-dimensional distribution of the loss in dark count, $\Delta D_{\text {light }}$, versus the dark count level, $D_{600}$, was created. The $\Delta D_{\text {light }}$ vs. $D_{600}$ plane was divided into intervals of size 
50 counts by 50 counts and then, for each pixel according to its dark count values, the frequency for the corresponding interval is incremented by one. Figure 2.3 shows the resulting distributions for five different illumination levels. The shades of grey show how many pixels were in a specific interval. Due to the wide range of frequencies the grey scale is represented as the logarithm of the frequency.

All five panels in the figure show a characteristic form that gets more defined with increase of initial illumination level. For a specific dark count level, only very specific ranges of values for the change in dark count were observed. Based on the decrease in dark count, we separated those pixels into different groups.

A large number of pixels generate a small quantity of dark current and the light exposure does not change this behavior. These pixels are seen in Fig. 2.3c close to the origin of the figure with small or no change in the dark current (Main Peak). Approximately 1.5 million or $97 \%$ of all pixels behave this way (see also Table 2.2). Pixel 4 in Fig. 2.2 is one example of a pixel located in the Main Peak. Its normal dark count is about 184 counts and the change for all illumination levels is smaller than 20 counts. Pixel 5 in Fig. 2.2 is a pixel that is located in a second smaller peak of the distribution in Fig. 2.3. The approximately 16,600 pixels in this group have a large dark count, $D_{600}$, of about 6,000 counts but show no change under illumination. 

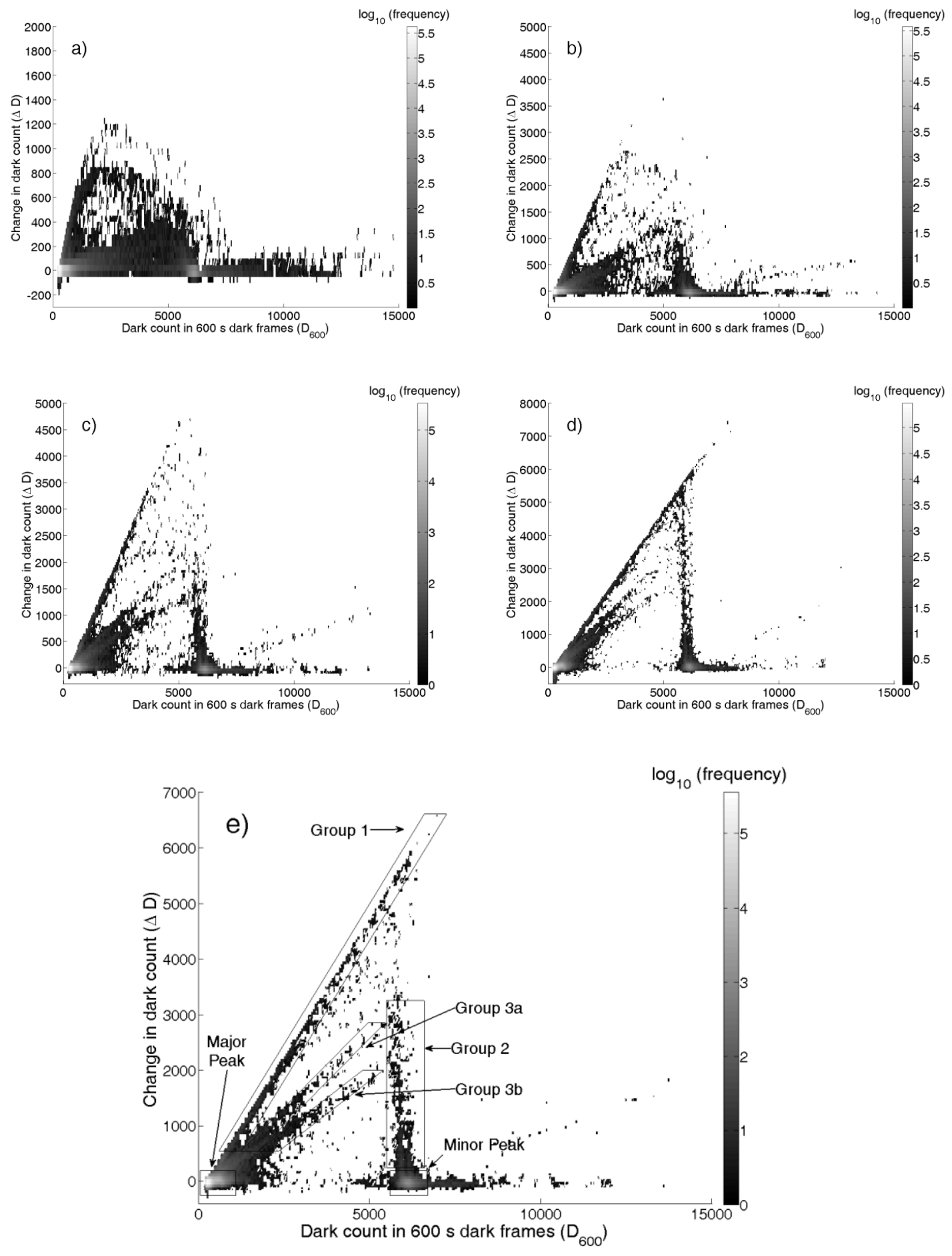

Fig. 2.3: Distribution of the change in dark count, $\Delta D_{\text {light }}$, at $288 \mathrm{~K}$ due to illumination for the KAF-1602E for three different light levels. The average initial counts due to the light exposure were approximately: a) 
2,500 counts, b) 8,500 counts, c) 16,000 counts, d) 37,000 , and e) 26,000 counts.

The greatest change in dark counts is exhibited by the pixels in Group 1. The change in dark count for those pixels is almost as high as their initial dark count. Hence, these pixels almost stopped generating dark current after being illuminated. One such pixel is Pixel 1 in Fig. 2.2. Under "normal" conditions, without the presence of light, its dark count at $288 \mathrm{~K}$ and $600 \mathrm{~s}$ exposure time is 1,139 counts. From Fig. 2.2 one observes that for an initial illumination level of roughly 8,000 counts or more, the decrease is almost 1,000 counts. This corresponds to only about $14 \%$ of the dark count without the presence of light (see Table 2.2).

In Fig. 2.3 a straight line can be drawn to fit all the pixels in Group 1. The slope of the straight line fit is larger than 0.9 for all five illumination levels depicted. As a result, pixels in Group 1 have a decrease in dark count of about $90 \%$ (e.g. $85.6 \%$ for Pixel 1 in Fig. 2.2) of the dark count. Hence, those pixels show almost no dark count when flashed with an initial illumination level of 8,000 counts or more (see Table 2.2).

Both Groups 3a and 3b show a decrease in dark level under illumination. Pixels 3a and $3 \mathrm{~b}$ are representative of these groups. From Fig. 2.2, one can see that the dark level decreases with increasing illumination over the whole range of illumination levels. The percentage decrease of the dark count is smaller than for Group 1. The decrease of the dark level for those pixels for the different illumination levels can again be found from the slope of a linear fit through the pixels of these groups. Pixels in Group 3a have a decrease of about $25 \%$ at 8,600 counts illumination (Fig. 2.3b), 
$40 \%$ at 16,000 counts illumination (Fig. 2.3c), and $60 \%$ at 26,000 counts illumination (Fig. 2.3e). Hence, the dark current decrease is from $75 \%$ of the normal level at 8,600 count illumination to $40 \%$ of the normal level at 26,000 count illumination. Group $3 b$ has the same characteristics as Group 3a, with the percentage decrease being slightly smaller. At 8,600 counts illumination the dark count is decreased by $15 \%$, at 16,000 counts illumination it is decreased by $25 \%$, and at 26,000 counts illumination it is decreased by $50 \%$.

Finally, Group 2 is formed by pixels that have the same normal dark count as the pixels grouped in the minor peak. However, under illumination their dark current decreases. As for Groups $3 \mathrm{a}$ and $3 \mathrm{~b}$, the dark count decreases with increasing illumination in Group 2. Pixel 2 in Fig. 2.2 belongs to this group. The percentage change in dark count for all pixels in Fig. 2.2 are presented in Table 2.2. 


\begin{tabular}{|l|c|c|c|c|c|}
\hline Group Name & \# Pixels & $\mathbf{D}_{600}$ in counts & $\begin{array}{c}\Delta \boldsymbol{D}_{\text {light }} \text { at } \\
\mathbf{8 , 5 0 0} \text { counts }\end{array}$ & $\begin{array}{c}\Delta \boldsymbol{D}_{\text {light }} \text { at } \\
\mathbf{1 6 , 0 0 0} \\
\text { counts }\end{array}$ & $\begin{array}{c}\Delta \boldsymbol{D}_{\text {light }} \text { at } \\
\mathbf{2 6 , 0 0 0} \\
\text { counts }\end{array}$ \\
\hline Major Peak & $1,519,003$ & 50 to 1,100 & -250 to 200 & -250 to 200 & -250 to 200 \\
\hline Minor Peak & 16,628 & 5,800 to 6,200 & -250 to 200 & -250 to 200 & -250 to 200 \\
\hline Group 1 & 3,638 & 1,000 to 6,000 & $90 \%$ of $D_{600}$ & $90 \%$ of $\mathrm{D}_{600}$ & $90 \%$ of $\mathrm{D}_{600}$ \\
\hline Group 2 & 757 & 5,500 to 6,600 & 250 to 3,250 & 250 to 3,250 & 250 to 3,250 \\
\hline Group 3a & 1,347 & 1,750 to 5,450 & $25 \%$ of $D_{600}$ & $40 \%$ of $D_{600}$ & $60 \%$ of $D_{600}$ \\
\hline Group 3b & 1,669 & 2,350 to 5,400 & $15 \%$ of $D_{600}$ & $25 \%$ of $D_{600}$ & $50 \%$ of $D_{600}$ \\
\hline Other & 17,558 & Varies & Varies & Varies & Varies \\
\hline
\end{tabular}

Table 2.3. Change in dark count for the different groups under different illumination levels for the KAF-1602E. The number of pixels in each group was calculated using the constraints shown in Fig. 2.3 at 26,000 counts.

A summary of the number of pixels in the individual groups and their change in dark count under different illumination levels can be seen in Table 2.3. Of the pixels outside these groups, 3,467 pixels have no change in dark count under illumination. Furthermore, there are a small number of pixels that have a dark count larger than in the minor peak and a slight decrease with illumination. We found that there are a significantly larger number of those pixels in the KAF-0400 chip and we will look at them in more detail in the next section. There are also a number of pixels that show the opposite effect and have an increase in dark current with illumination. Most of these lie below the Main Peak and we found that these pixels consistently have a very hot neighboring pixel. These pixels will be discussed in more detail below. 


\subsubsection{KAF-0400}

The second CCD investigated in this study was the Kodak KAF-0400 housed in the Meade Pictor 416XT. For the Pictor 416XT camera, data at $278 \mathrm{~K}$ was taken. The experimental setup and the analysis were done in the same way as for the SBIG camera.

Figure 2.4 shows the distribution for the change in dark count for three different illumination levels. The images were flashed with light that caused an average count of approximately 8,500 (upper left panel), 16,000 (upper right panel) and 26,000 counts (lower panel).

One finds many similarities and some differences in the characteristic of the graphs for the two chips. For the KAF-0400, instead of the two peaks, one finds another minor peak. The majority of pixels are regular pixels with very little dark current located in the major peak. Their average dark count at $278 \mathrm{~K}$ and $600 \mathrm{~s}$ exposure time is about 500 counts. There are two groups of pixels with a dark count of approximately 5,700 counts (Minor Peak 1) and 10,900 counts (Minor Peak 2), respectively. It is interesting to notice that pixels in all three peaks have a dark count of about 250 counts more when illuminated to light. The normal dark count of the pixels in Minor Peak 2 is about twice the dark count of the Minor Peak 1 pixels, making it likely that the same impurity occurs twice within that group. 
Group 1a for the KAF-0400 is analogous to Group 1 for the KAF-1602E. A straight line drawn through the pixels of Group 1a has a slope of approximately 1. Hence, pixels in this group generate virtually no dark count after illumination. Of course, to this one needs to add the global positive offset of about 250 counts. One can also see a number of pixels emerge from the first minor peak with a similar slope to the pixels in Group 1a. Because of this similarity we will call those pixels Group 1b. There are 1,376 pixels in Group 1b, less than $10 \%$ of the number of pixels found in Group 1a (see Table 2.3). This is still a sizeable number, and one generally notices that there are a much larger number of pixels outside the Major Peak and Minor Peak groups for the KAF-1602E. Only about $80 \%$ of the pixels are in the Major Peak for the KAF-1602E imager, whereas for the KAF- 0400 imager about $97 \%$ are in the Major Peak. One can explain pixels in Group $1 \mathrm{~b}$ by assuming the presence of a Minor Peak 1 plus Group 1a type impurity in the same pixel.

Using the same reasoning for Group 2 one can conclude that Group $2 b$ pixels contain a Minor Peak 1 plus a Group 2a type impurity. Again the number of pixels in Groups $2 \mathrm{a}$ and $2 \mathrm{~b}$ is much larger than the number of pixels in Group 2 of the KAF-1602E. Only $0.05 \%$ of all pixels on the chip are in Group 2, while the percentage is much larger for the corresponding Group $2 \mathrm{a}$ and Group $2 \mathrm{~b}$ with $1.8 \%$ and $0.3 \%$, respectively (see Table 2.3 and Table 2.4). Groups $3 \mathrm{a}$ and $3 \mathrm{~b}$ for the KAF-1602E do not a have an equivalent in the KAF-0400. Instead, there are a larger number of pixels (Group 3 with 1,751 pixels) with a normal dark count larger than the first Minor Peak and a significant loss in dark count. The slope of a linear fit through Group 3 pixels in 
Fig. 2.4 at 42,100 counts is given by about 0.3 . It appears likely that those pixels are the result of a Minor Peak 1 plus another secondary impurity. One can further notice from Fig. 2.3 that the KAF-1602E imager seems to contain a similar type of pixel as well. However, just as for the rest of the chip, the number of pixels with impurities was smaller and we did not assign them to a separate group. Groups $2 \mathrm{~b}$ and 3 overlap and we counted 427 pixels twice as being both in Group $2 \mathrm{~b}$ and in Group 3. 9,931 pixels are not in any of the groups, with 5,045 of those at the baseline with an increase in the dark count of approximately 250 counts.

\begin{tabular}{|c|c|c|c|c|}
\hline Group Name & \# Pixels & $\mathbf{D}_{600}$ in counts & $\begin{array}{c}\Delta \boldsymbol{D}_{\text {light }} \text { at } \\
\mathbf{2 5 , 5 0 0} \text { counts }\end{array}$ & $\begin{array}{c}\Delta \boldsymbol{D}_{\text {light }} \text { at } \\
\mathbf{4 2 , 1 0 0} \text { counts }\end{array}$ \\
\hline Major Peak & 322,051 & 50 to 1600 & -700 to 100 & -700 to 100 \\
\hline Minor Peak 1 & 30,818 & 5,000 to 6,600 & -600 to 100 & -600 to 100 \\
\hline Minor Peak 2 & 1,550 & 10,100 to 11,800 & -450 to 100 & -450 to 100 \\
\hline Group 1a & 16,332 & 400 to 7,400 & $1 \times \mathrm{D}_{600}-$ Off & $1 \times \mathrm{D}_{600}-\mathrm{Off}$ \\
\hline Group 1b & 1,376 & 6,200 to 11,700 & $1 \times\left(\mathrm{D}_{600}-\mathrm{D}_{\mathrm{MP} 1}\right)-$ & $1 \times\left(\mathrm{D}_{600}-\mathrm{D}_{\mathrm{MP} 1}\right)-$ \\
Off
\end{tabular}

Table 2.4: Change in dark count for the different groups under different illumination levels for the KAF-0400. The parameter, Off $=250$ counts, represents the dark count decrease of a regular pixel under illumination. The dark count of a pixel in the first minor peak is given approximately as $D_{M P I}=5,700$ counts. The number of pixels in each group was calculated using the constraints shown in Fig. 2.4 at 42,100 counts. 

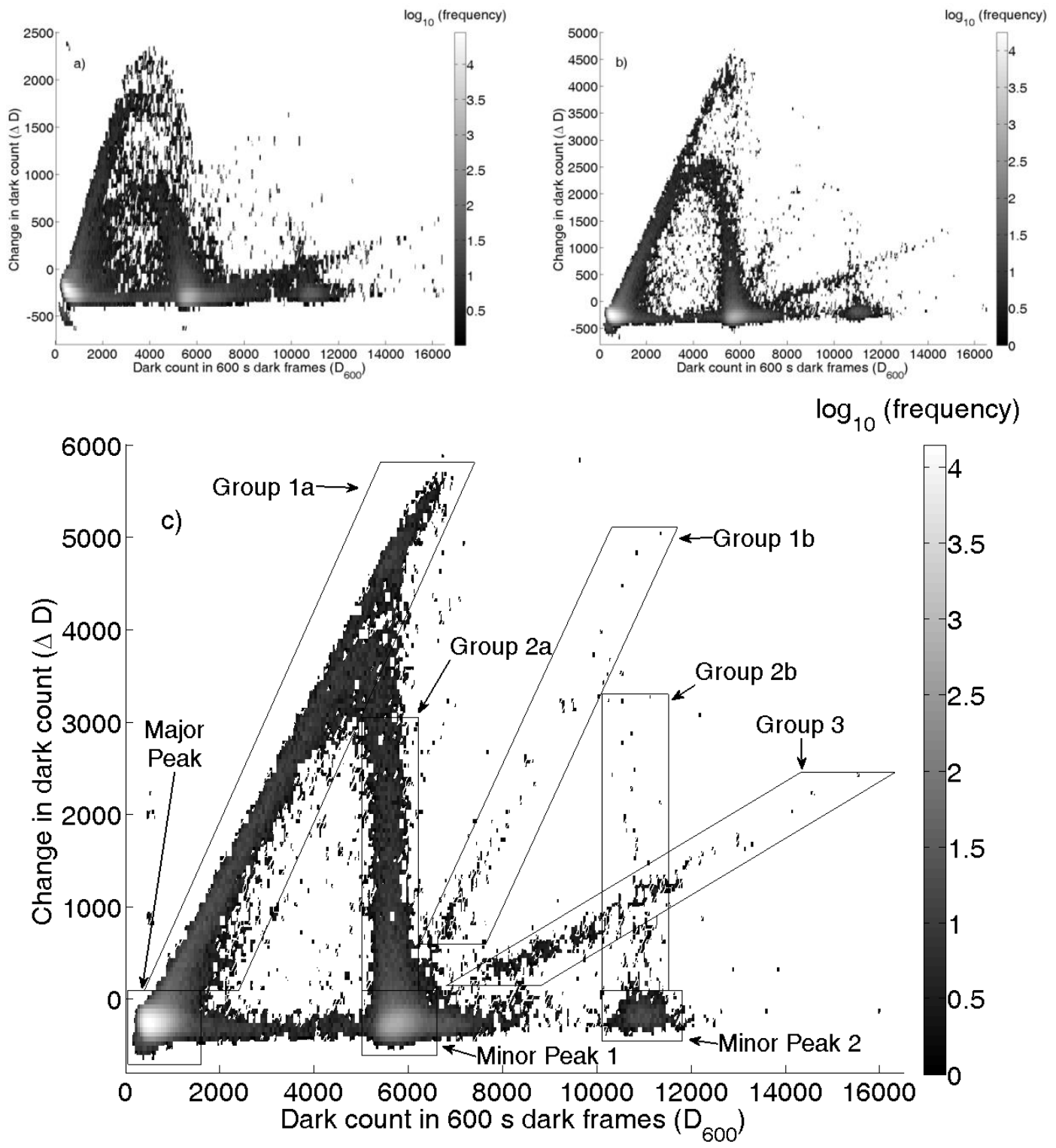

Fig. 2.4: Distribution of the change in dark count at $278 \mathrm{~K}$ due to illumination for the KAF-0400 for three different light levels. The average initial counts due to the light exposure were approximately: a) 12,500 counts, b) 25,500 counts, and c) 42,100 counts. 


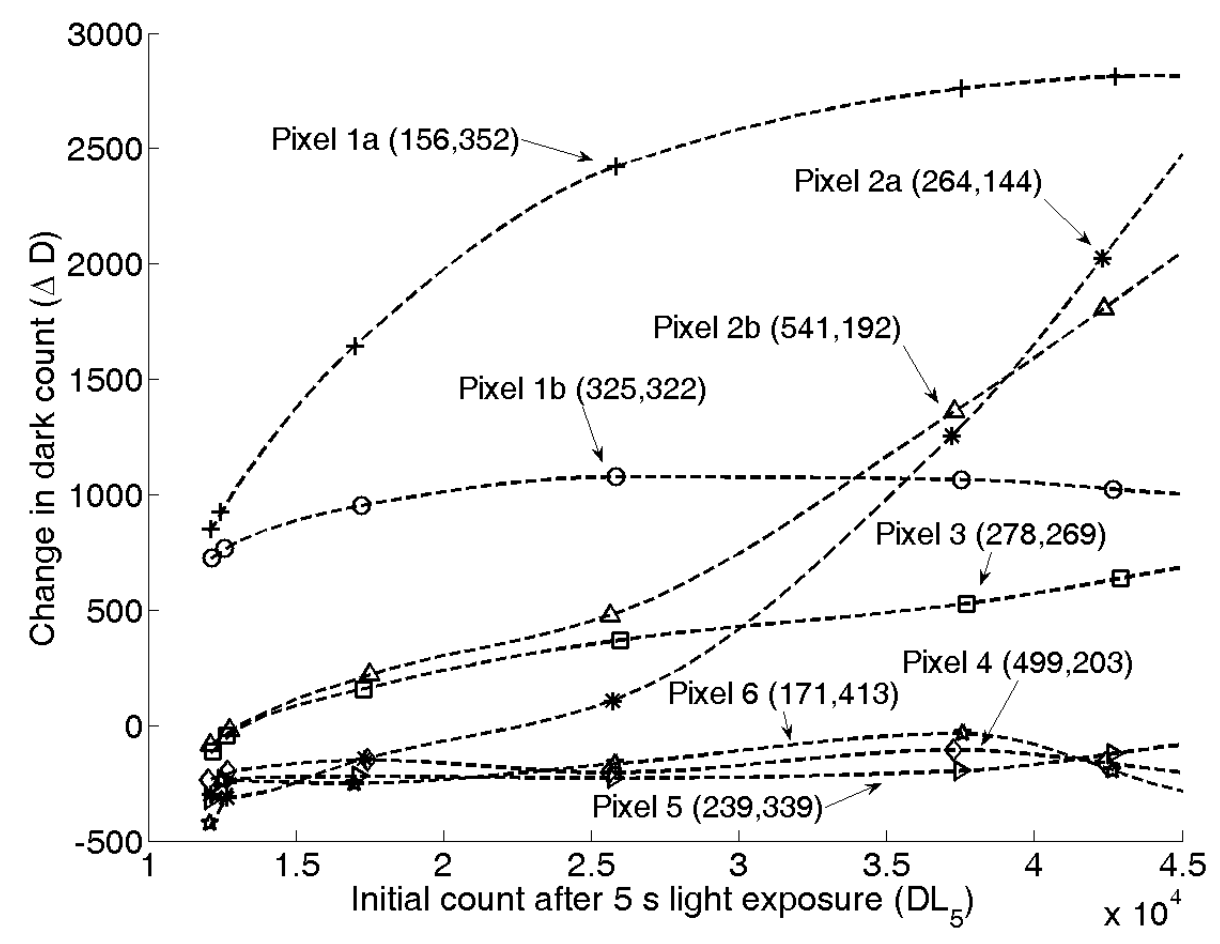

Fig. 2.5: Loss in dark count vs. light level for 8 pixels for the KAF-0400. The numbers in the brackets are the $\mathrm{x}$ and $\mathrm{y}$ coordinates of the pixel.

As can be seen from Fig. 2.5, the pixels on the KAF-0400 imager respond to increasing illumination in a similar manner as do the corresponding groups on the KAF-1602E sensor shown in Fig. 2.2. The pixels lying in the peaks, in this case Pixels 4, 5, and 6 lie in the Main Peak, Minor Peak 1, and Minor Peak 2 respectively, do not show a significant change with changing illumination level. Pixels 1a, 1b, 2a, $2 \mathrm{~b}$, and 3 all show a decrease in dark count with increasing levels of illumination, and all lie within their correspondingly named region in Fig. 2.4c. For Pixels $1 \mathrm{a}$ and $1 \mathrm{~b}$ the amount of dark current levels off for higher illumination levels. The decrease in dark count is roughly constant for Pixel $1 \mathrm{~b}$ after about 20,000 counts and is roughly constant for Pixel 1a after 35,000 counts. 


\subsection{Influence of saturated neighboring pixels}

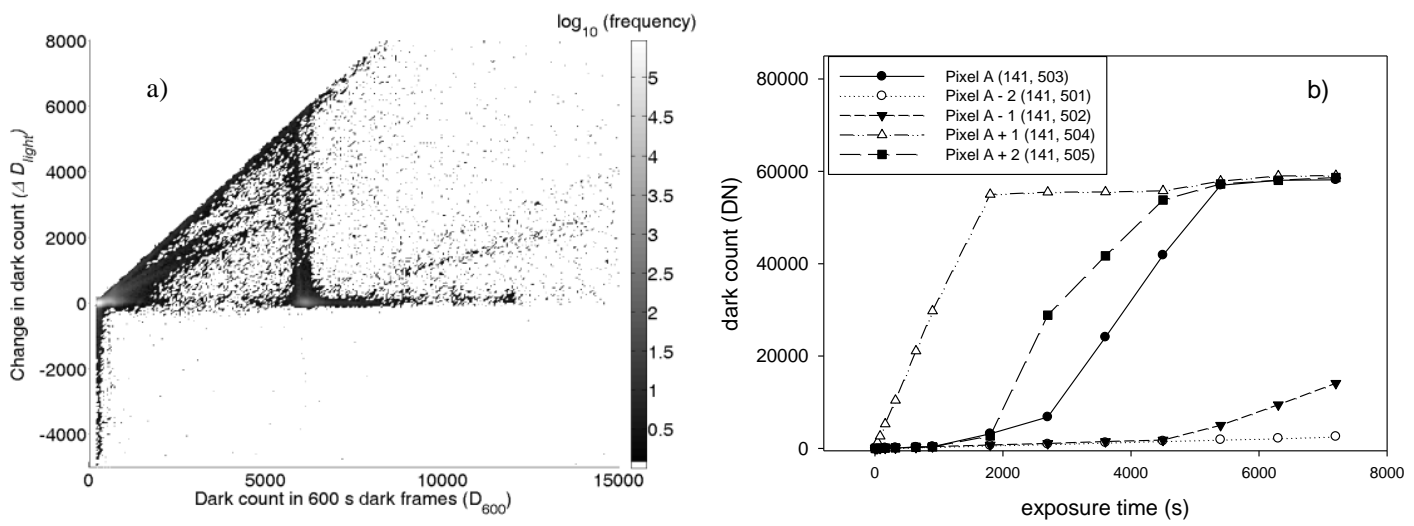

Fig. 2.6: a) Distribution of the change in dark count at $288 \mathrm{~K}$ due to illumination for the KAF-1602E for 37,500 counts. b) Exposure time dependence of a pixel showing negative change in dark count and its neighboring pixels.

A grouping of pixels can be seen in Fig. 2.3 lying below the Major Peak. A similar grouping is seen for the KAF-1602E, however there are far more pixels of this type in the KAF-0400. Shown in Fig. 2.6a is the same graph shown in Fig. 2.3d, with the change in dark count extended down to -5000 counts showing the large number of pixels with a negative change in dark count. These pixels actually show an increase in dark current with exposure to light. This effect can be explained by the saturation of neighboring pixels. Shown in Fig. 2.6b is the dependence of dark current on exposure time of one of the pixels (Pixel A) seen in that grouping as well as its four nearest neighbors in the same column. One of its direct neighbors, the pixel above it on the column (Pixel $A+1)$, is seen to saturate at about the same point that the pixel below, Pixel A, and the pixel above, Pixel A+2, begins to show an increase in dark counts. This can be explained by the saturated pixel $(A+1)$ leaking some of its stored charge 
into neighboring pixels once its well is filled. Examining other pixels in the same grouping also showed a neighboring pixel that would saturate before a change in the rate of dark current collection was seen in the original pixel. This effect is actually seen again in Fig. 2.6b when the pixel directly below Pixel A, Pixel A-1, begins to show an increase in the rate of dark current collection after Pixel A itself becomes saturated. This phenomenon is usually referred to as blooming and manifests itself in images as lines of saturated pixels.

\subsection{Discussion}

We found that results are independent of the wavelength, $\lambda$, of the illuminating light and therefore independent of the location where photoelectrons are generated. The data for a red LED with $\lambda=650 \mathrm{~nm}$ and a penetration depth in silicon of $3 \mu \mathrm{m}$ yielded the same characteristics as a blue LED with $\lambda=430 \mathrm{~nm}$ and a penetration depth of only $300 \mathrm{~nm}$.

The distributions for the same light level at different temperatures were measured and the same characteristics were found at those temperatures as well. It was further noted that the pixels of all groups were found to be uniformly distributed over the whole area of the chip.

Most pixels for the KAF-1602E sensor fall in the two peaks and do not show a significant change. On the other hand, for larger intensities a majority of pixels for the KAF-0400 show an actual increase in dark count. Hence, a regular dark frame would 
predict a dark count which is too small. Our data indicate that this is a global phenomenon across the whole chip, resulting in a constant offset. What makes an accurate dark count correction more problematic is the fact that some pixels will show a decrease in dark count. Under large enough illumination, pixels in Group 1 almost stop generating dark electrons. Of course, in an actual exposure the photo-excitation of electrons due to the incoming light does not happen all at the beginning of the exposure. Assuming a constant intensity of light, a pixel in Group 1 might generate dark current at a normal rate at the beginning of the exposure and then transforms to a non-dark count generating state toward the end of the exposure. Our analysis can help to predict how much dark count is generated during different stages of the exposure. This, however, assumes that the light intensity is constant and does not vary with time. If one does not have sufficient knowledge of changes in the light intensity during the exposure, an accurate prediction of the dark count of pixels in Group 1 is virtually impossible.

The difficulties in correcting dark current accurately for pixels in Groups 2 and 3 are similar to those for pixels in Group 1 . There are roughly 21,000 pixels, or $1.4 \%$ of the total pixels, for the KAF-1602E sensor ( $T=288 \mathrm{~K}, 600 \mathrm{~s}$ exposure time) where the dark current differs by more than 200 counts for an exposure with an initial illumination level of 26,000 counts. The same pixels would show a different dark count to a varying degree under all illumination conditions. Ignoring the global increase of dark current under illumination, there are roughly 28,000 pixels, or $7.1 \%$ of the total pixels, 
in Groups 1, 2, and 3 that have a dark count that is strongly illumination level dependent for the KAF-0400 sensor.

Finally, since dark current is strongly linked to impurities in the CCD our results can lead to a new understanding of the nature of those impurities. Impurities are generally characterized by the dark current they produce at a particular exposure time and temperature. By varying the temperature one can furthermore find the activation energy of a particular impurity. The activation energy can, for example, be used to distinguish between diffusion current generated in the field-free region or depletion current from within the potential well [23]. But the nature of impurities also differs by their behavior under illumination. A pixel in the Minor Peak and a Group 2 pixel show the same normal dark count. They would be characterized as being hot and containing one particular impurity. However, our data clearly shows that while they may have the same impurity, the nature of the defect in these pixels differs as evidenced by their response to illumination.

\subsection{Summary}

Dark current is generally assumed to be independent of the light level of exposure. Differences between the actual number of dark electrons in the real exposure and a dark frame are generally assumed to be due to statistical noise. However, our study shows clearly that certain pixels show a systematic difference in their dark count under illumination. 
The change in dark count $\Delta D_{\text {light }}$ in $600 \mathrm{~s}$ cannot assume any value - we found that only specific areas in the $\Delta D_{\text {light }}$ vs. $D_{600}$ plane were observed. We were able to group the behavior of pixels according to this discrete change. Pixels in one group may contain one specific type of impurity.

We hope this study will help contribute to a better understanding of the nature of dark current and an even further improvement of CCD image quality. More immediately the results help to accurately correct for dark current and in the computation of dark frames. 


\section{CHAPTER 3 - NONLINEAR EXPOSURE TIME RESPONSE OF CCD PIXELS}

\subsection{Introduction}

In this section we present a systematic study on the rate of dark current generation of two scientific CCD imagers. The dark current in both imagers was measured for exposure times from $5 \mathrm{~s}$ to $7200 \mathrm{~s}$ at a constant temperature. As one would expect, the majority of pixels show a linear increase in dark count with exposure time. However, we found distinct groups of pixels that show a nonlinear dark current dependence versus exposure time well below saturation. Since the dark count is often assumed to scale linearly with exposure time, these pixels can pose a problem during dark current correction. We also discuss what could cause some pixels to produce a dark count which is linear versus exposure time whereas others do not. Much of the material presented in this chapter has been published in [26].

At the heart of a digital imaging system often sits a silicon CCD sensor. In the absence of light, electrons can be thermally excited from the valence to the conduction band of the silicon. The temperature dependence of dark current has been thoroughly studied [6], [24], [8], [9]. The dark count signal depends approximately exponentially on the temperature [23]. Depending on the chip design, the surface, depletion, and diffusion dark currents can contribute in varying amounts to the total dark current. Their contributions can be distinguished, for example, by their characteristic activation energy. The characteristic activation energy is half the bandgap of silicon for the surface 
generated dark current and the dark current generated in the depletion region, and is equal to the bandgap of silicon for the diffusion related dark current [18]-[22]. Some complications to this characterization will be explored in Chapter 7. Not all pixels produce the same amount of dark current. So called hot pixels, pixels with an unusual high dark count, are distributed seemingly randomly across a CCD chip. The high dark count of the hot pixels is caused by impurities in the silicon. Such impurities can be lattice defects, or foreign atoms (e.g. Fe, Ni, and Au) [6], [24], [8], [9].

In order to minimize dark current, scientific imagers are often cooled. One can correct for dark current by subtracting an image, taken with a closed shutter, from the exposed image. To get an accurate reflection of the dark current one needs to take the dark image under the same condition as the exposed image. The generation of dark current is a statistical process described by the Poisson distribution. Taking multiple dark frames under the same condition, and averaging the frames to create a master frame, improves the signal to noise ratio. Obtaining such a master dark frame can be time consuming but often leads to a significantly improved noise correction. Master dark frames can, in principle, be reused if the temperature and exposure time are unchanged. We have shown that dark frames can also be computed for any temperature after an initial calibration of the imager [18].

It is generally assumed that the dark count increases linearly with exposure time. In principle this allows dark frames to be scaled to different exposure times. To do this, the bias offset, Bias, is subtracted and the thermal signal is obtained. The bias is a 
consistent offset introduced when the detector is read out that varies from pixel to pixel in a repeatable fashion. To obtain a dark frame for a different exposure time, the thermal signal is scaled by a factor corresponding to the desired exposure time. The bias offset can then be added back to the scaled thermal signal to obtain the final scaled dark frame for the new exposure time. For example a $60 \mathrm{~s}$ dark frame, $D_{60}$, can be obtained from a 600 s frame, $D_{600}$, by calculating:

$$
D_{60}=1 / 10 *\left(D_{600}-\text { Bias }\right)+\text { Bias }
$$

The advantage of a scaled dark frame is that a longer exposure frame provides a large signal to noise ratio; the scaling of that frame creates a short exposure frame which would have a larger signal to noise ratio than a frame taken at that exposure time. Fig. 3.1 shows the results obtained for scaled dark frames for two different cameras: the SBIG ST-8XE with a KAF-1602E CCD sensor and the Meade Pictor 416XT with a Kodak KAF-0400 CCD chip. The histograms of the count difference between a single $60 \mathrm{~s}$ dark frame and a master dark frame obtained from the average of ten $60 \mathrm{~s}$ exposures are shown in Fig. 3.1b. Ideally the distribution would show a single peak with all pixels having zero counts. Readout noise as well as dark current shot noise cause a widening of the distribution. The width of the distribution, as given by its standard deviation, is equal to 26.3 counts. In Fig. 3.1a, a $600 \mathrm{~s}$ frame is scaled to $60 \mathrm{~s}$ and subtracted from an individual $60 \mathrm{~s}$ frame. It took approximately the same camera time to obtain the scaled $60 \mathrm{~s}$ frame as it took to get the $60 \mathrm{~s}$ master frame. However, the scaled frame only requires one readout and should therefore have less readout noise. The histogram in Fig. 3.1a exhibits a long tail with many pixels having larger values for the individual frame than for the scaled frame. The resulting standard deviation has 
increased to 90.9 counts. The results for the Meade camera are similar and exhibit a poorer correction with the scaled frame (Fig. 3.1c) than for the master frame (Fig. 3.1d). Subtraction using the scaled frame shows a distinct second peak at approximately minus 350 counts in the resulting histogram. Hence, a group of pixels show a larger count when scaled from a long exposure frame.

For both cameras the result of the scaled dark frames is not quite as good as one would expect assuming a linear time dependence of the dark count. We found that the explanation of the poor correction of the scaled frame requires considering nonlinearities of the dark current versus exposure time. Such nonlinearities are well known when the count reaches values close to the full well of a pixel. However, nonlinearities at lower signals have not been studied in detail.

In this work we characterize the dark current of the two aforementioned sensors for different exposure times. We find that certain pixels exhibit a predictable nonlinear behavior. Furthermore, the nonlinear behavior of pixels can be categorized in groups with similar characteristics. 

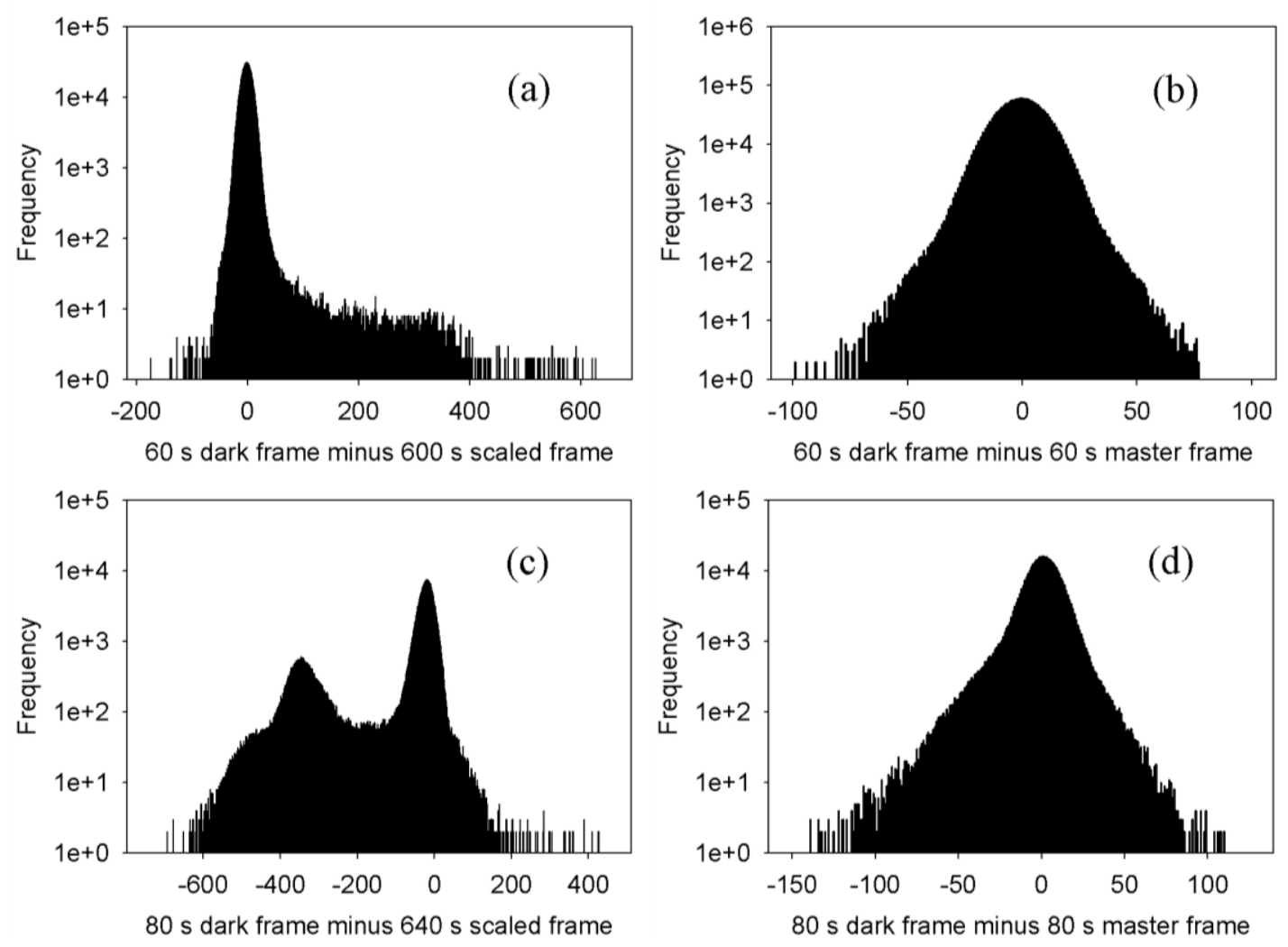

Fig. 3.1. Difference of an individual dark frame and a master frame, and the individual dark frame and a scaled dark frame. (a) Single $60 \mathrm{~s}$ frame minus an individual $600 \mathrm{~s}$ frame scaled to $60 \mathrm{~s}$ for the KAF-1602E at T=288K.(b) Single $60 \mathrm{~s}$ frame minus the average of ten $60 \mathrm{~s}$ frames for the SBIG imager at $\mathrm{T}=288 \mathrm{~K}$. (c) Single $80 \mathrm{~s}$ frame minus a single $640 \mathrm{~s}$ frame scaled to $80 \mathrm{~s}$ for the Meade imager at $\mathrm{T}=278 \mathrm{~K}$. (d) Single $80 \mathrm{~s}$ frame minus the average of eight $80 \mathrm{~s}$ frames for the KAF-0400 at $\mathrm{T}=278 \mathrm{~K}$.

\subsection{Experimental procedures and results}

\subsubsection{KAF-1602E}

We obtained dark frames at $288 \mathrm{~K}$ for exposure times from 5 to $7200 \mathrm{~s}$. For exposure times smaller than $640 \mathrm{~s}$ the median pixel value of three frames was used. One frame was taken for exposure times of $900 \mathrm{~s}$ and above. 

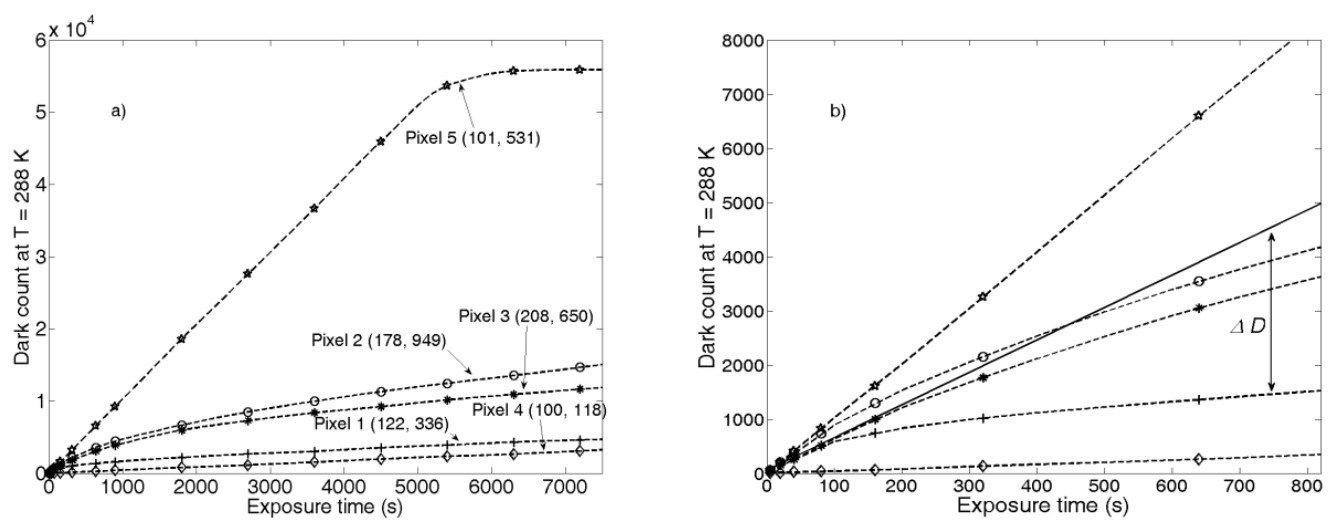

Fig. 3.2: Dark count at $288 \mathrm{~K}$ as a function of exposure time for 5 pixels for the KAF-1602E. (a) Exposure times up to 7200 s. (b) Enlargement with exposure times up to $800 \mathrm{~s}$ and linear fit to Pixel 1 (same pixels shown as in (a)).

Figure 3.2a shows the dark current of 5 different pixels. The majority of pixels on the chip behave similarly to Pixel 4 . The dark count is low and seems to increase linearly with exposure time. The other pixels show a larger dark count and would be considered to be hot pixels. The pixel with the highest dark count is Pixel 5. Its dark count increases approximately linearly up to 50,000 counts. This is actually slightly larger than the saturation count as listed by the manufacturer. Once a pixel has reached saturation, electrons start to spill into neighboring pixels. Therefore, the dark count of a saturated pixel, as well as its neighbors, has to be considered with care. For exposure times shorter than about $100 \mathrm{~s}$, the dark count of Pixel 2 is similar to the dark count of Pixel 5 (see Fig. 3.2b). However, whereas the dark signal increases linearly up to saturation for Pixel 5, Pixel 2 levels off much earlier. Pixel 3, while less hot than Pixel 2, also shows a decreasing slope versus exposure time. 
This effect is even more dramatic for Pixel 1 as the dark count up to about $50 \mathrm{~s}$ is as high as the dark count of Pixel 5. For example at $40 \mathrm{~s}$ its dark count is 79 percent of the dark count of Pixel 5. However, at 7,200 s Pixel 1 has only 8 percent the dark count of Pixel 5. At 7200 s, Pixel 1 generates dark current at the same rate as a regular pixel, as represented by Pixel 4. On the other hand, at low exposure times the pixel behaves more like a hot pixel. Using the dark count at short exposure times might therefore not always be a good measure of how a pixel behaves at long exposure times.

To get a global overview of the linearity and nonlinearity of all pixels on the chip a linear fit of the type;

$$
D_{\text {fit }}^{i j}=b^{i j} t
$$

was applied to dark frames from $5 \mathrm{~s}$ to $60 \mathrm{~s}$ (see solid line in Fig. 3.2b as the linear fit for Pixel 1). Hence, the parameter $b^{i j}$ represents the dark current in counts per second over this time interval for the ijth pixel. If it is assumed that the dark current is linear, the dark count can be calculated at any exposure time, $t_{0}$, as $D_{f i t}\left(t_{0}\right)=b t_{0}$. One can conclude from Fig. 3.2 that this linear scaling to a different exposure time works well for pixels such as Pixel 4 and Pixel 5. However, for pixels such as Pixels 1, 2, and 3, which show a nonlinear dark current behavior, one predicts an incorrect value for the dark count. As seen in Fig. 3.2b, pixels that show a decreasing slope in the dark count versus exposure time plot will have an actual dark count that is lower than predicted by the fit. To evaluate the difference between the dark count obtained from the linear fit, $D_{f i t}$, and the measured dark count, $D_{\text {measured }}$, we define:

$$
\Delta D=D_{\text {fit }}\left(t_{0}\right)-D_{\text {measured }}\left(t_{0}\right)=b\left(t_{0}\right)-D_{\text {measured }}\left(t_{0}\right)
$$


According to Eq (3.3) for Pixel 1 evaluated at $t_{0}=160 \mathrm{~s}, \Delta D$ is 295 counts. For a longer exposure time of $t_{0}=640 \mathrm{~s}$, this difference has increased to 2778 counts.
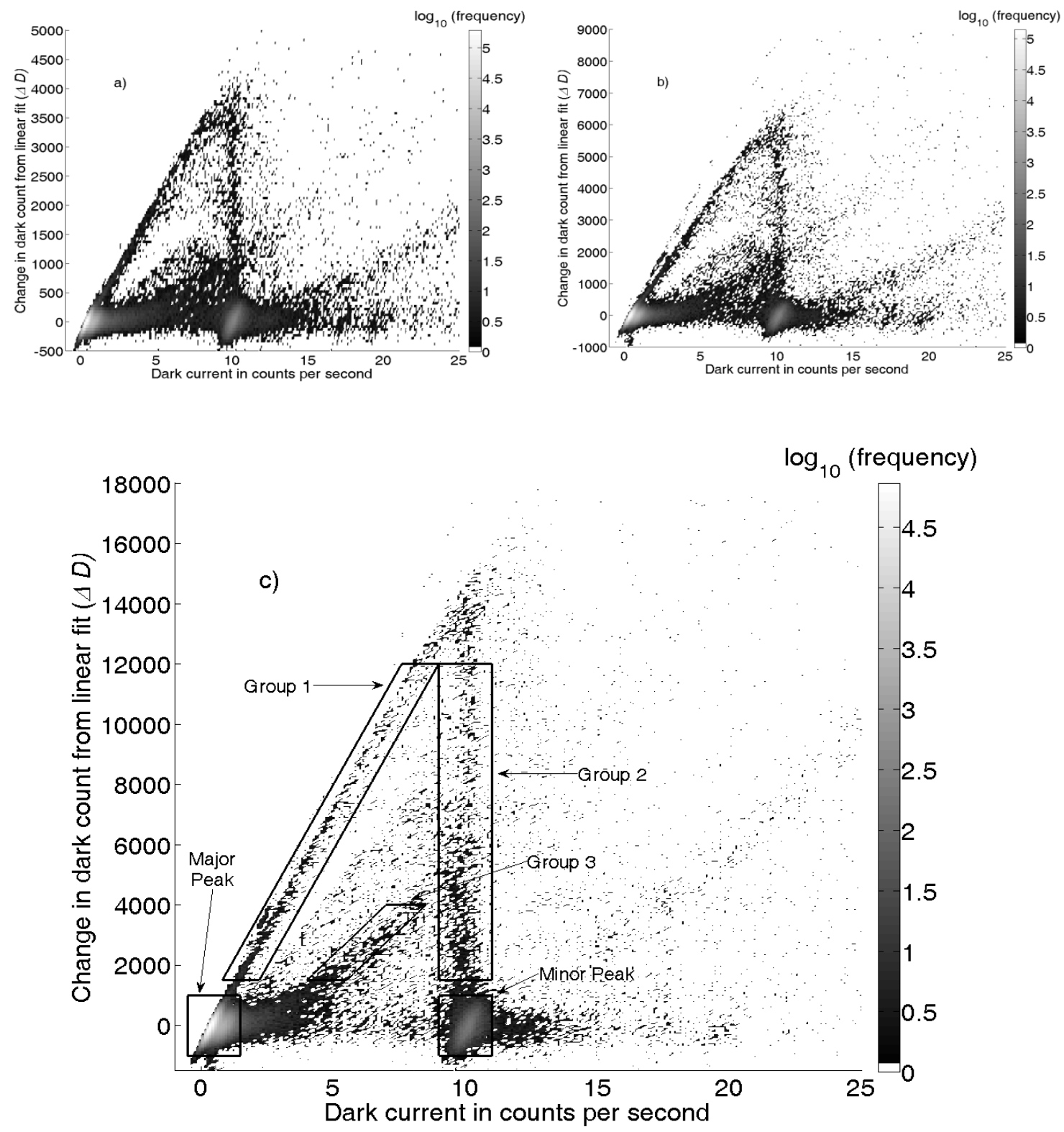

Fig. 3.3: Dark current from linear fit versus the change in dark count, $\Delta D$, for the KAF-1602E at $288 \mathrm{~K}$ evaluated at (a) $640 \mathrm{~s}$, (b) $900 \mathrm{~s}$, (c) $1800 \mathrm{~s}$. 
Fig. 3.3 shows the distribution of the change in dark count, $\Delta D$, at $t_{0}=640 \mathrm{~s}$ (Fig. 3.3a), $t_{0}=900 \mathrm{~s}$ (Fig. 3.3b), and $t_{0}=1800 \mathrm{~s}$ (Fig. 3.3c) versus the dark current which is given as the parameter $b$ in Eq. (3.2) for exposure times from 5 to $60 \mathrm{~s}$. For the figure the change in dark count vs. dark current plane is divided into areas of size 0.1 counts/s (dark current as given by the parameter $b$ in Eq. 3.2) by 50 counts in the $y$-direction $(\Delta D)$. The number of pixels in each area, the frequency, is then plotted. By far, the largest number of pixels is located in the Major Peak where approximately 1.52 million pixels (or 97 $\%)$ lie within the rectangle indicated in Fig. 3.3c. Another 17,585 pixels are in the Minor Peak with a dark current of approximately 10 counts/s and $\Delta D \approx 0$. Pixel 4 is an example of a pixel in the Major Peak while Pixel 5 is a Minor Peak pixel. Pixel 2, a typical Group 2 pixel, behaves like a Minor Peak pixel with a dark current of approximately 10 counts/s based on the linear fit. However, as one can see in Figs. 3.2 and 3.3, Group 2 pixels are nonlinear and have a lower dark count for larger exposure times. There are a number of pixels where the change in dark count, $\Delta D$, is larger than the actual dark count. Pixels defined by Group 1 have a change in dark count which is approximately 5 times the dark count value at 1800 s. For example Pixel 1 has a dark count of 2,265 counts at $1800 \mathrm{~s}$, a predicted dark count based on a linear fit of 10,864 counts and hence a change in dark count of 8,599 counts. About 1,500 pixels are within the parallelogram identifying Group 1. Group 3 pixels, as represented by Pixel 3 in Fig. 3.2, also show a change in dark count, however their nonlinear behavior is smaller in magnitude. One also notices from Fig. 3.3 that a smaller number of pixels behave similarly to Group 3 pixels, but emerge from the Minor Peak rather than the Major Peak. Another distinct group of pixels could be identified in between Group 1 and 
Group 3. For clarity sake, we refrained from giving those pixels their own group designation. Other major distributions of pixels in Fig. 3.3 are pixels which are not located in the two peaks but, similar to the pixels in the peaks, have no significant change in dark count (i.e. $\Delta D \approx 0$ ). Finally, there are a number of pixels not shown in the graph that have a small dark current like Major Peak pixels and a negative change in dark count. We found that these pixels are located next to a hot pixel that saturated at longer exposure times, spilling electrons into neighboring pixels.

\subsubsection{KAF-0400}

From Fig. 3.1 we found that the KAF-0400 sensor in the Pictor 416XT camera, like the KAF-1602E sensor in the SBIG camera, shows a non-perfect correlation between a single dark frame and a scaled dark frame. Graphs similar to Fig. 3.3 were generated in order to find out if the sensor in the Meade camera also shows nonlinear dark current behavior that can be separated in distinct groups. Fig. 3.4 shows graphs with the dark current on the x-axis calculated from exposure times of 40 to $320 \mathrm{~s}$ at $T=278 \mathrm{~K}$. A linear fit of the dark count to those exposure times was applied and extrapolated to $640 \mathrm{~s}$ (Fig. 3.4a), $900 \mathrm{~s}$ (Fig. 3.4b), and $1800 \mathrm{~s}$ (Fig. 3.4c). As in the previous section, the change in dark count, $\Delta D$, is then calculated for each pixel and displayed on the y-axis. The shades of grey indicate how many pixels show a particular $\Delta D$ for a given dark current. As in the graphs for the SBIG camera, Fig. 3.4 is divided in areas of 0.1 counts/s by 50 counts. 

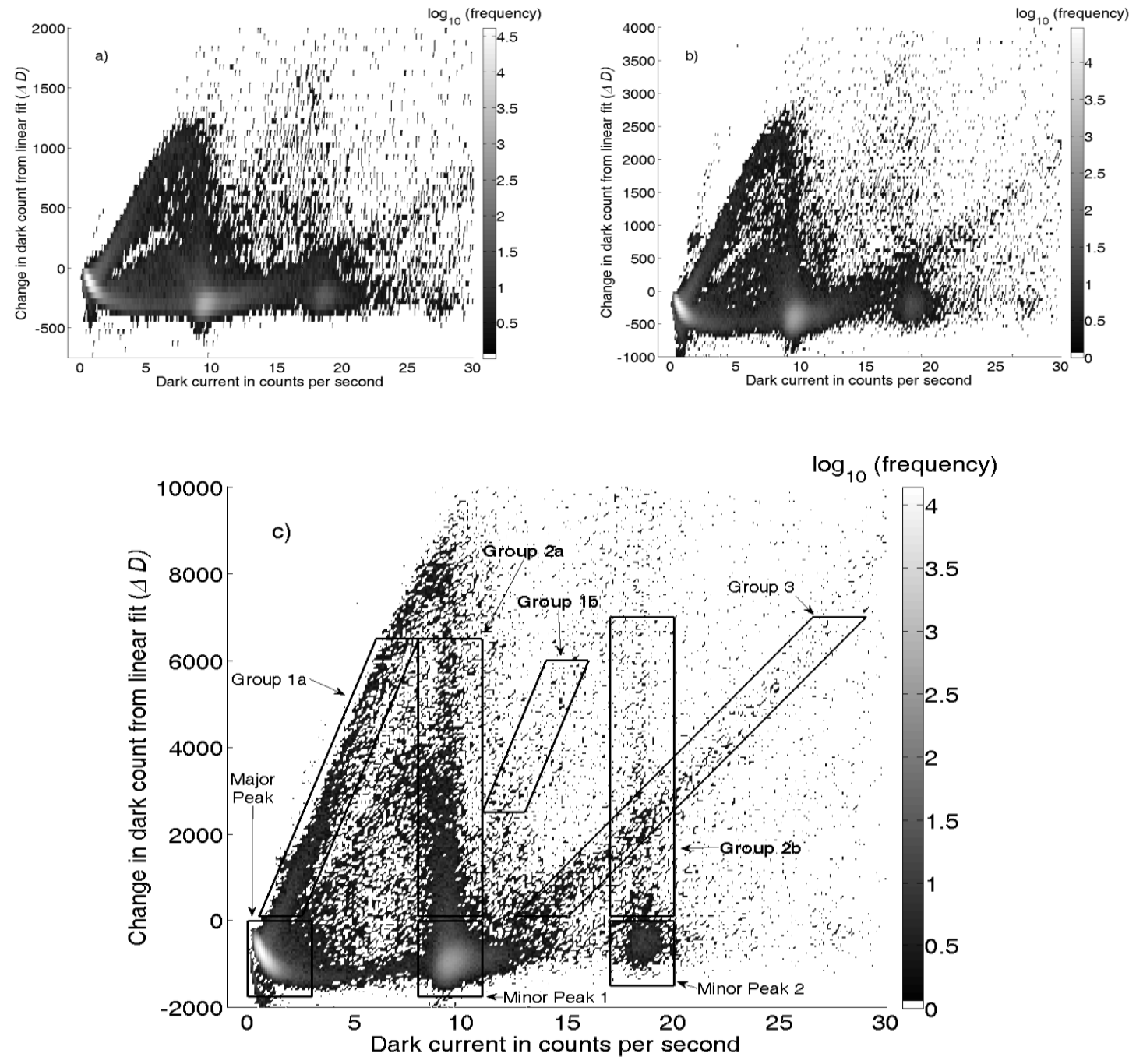

Fig. 3.4: Dark current from linear fit versus the change in dark count, $\Delta D$, for the KAF-0400 at $278 \mathrm{~K}$ evaluated at (a) $640 \mathrm{~s}$, (b) $900 \mathrm{~s}$, (c) $1800 \mathrm{~s}$.

The different panels in Fig. 3.4 clearly show that the KAF-0400 chip shows distinct groups as well. As one increases the exposure time at which $\Delta D$ is evaluated, groupings become more distinct. We therefore used the largest exposure time of $1800 \mathrm{~s}$ (Fig. 3.4c) to define the different groups. Analogous to the KAF-1602E sensor, the KAF-0400 sensor has groups similar to Group 1, Group 2, Major Peak, and Minor Peak as in Fig. 
3. The Major Peak for the KAF-0400 contains approximately 323,954 pixels, corresponding to about $82.4 \%$ of the chip. Pixels in the Major Peak have low dark current and, unlike for the KAF-1602E sensor, a negative $\Delta D$. Instead of a single Minor Peak, Fig. 3.4 shows two minor peaks that are also located at negative $\Delta D$ values. Minor Peak 1 (35,638 pixels) is located at about 9 counts/s and Minor Peak 2 (1,741 pixels) at about 18 counts/s. Group 1a (4,336 pixels) and Group 2a (6,203 pixels) mirror the characteristics of Group 1 and Group 2 in the KAF-1602E. Group 1b (373 pixels) and Group 2b (1,538 pixels) share the same shape as Group 1a and Group 2a, but are shifted to the right. Group $1 \mathrm{~b}$ emerges from the Minor Peak 1 and connects with Group 2b, pixels that have the same dark current as Minor Peak 2 pixels but with various changes in dark count $\Delta D$. Another set of pixels emerges from Minor Peak 1 with a smaller change in dark count than Group 2a pixels.

Fig. 3.5 illustrates the behavior of a typical pixel representing each group. Pixel 1a, 1b, $2 \mathrm{a}, 2 \mathrm{~b}$, and 3 all belong to their respective group name, and as one would expect, pixels from these groups show various degrees of nonlinearity in the dark current with long exposure times. Pixel 6, a hot pixel located in Minor Peak 1, is shown to have a large, mostly linear, dark current in Fig. 3.5a. Pixel 5 is located in Minor Peak 2 and has an even larger increase in dark count. Pixel 4 is a typical example of a regular pixel located in the Major Peak.

From Fig. 3.5a it appears that pixels have either a linear time dependence of the dark count (pixels in the three peaks) or a negative curvature (other pixels). However, a 
closer examination of the short exposure times, as shown in Fig. 3.5b, reveals that all pixels initially have a positive curvature. This positive curvature explains why fits done at short exposure times to obtain Fig. 4 result in a negative baseline for $\Delta D$ where the three peaks are located. The initial smaller slopes at low exposure times will cause the extrapolated fits to predict smaller dark count values for all pixels if it would not be for the later occurring negative curvatures for some pixels.
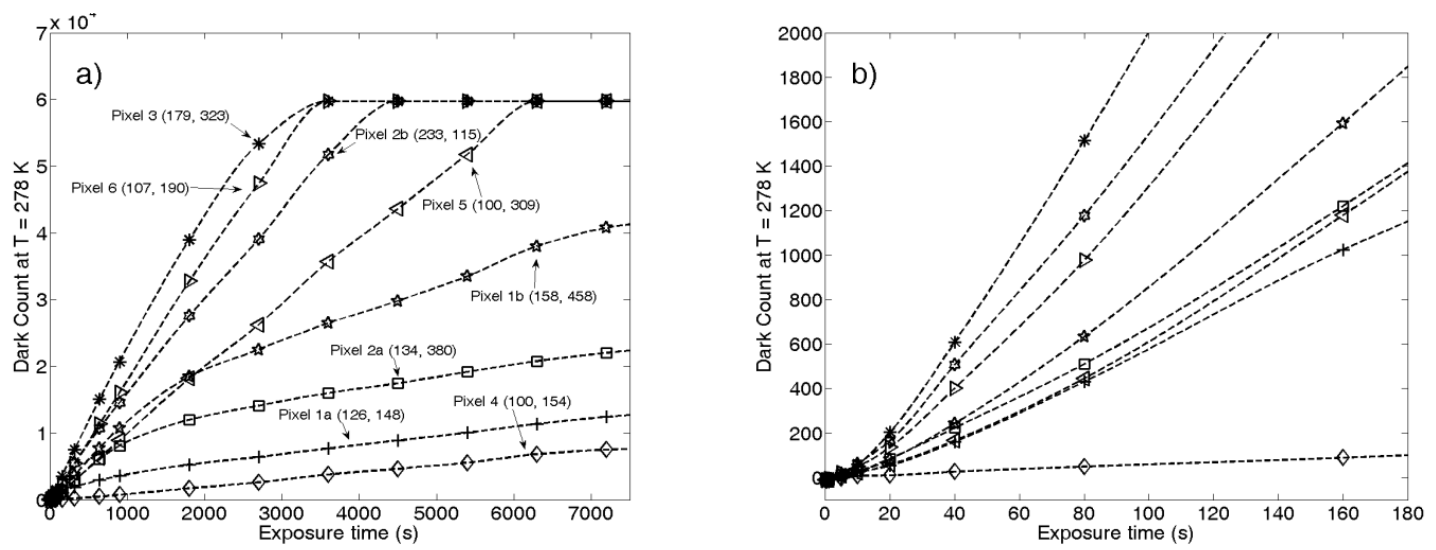

Fig. 3.5: Dark count at $278 \mathrm{~K}$ as a function of exposure time for eight pixels for the KAF-0400. (a) Exposure times up to 7200 s. (b) Enlargement with exposure times up to $180 \mathrm{~s}$.

\subsection{Discussion}

Dark current is the thermally assisted excitation of electrons from the valence into the conduction band. From the dark current distribution of an individual dark frame one can find two distinct peaks for the KAF-1602E and three peaks for the KAF-0400. The first peak contains the majority of pixels for each camera. These pixels are devoid of any midgap impurity that causes the high dark current of hot pixels. The KAF-1602E chip appears to have one type of impurity which causes a number of pixels (1.1\%) to become 
hot (Minor Peak). The KAF-0400 sensor has a larger fraction of hot pixels with $9.1 \%$ located in the Minor Peak 1. The larger number of impurities increases the likelihood of finding two impurities in the same pixel possibly giving rise to a smaller, but distinct, second peak (Minor Peak 2). One would expect that an impurity produces dark current at the same rate, filling a pixel's well and causing a linear pixel count until full well is reached. However, the data presented above clearly shows that this is not true for all pixels. With increasing exposure time, pixels in Group 2 stop being hot and start to behave more like pixels without midgap impurities. In a sense, the impurity that is present becomes inactive. To explain this phenomenon we need to look in more detail at the design of a CCD sensor.

Electrons excited into the conduction band either by photons or through thermal energy are actively collected only within the potential well. Front-illuminated imagers generally have a large field free region where electrons are not actively collected. Free electrons generated in the field-free part of the imager have to diffuse first toward the potential well before being collected and counted. Depending on the life time, some electrons will recombine with a hole before collection or diffuse into adjacent pixels. Hence, electrons generated at impurity sites outside the potential well will not contribute to the dark count in the same amount as electrons that are generated by an impurity in the well. The edge of the potential well is not static throughout the exposure. The depletion edge moves closer to the front surface with increasing number of electrons collected in the well. Impurities located close to the depletion edge can therefore move from being within the well at the beginning of the exposure to being in 
the field-free region at the end of the exposure. Since the collection efficiency of electrons generated outside the well is lower than the efficiency within the well, the rate at which these impurities generate dark current decreases with increasing exposure time. This phenomenon can explain the negative curvature of several pixels in Fig. 3.2 and Fig. 3.5. Figs. 3.3 and 3.4 could therefore be used to get information on an impurity's location. Major Peak pixels are devoid of any midgap impurity generating an intrinsic low dark count. Minor Peak pixels contain one impurity causing an increase in dark current. The impurity is located well within the depletion region and therefore generates dark current at a constant rate until the pixel reaches saturation. Hot pixels, including Minor Peak 1 pixels are randomly distributed across the chip. Assuming a random distribution of impurities that cause a pixel to become hot, it would be expected that some pixels have two impurities. Two hot impurities can give rise to the pixels located in Minor Peak 2 for the KAF-0400. Due to the smaller number of Minor Peak pixels in the KAF-1602E, having two impurities in one pixel is less likely and a second peak is barely, or arguably not, visible in Fig. 3.3. We hypothesize that Group 1 and Group 2 pixels have impurities close to the depletion edge. The lower the dark current ( $\mathrm{x}$-axis in Fig. 3.3 and 3.4) as obtained from the low exposure time fits, the faster the impurity moves outside the depletion region. Hence, Group 1 pixels, with an impurity very close to the initial depletion edge, have different dark current values even for low exposure times. The dark current for all Group 1 pixels decreases such that the longer exposure times (such as at $640 \mathrm{~s}, 900 \mathrm{~s}$ and $1800 \mathrm{~s}$ in Figs. 3.3 and 3.4) yield a smaller dark count than expected from the linear fit. 
The behavior of Group 2 pixels is similar. Again, for the long exposure times the dark current decreases because the impurity has moved out of the depletion region. At the short exposure times used for the fit, the impurity is still within the well and the dark current is therefore the same as for Minor Peak pixels. The same explanation can be applied to Group 1a and Group 2a pixels in the KAF-0400 sensor. Due to the larger number of impurities one also finds pixels with one impurity within the depleted well and one impurity at the initial depletion edge resulting in Group $1 \mathrm{~b}$ and Group $2 \mathrm{~b}$. The occurrence of Groups 3 in both chips is believed to be the result of further distinction within the peaks themselves but is not well understood at this point. The global positive curvature for the KAF-0400 sensor at short exposure times must be specific to the chip design which results in a pathway for thermally generated electrons to slowly activate with short exposure times. More work needs to be done to explain this phenomenon.
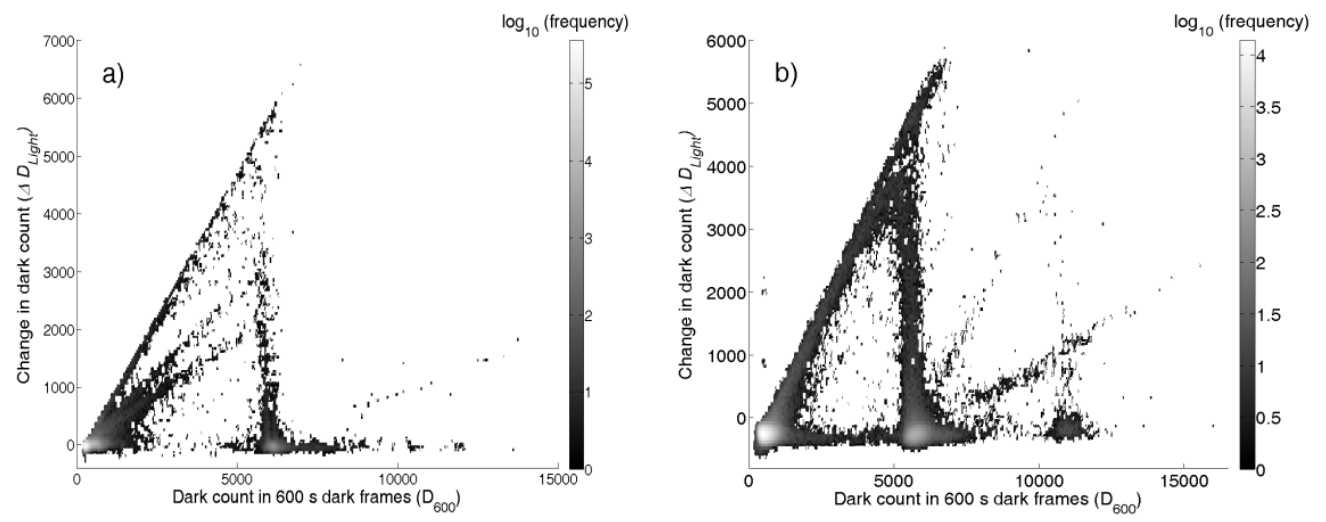

Fig. 3.6: Change of dark count after illumination versus dark count of a $600 \mathrm{~s}$ dark frame [12]. (a) KAF-1602E for an average initial count due to the light exposure of approximately 26,000 at $288 \mathrm{~K}$.(b) KAF-0400 for an average initial count due to the light exposure of approximately 42,100 at $278 \mathrm{~K}$. 
If filling the potential well with thermally excited electrons causes the depletion region to shrink and to move some impurities out of the well, the same should happen if one fills the well with photoelectrons. We found that this is indeed the case [27]. Fig. 3.6 shows the change in dark current after an imager was exposed to an initial brief flash of light. Fig. 3.6 was generated in a manner similar to Figs. 3.3 and 3.4. Here the x-axes show the dark count in a $600 \mathrm{~s}$ dark frame and the y-axes show the difference between the dark count of a regular dark frame and a frame that has been illuminated with light. For example, one would expect that a Group 1 pixel would produce very little dark current if the well was filled with photoelectrons causing the impurity to sit outside the depletion edge. This is found to be true; for example, Pixel 1 for the KAF-1602E has a dark count of 1,589 counts at the $600 \mathrm{~s}$ dark frame, but only a dark count of 239 counts if illuminated with light (approximately 26,000 counts as is shown in Fig. 3.6). The resulting change in dark count is therefore almost as large as the dark count resulting in a pixel with $D_{600}=1,589$ and $\Delta D_{\text {Light }}=1,349$. Hence, the initial flash of light suppresses the dark current almost entirely. Group 1 pixels are therefore located along a line where $\Delta D_{\text {Light }}$ is almost as large as $D_{600}$. More generally, it is apparent from Fig. 3.6 that the identified groups occur for both cameras for the light exposure analysis as well.

\subsection{Conclusion}

Previous work on dark current in digital imagers has focused on its temperature dependence. Using temperature data one can, for example, find the activation energy characteristic of a particular type of impurity. We found that the nonlinear time dependence of dark current can point to an impurity's location. An impurity located 
near the depletion edge will not produce a dark count at a steady rate. Scaling the dark count of pixels with such impurities is therefore problematic. Since the dark current depends on the number of already collected electrons, dark count correction becomes especially complicated if one considers that during an exposure, electrons are generated thermally as well as through photoexcitation. Our study shows that accurately predicting the dark count of certain pixels necessitates knowing how the depletion edge moved during a particular exposure. 


\section{CHAPTER 4 - CMOS DIGITAL IMAGER NONLINEARITIES}

\subsection{Introduction}

The pixel architecture of CCD and CMOS pixels are similar enough that there is no reason to expect the nonlinear behavior observed in the Kodak CCD imagers to be exclusive to CCD architecture. The model would predict that a CMOS pixel, sharing the basic architecture of a CCD pixel, complete with impurities, may obtain an impurity near the depletion edge. Thus, these pixels would also show a nonlinear response to exposure time as well as a reduction in dark current when exposed to illumination.

\subsection{Experimental setup}

Data was taken with a 2 megapixel CMOS imager, the CMV2000, [28] from CMOSIS Image Sensors corporation. The CMV2000 has 2048 x 1088 pixel resolution with $5.5 \times 5.5 \mu^{2}$ pixels. CMOSIS hopes to compete in low-cost, high frames-per-second applications and provided an imager to our lab for testing to characterize the dark current of the imager.

Single dark frames were taken using the CMOSIS imager and camera with exposure times from $0.0042 \mathrm{~s}$, the shortest exposure time allowed by the software, to $60 \mathrm{~s}$ at a temperature of $5{ }^{\circ} \mathrm{C}$. Longer exposure times would have been preferable to observe non-linear behavior across a larger set of pixels, however pixels that approached and reached saturation were detected within this regime. It is conjectured that due to the presence of these large dark current pixels, the test software was imposed with a 
limitation of a maximum exposure time of $62 \mathrm{~s}$. The camera and imager were placed in a temperature controlled box with a fan to circulate air in the enclosure. The temperature was controlled using a piezoelectric cooler, and allowed to stabilize prior to taking each frame.

\subsection{Results}

The CMV2000 CMOS imager does have pixels that behave nonlinear with respect to exposure time well below saturation. Figure 4.1 is indicative of this behavior, featuring a plot of the change in dark current $(\Delta D)$ with respect to the slope $(m)$ of the pixels at low exposure times calculated from the frames taken from $1 \mathrm{~s}$ to $5 \mathrm{~s} . \Delta D$ was calculated by extrapolating the dark current production at the low exposure times out to $50 \mathrm{~s}$ and then subtracting off an actual frame taken at $50 \mathrm{~s}$. Pixels in the CMOSIS imager exhibiting nonlinear behavior were observed and are similar to the Group 1 pixels observed in the Kodak imagers. The region loosely corresponding to these type of pixels, are labeled as "Group 1 Type" in Fig. 4.1 along with "Hot Linear" pixels, those that have large dark current signal but do not exhibit non-linear exposure time behavior. 


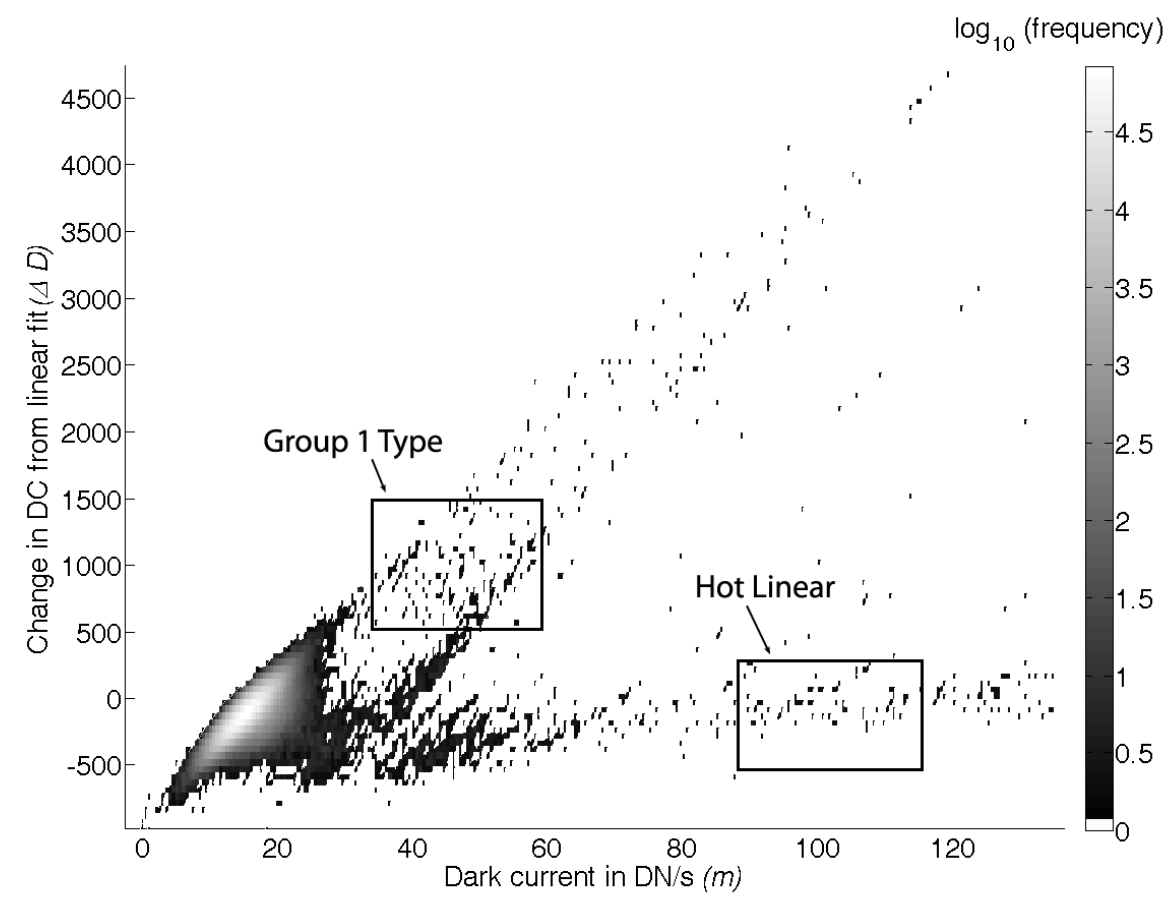

Fig. 4.1- Change in dark current $(\triangle D)$ between slopes $(m)$ after $5 \mathrm{~s}$ extrapolated out to 50 s (or to a fixed maximum count of the nearest frame to 1800 counts) at $278 \mathrm{~K}$.

Figure 4.2 details dark frame counts versus exposure time for six of the nonlinear pixels picked from the region labeled "Group 1 Type" in Fig. 4.1. These pixels exhibit significant dark current at low exposure times but dark current is diminished at later exposure times. Additionally, for reference, Fig. 4.2 features a Main peak type pixel with a $\Delta D$ value of about $0 \mathrm{DN}$ and low dark current. This pixel is represented by a diamond in the figure. 


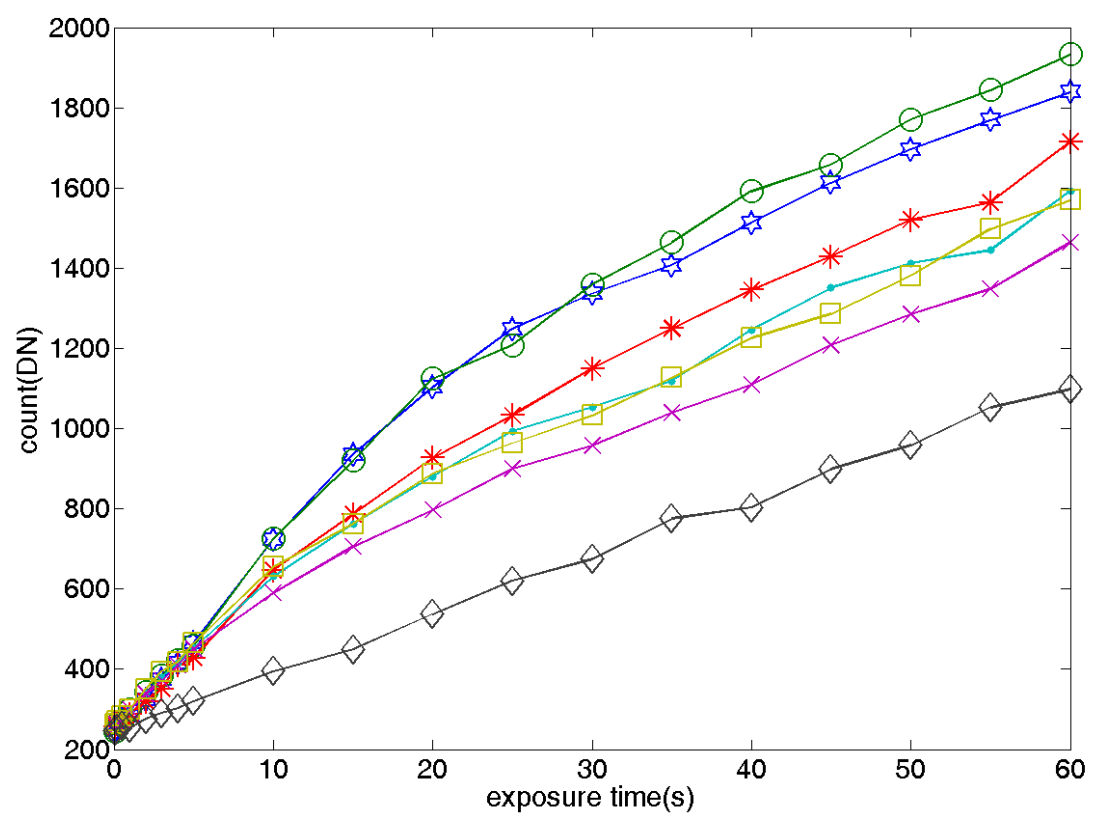

Fig. 4.2- Dark count at $278 \mathrm{~K}$ as a function of exposure time for seven pixels for the CMOSIS. Six Group 1 type pixels with an $m$ value between 35 and $55 \mathrm{DN} / \mathrm{s}$, and a $\Delta D$ value between 50 to $1500 \mathrm{DN}$ as seen in Fig. 4.1, as well as a single Main peak pixel (diamond marker) with a $\Delta D$ of about 0 DN.

A peculiarity observed in the dark frames for the hot pixels that do reach saturation is an inconsistent saturation level. Figure 4.3 displays five pixels from the group labeled "Hot Linear" in Fig. 4.1. While all of these pixels show linearity up until saturation, the dark current saturation levels are below the saturation levels found when taking light exposures where the pixels uniformly saturated 4095, counts. Indeed, the dark current only saturation levels for these pixels are all around 2500 counts, but have a unique saturation level for each pixel. One of the advantages of CMOS imagers is the ability to build in more complex signal processing on a pixel-by-pixel level. We can speculate to the cause of the non-uniform saturation levels as being due to a region of 
each pixel devoted to sampling against to subtract off dark current caused by such things as non-uniform temperature throughout the imager. The possibilities for how an impurity in the various regions of the pixel are intriguing, however, we are not currently able to obtain more information from the manufacture of the imager and a more detailed analysis is beyond the scope of this investigation.

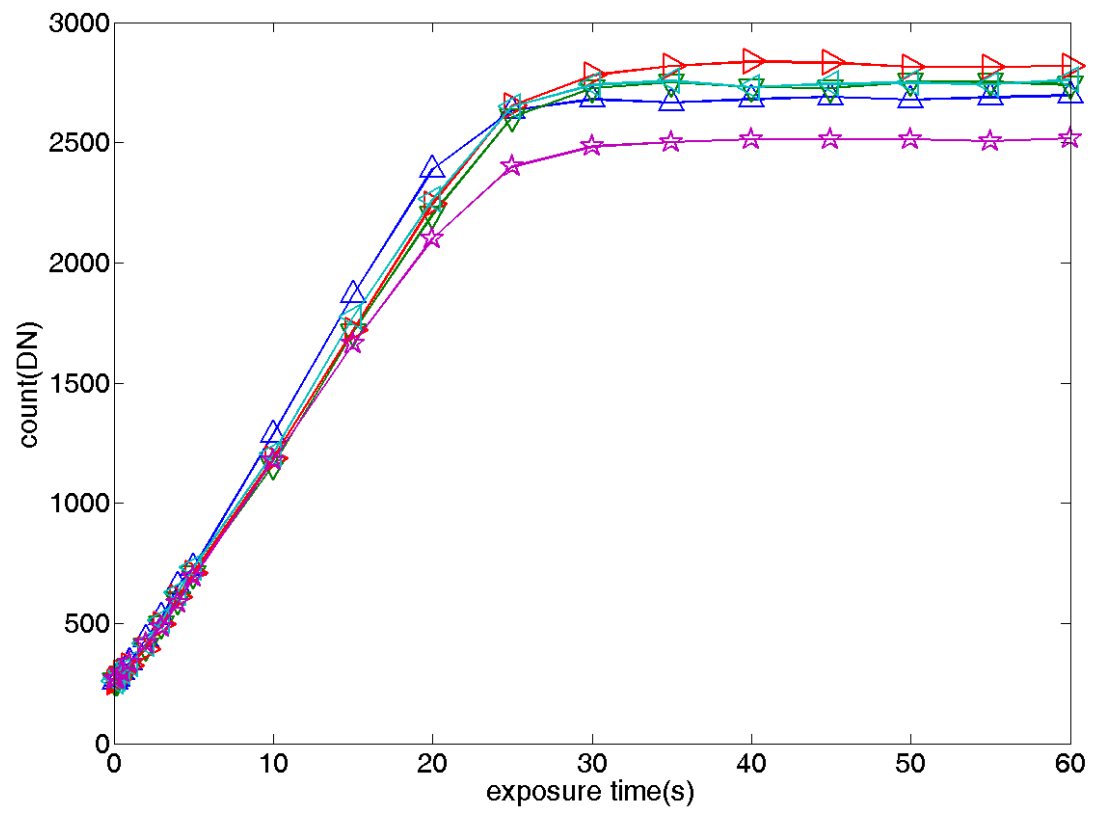

Fig. 4.3- Dark count at 278K as a function of exposure time for five hot pixels from the CMOSIS imager with variation in saturation level.

\subsection{Summary}

While the majority of the pixels in the CMOSIS CMOS imager exhibit linear behavior, there are still an abundance of large dark current, often called "hot", pixels and further, there are pixels that exhibit nonlinear dark signal with respect to exposure time. These pixels confirm that the nonlinear type pixels are not unique to CCD chip architecture and provide evidence that the model of an impurity near a moving 
depletion region can explain the presence of such pixels as these are features that would be expected to be present for both CCD and CMOS imagers. 


\section{CHAPTER 5 - MODELING OF DARK CURRENT IMPURITIES AND NONLINEAR GENERATION RATES}

\section{$5.1 \quad$ Introduction}

A model explaining the observed nonlinear time dependence of pixels in CCD pixels is presented in this section. The model describes the movement of a pixel's depletion edge as a function of varying quantities of signal charge, the charge in the potential well. Dynamic depletion edges will affect dark current collected by pixels with impurities located in the region of movement of the edge. The model attempts to address nonlinear behavior of dark current with respect to exposure time well below the saturation level seen in CCD imagers as discussed in Chapter 3 as well as in CMOS imagers as discussed in Chapter 4. Modeling an imager by giving pixels varying number of impurities and depths of those impurities, assuming a uniform distribution, leads to characteristic behavior observed in the imagers. A major portion of the research presented in this section has been published in [29].

CCD pixels are generally assumed to generate a linear count both in their response to light and in their dark current generation. Dark frames with a longer exposure time provide for a frame with higher signal-to-noise than a frame at a shorter exposure time and therefore it is common practice to use dark frames that have been scaled down to the required integration time for dark frame subtraction. This method of dark frame subtraction is reliant upon the linearity of the dark count generation. The presence of a minority of pixels showing a nonlinear response to exposure time is a complication for this method of correction. Prior studies have shown the presence of this type of pixel [26]. Shown in Fig. 5.1 is the dark count as a function of exposure time of a selection 
of five pixels on a KAF-1602E CCD sensor in a SBIG ST-8XE camera. Included in this selection are pixels showing a nonlinear response to exposure time such as the pixel labeled Pixel 1. Displayed in Fig. 1b is the difference, $\Delta D$, between a linear fit of dark count values of exposure times between 5 to $60 \mathrm{~s}$ evaluated at $640 \mathrm{~s}$ minus the actual dark count for an exposure of $640 \mathrm{~s}$.
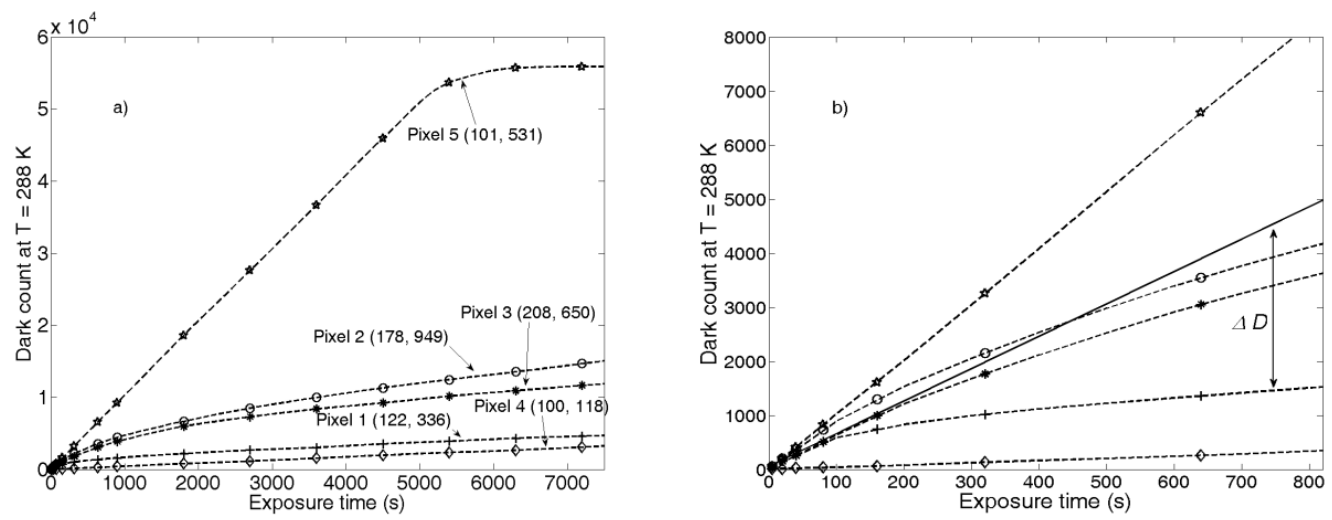

Fig. 5.1: Dark count at $288 \mathrm{~K}$ as a function of exposure time for 5 pixels for the KAF-1602E sensor. (a) Exposure times up to $7200 \mathrm{~s}$. (b) Enlargement with exposure times up to $800 \mathrm{~s}$ and linear fit to Pixel 1 (same pixels shown as in (a)).[26]

In this section, we introduce a model that assumes a uniform distribution of impurity defects in the imager. The impurities are distributed with varying frequency and depths within the pixels and the model includes a dynamic depletion edge as a result of signal charge collection. These attributes result in modeled pixels that have a nonlinear generation of depletion dark current with respect to exposure time. The model indicates another important finding, that impurity location will be crucial to the quantity of dark current produced. Dark current generation in the presence of a dynamic depletion edge will be dependent upon the collected signal charge. The moving depletion edge will have the effect of "turning off" impurities in the sense that 
the pixel will no longer collect most of the dark current electrons generated by an impurity that is no longer within the depletion region. Thus, the location of dark current sources will determine how the pixel's collected dark current will respond to increasing signal charge.

Using impurity concentrations and fundamental electrostatics, depletion depth calculations are well explored $[2,6]$. We assume a Gaussian distribution of n-type donor atoms and follow the work of Taylor, et al. [30] in calculating the depletion depth for a CCD pixel.

Figure 5.2 displays a schematic diagram of the moving depletion edge, located at a distance $\omega$ from the oxide, in a CCD pixel. An impurity, marked with an $\mathrm{X}$ in Fig. 5.2, will be located within the depletion region when the collection region contains no signal charge; however, as charge is collected the depletion edge moves and effectively shifts the impurity out of the collection region. The region of collection is taken to include the entire well from the surface to the depletion edge plus a small distance, $\delta$, where it is likely that a fraction of the carriers generated by this impurity are also collected in the pixel. Thermally generated electrons are still produced by impurities outside of the collection region. However, in this region the generation rate is much less than the depletion generation rate at assumed operating temperatures. In addition, it is conjectured that there will be a region where some of the generated dark current is lost when an impurity is located precipitously close to the depletion edge. For simplicity, this distance is assumed to be the same $\delta$. 


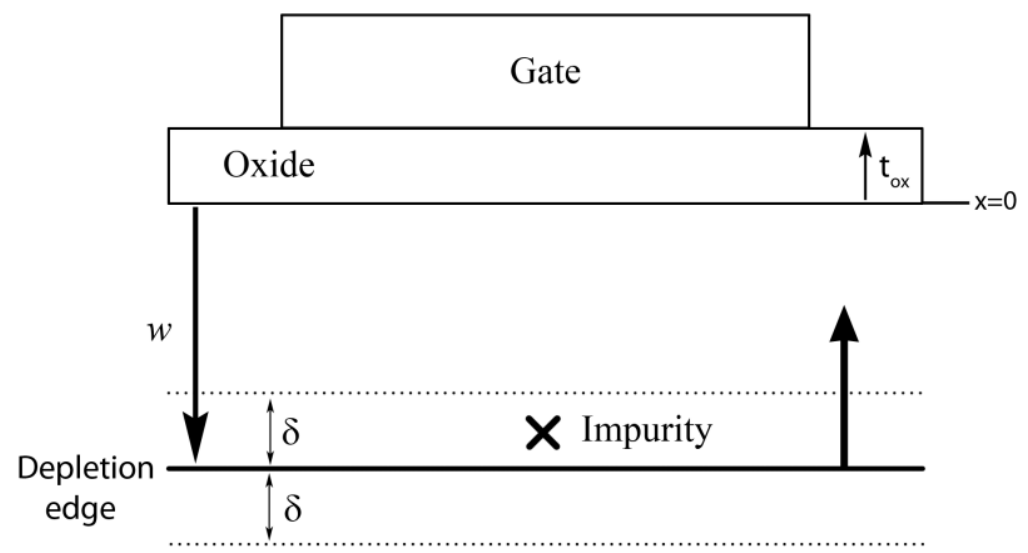

Fig. 5.2- Moving depletion region in a CCD pixel

Finally, we use the model to generate data to show an overview of the modeled pixels' nonlinear dark current production with respect to exposure time across the whole modeled imager. Data generated using the model and experimental data from imagers displayed in Chapter 3 are compared.

This model, along with accurate data from the manufacturing process and experimental data from an imager, would be capable of determining the depths of impurities within the pixels of that imager. This knowledge would provide for further clarification of the manufacturing process and could highlight the source of defects caused by different steps in the process.

\subsection{Model Description}

\subsubsection{Dynamic depletion edge}

To estimate the depletion edge as a function of the signal charge in the CCD well, we assume a Gaussian profile for the n-type buried channel implant into p-type silicon so that: 


$$
N_{d}(x)=K \exp \left(-\frac{(x-r)^{2}}{2 \sigma^{2}}\right)
$$

where $N_{d}$ is the donor density and $K$ is the peak concentration which can be calculated using the total implant dose, $Q_{c d}$, as discussed below. In addition, $r$ and $\sigma$ are the range and straggle respectively, which characterize the implant profile, and $x$ is the distance into the silicon. We require the solution to Poisson's equation for the oxide and for three different regions in the semiconductor. The three regions in the semiconductor are 1) the surface region $\left.\left(0<x<x_{1}\right), 2\right)$ the channel region $\left(x_{1}<x<x_{2}\right)$, and 3$)$ the back region $\left(x_{2}<x<\omega\right)$. Figure 5.3 shows the absolute value of the net impurity concentration as a function of depth in the modeled pixel using the parameters in Table 5.1 and containing a signal charge of $5.7 \times 10^{11} \mathrm{e}-/ \mathrm{cm}^{2}$. The corresponding locations of $x_{1}$ and $x_{2}$ are also labeled on the figure. The metallurgical junction, where the material transitions from n-type to p-type, is at about $1 \times 10^{-4} \mathrm{~cm}$.

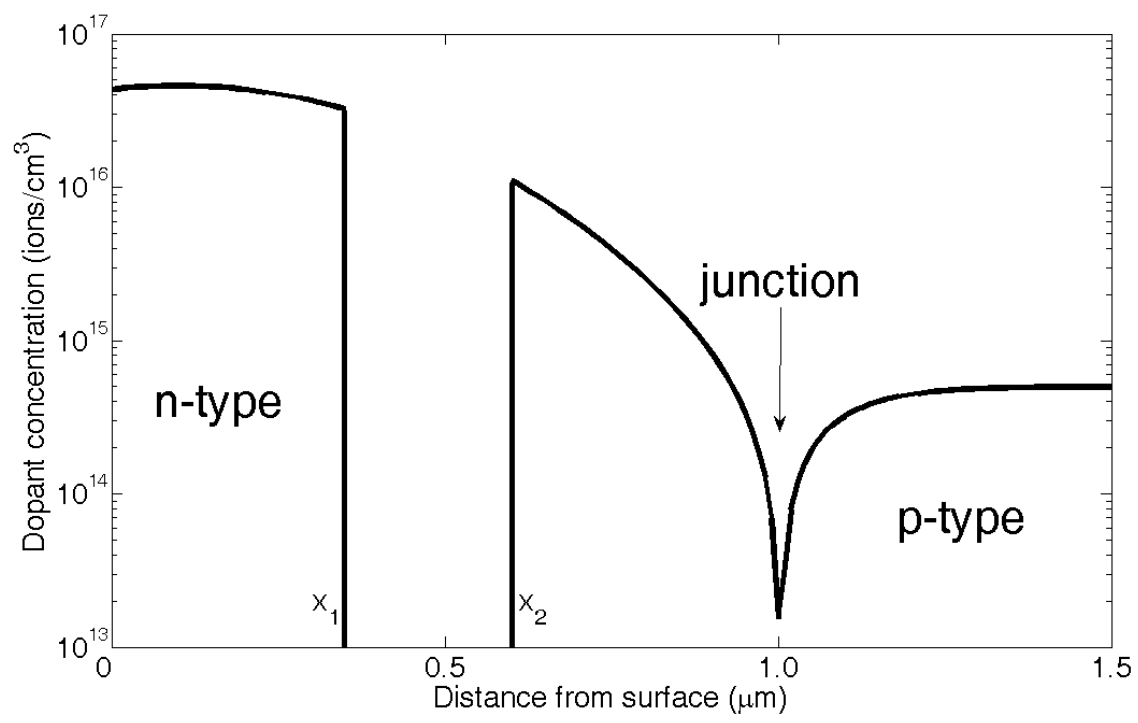

Fig. 5.3- Dopant concentration as a function of depth from the surface of the modeled pixel, shown with a partially filled well containing a signal charge of $5.7 \times 10^{11} \mathrm{e}-/ \mathrm{cm}^{2}$ 
In the oxide, Poisson's equation gives:

$$
\nabla^{2} \psi_{o x}=0
$$

In the semiconductor we have the charge density:

$$
\rho(x)=q\left(N_{d}(x)-N_{a}\right)
$$

where $N_{a}$ is the uniform p-type doping concentration of the substrate, $N_{d}$ is the donor concentration as given in Eq. 5.1, and $q$ is the elementary charge. Poisson's equation in this region is then:

$$
\nabla^{2} \psi_{s}(x)=-\frac{\rho(x)}{\varepsilon_{S i}}=\frac{q}{\varepsilon_{S i}}\left(N_{a}-N_{d}(x)\right)
$$

where $\varepsilon_{s i}$ is the permittivity constant in silicon. Following the methods of Taylor et al. [30], solving for the potential in the oxide gives:

$$
\Psi_{o x}(x)=\alpha+\beta x
$$

where $\alpha$ and $\beta$ are constants. Poisson's equation in the silicon is:

$$
\nabla^{2} \psi_{s}(x)=\frac{q}{\varepsilon_{S i}}\left(N_{a}-N_{d}(x)\right)
$$

Integrating once with respect to $x$ yields:

$$
\nabla \psi_{s}(x)=\frac{q}{\varepsilon_{S i}}\left(N_{a} x+C-\int^{x} d \xi N_{d}(\xi)\right)
$$

where $C$ is a constant of integration, and the integral of the donor density is: 


$$
\int^{x} d \xi N_{d}(\xi)=K \sqrt{\frac{\pi}{2}} \sigma \operatorname{Erf}\left(\frac{x-r}{\sqrt{2} \sigma}\right),
$$

It can be observed here that setting equation Eq. 5.8 equal to $Q_{c d}$, the buried channel dose, will allow for the solving of $K$ when the limits of integration are taken to be from $\mathrm{x}=0$ to $\mathrm{x}=$ infinity.

Integrating once more with respect to $x$ gives:

$$
\psi_{s}(x)=\frac{q}{\varepsilon_{s i}}\left(\frac{N_{a}}{2} x^{2}+C x+D-K \sqrt{\frac{\pi}{2}} \sigma \int^{x} d \xi \operatorname{Erf}\left(\frac{\xi}{\sqrt{2} \sigma}\right)\right)
$$

where $D$ is the constant of integration and the integral in the last term can be solved to yield:

$$
\begin{gathered}
\int d \xi \operatorname{Erf}\left(\frac{\xi}{\sqrt{2 \sigma}}\right)=\sqrt{2} \sigma \int d \xi \operatorname{Erf}(\xi)=\sqrt{2} \sigma[\xi \operatorname{Erf}(\xi)+ \\
\left.\frac{1}{\sqrt{\pi}} e^{-\xi^{2}}+\Gamma\right]
\end{gathered}
$$

where $\Gamma$ is a constant of integration. We define:

$$
\gamma(z) \equiv z \operatorname{Erf}(z)+\frac{1}{\sqrt{\pi}} e^{-z^{2}} .
$$

The equation for the potential in the semiconductor for $\left(0<\mathrm{x}<\mathrm{x}_{1}\right)$ is therefore:

$$
\psi_{s}(x)=\frac{q}{\varepsilon_{S i}}\left(\frac{N_{a}}{2} x^{2}+C x+D-K \sqrt{\pi} \sigma^{2} \gamma\left(\frac{x-r}{\sqrt{2} \sigma}\right)\right),
$$

where $\Gamma$ has been combined with $D$, and: 


$$
\nabla \psi_{s}=\frac{q}{\varepsilon_{S i}}\left(N_{a} x+C-K \sqrt{\frac{\pi}{2}} \sigma E r f\left(\frac{x-r}{\sqrt{2} \sigma}\right)\right) .
$$

The boundary conditions are as follows:

$$
\begin{gathered}
\psi_{o x}(-t)=V_{g}=\alpha-\beta t_{o x} \\
\psi_{o x}\left(0^{-}\right)=\psi_{s}\left(0^{+}\right) \\
\varepsilon_{o x} \psi_{o x}^{\prime}\left(0^{-}\right)=\varepsilon_{s i} \psi_{s}^{\prime}\left(0^{+}\right), \\
\psi_{s}\left(x_{1}\right)=V_{c h} \\
\psi_{s}^{\prime}\left(x_{1}\right)=0
\end{gathered}
$$

where $\varepsilon_{o x}$ is the oxide permittivity and $t_{o x}$ is the thickness of the oxide. The first boundary condition merely states the voltage at $x=-t_{o x}$ is equal to the voltage applied to the gate. The second and third conditions require that the potential and the electric displacement be continuous across the interface between the oxide and the silicon. The fourth and fifth conditions require that at the edge of the channel region the potential will be a fixed value $\left(V_{c h}\right)$ and the field will be zero.

Our unknowns are: $\alpha$ and $\beta$ for the oxide potential, $C$ and $D$ for the semiconductor potential and $x_{1}$, the position of the edge of the channel closest to the surface. Using the boundary conditions we arrive at: 


$$
\begin{aligned}
\frac{N_{a}}{2} x_{1}{ }^{2}+\frac{t_{o x} \varepsilon_{S i}}{\varepsilon_{o x}} & N_{a} x_{1}+K \sigma^{2}\left(e^{-\left(x_{1}-r\right)^{2} / 2 \sigma^{2}}-e^{-r^{2} / 2 \sigma^{2}}\right) \\
- & K \sqrt{\frac{\pi}{2}} \sigma\left(\frac{t_{o x} \varepsilon_{S i}}{\varepsilon_{o x}}+r\right)\left(\operatorname{Erf}\left(\frac{x_{1}-r}{\sqrt{2} \sigma}\right)+\operatorname{Erf}\left(\frac{r}{\sqrt{2} \sigma}\right)\right) \\
+ & \frac{\left(V_{c h}-V_{g}\right) \varepsilon_{S i}}{q}=0 .
\end{aligned}
$$

This equation cannot be explicitly solved for $x_{1}$ and therefore it is necessary to find the value of $x_{1}$ which satisfies the equation by numerical methods. Once $x_{1}$ has been determined, then $\alpha, \beta, C$, and $D$ may be computed. Therefore, the solution for the potential on the surface side of the device (i.e. $0<x<x_{1}$ ) has been found.

In the channel region of the device $\left(x_{1}<x<x_{2}\right)$, the signal charge just compensates for the ionized donor impurity distribution. Therefore, the field is zero and the potential is constant at $V_{c h}$ in this region.

Finally, for the back side of the channel, $x_{2}<x<\omega$, we again have to solve Poisson's equation:

$$
\nabla^{2} \psi_{s}(x)=\frac{q}{\varepsilon_{S i}}\left(N_{a}-N_{d}(x)\right)
$$

The solution is as before, except we require two new constants of integration $A$ and $B$. The first and second integral with respect to $x$ yield respectively:

$$
\begin{gathered}
\nabla \psi_{s}(x)=\frac{q}{\varepsilon_{S i}}\left(N_{a} x+A-K \sqrt{\frac{\pi}{2}} \sigma \operatorname{Erf}\left(\frac{x-r}{\sqrt{2} \sigma}\right)\right), \\
\psi_{s}(x)=\frac{q}{\varepsilon_{S i}}\left(\frac{N_{a}}{2} x^{2}+A x+B-K \sqrt{\pi} \sigma^{2} \gamma\left(\frac{x-r}{\sqrt{2} \sigma}\right)\right) .
\end{gathered}
$$


With the boundary conditions now:

$$
\begin{gathered}
\psi_{s}\left(x_{2}\right)=V_{c h}, \\
\nabla \psi_{s}\left(x_{2}\right)=0, \\
\psi_{s}(\omega)=0, \\
\nabla \psi_{s}(\omega)=0 .
\end{gathered}
$$

Using the second and fourth boundary conditions and combining to solve for $\omega$ yields:

$$
\begin{gathered}
\omega=x_{2}+\left(\frac{K}{N_{a}} \sqrt{\frac{\pi}{2}} \sigma \operatorname{Erf}\left(\frac{\omega-r}{\sqrt{2} \sigma}\right)-\frac{K}{N_{a}} \sqrt{\frac{\pi}{2}} \sigma \operatorname{Erf}\left(\frac{x_{2}-r}{\sqrt{2} \sigma}\right)\right) \\
\cong x_{2}+\frac{K}{N_{a}} \sqrt{\frac{\pi}{2}} \sigma\left(1-\operatorname{Erf}\left(\frac{x_{2}-r}{\sqrt{2} \sigma}\right)\right) .
\end{gathered}
$$

To yield the final value we utilize the knowledge that $\omega$ is generally much larger than $r, \sigma$, and $x_{2}$, and therefore we can approximate $\operatorname{Erf}\left(\frac{\omega-r}{\sqrt{2} \sigma}\right) \cong 1$.

Employing the first and third boundary conditions and again combining the two we get

$$
\omega=\operatorname{sqrt}\left\{\begin{array}{c}
\frac{2 \epsilon_{S i} V_{c h}}{N_{a} q}+x_{2}^{2}+\frac{2 K}{N_{a}} \sqrt{\frac{\pi}{2}} \sigma r\left(\operatorname{Erf}\left(\frac{\omega-r}{\sqrt{2} \sigma}\right)-\operatorname{Erf}\left(\frac{x_{2}-r}{\sqrt{2} \sigma}\right)\right)+ \\
\frac{2 K \sigma^{2}}{N_{a}}\left(\exp \left(-\frac{\left(x_{2}-r\right)^{2}}{2 \sigma^{2}}\right)-\exp \left(-\frac{(\omega-r)^{2}}{2 \sigma^{2}}\right)\right)
\end{array}\right\}
$$


We can again approximate $\operatorname{Erf}\left(\frac{\omega-r}{\sqrt{2} \sigma}\right) \cong 1$ and for the same reasons can approximate $\exp \left(-\frac{(\omega-r)^{2}}{2 \sigma^{2}}\right) \cong 0$. Our expression for $\omega$ in the equation above then simplifies to:

$$
\sqrt{\frac{2 \epsilon_{S i} V_{c h}}{N_{a} q}+x_{2}^{2}+\frac{2 K}{N_{a}} \sqrt{\frac{\pi}{2}} \sigma r\left(1-\operatorname{Erf}\left(\frac{x_{2}-r}{\sqrt{2} \sigma}\right)\right)+\frac{2 K \sigma^{2}}{N_{a}}\left(\exp \left(-\frac{\left(x_{2}-r\right)^{2}}{2 \sigma^{2}}\right)\right)} .
$$

We have two equations for $\omega$, equations 5.20 and 5.22. $x_{2}$ is still unknown, and solving for $x_{2}$ and $\omega$ individually from these two equations is not possible. To solve for $\omega$ for a given channel potential, we choose a value for $x_{2}$ that minimizes the differences between the two equations such that the difference in values for $\omega$ are less than $10^{-11} \mathrm{~cm}$.

The channel charge, $q_{s}$, is given by the integral of the dopant distribution between $x_{1}$ and $x_{2}$ so that:

$$
q_{s}=\int_{x_{1}}^{x_{2}} d \xi K \exp \left(-\frac{(\xi-r)^{2}}{2 \sigma^{2}}\right)=K \sqrt{\frac{\pi}{2}} \sigma\left(\operatorname{Erf}\left(\frac{x_{2}-r}{\sqrt{2} \sigma}\right)-\operatorname{Erf}\left(\frac{x_{1}-r}{\sqrt{2} \sigma}\right)\right)
$$

Thus for a given channel potential we can calculate both the collected signal charge in the channel and the corresponding depletion depth, the values needed to ultimately determine the relative location of the donor impurity sources to the depletion depth as charge is collected. 


\subsubsection{Modeling dark current production with a dynamic depletion edge}

A statistical argument is used in assigning impurities to pixels similar to the methods of McGrath et al.[6] If impurities are randomly distributed across the imager, the fraction of pixels, $P_{n}$, with a given number of impurities, $n$, can be calculated using a Poisson distribution such that:

$$
P_{n}=\left(\frac{N_{i m p}}{N_{p i x}}\right)^{n}\left[\frac{1}{n !} \exp \left(-\frac{N_{i m p}}{N_{p i x}}\right)\right],
$$

where $N_{i m p}$ is the total number of impurities present in the imager and $N_{p i x}$ is the total number of pixels on the imager. The number of pixels with $n$ impurities, $N_{n}$, is therefore $P_{n}$ multiplied by the total number of pixels, or:

$$
N_{n}=P_{n} N_{\text {pix }} .
$$

Our model assumes a uniform distribution of impurities as a function of depth within the device and assigns a randomized depth to each impurity. The algorithm therefore creates $N_{n}$ pixels with $n$ impurities and assigns depths for each of the impurities within each pixel.

To calculate the dark current generated by a pixel with a given integration time, our program first evaluates if all the impurities within that pixel will be located within the depletion area for the entire integration time. If so, the accumulated charge is simply calculated by multiplying the rate of dark current generation for the impurity, $R$, by the number of impurities and the integration time. This pixel will have a linear time dependence for the dark count. However, if this isn't the case, the program iteratively calculates the quantity of charge accumulated per step size of the integration time, $\Delta t$, 
by calculating the depth of the depletion edge with the previously accumulated signal charge. Then, for every impurity within the pixel, a check is done to see if the impurity remains within the region of collection. The quantity of new charge collected due to the impurity, $q_{n}$, is modified by where the impurity is within the region of collection. Once outside the depletion region, there is still a statistical probability that these electrons will be collected by the pixel and this is the motivation for defining the region, $\delta$, where a portion of the carriers created by an impurity just outside the depletion region will be collected by the pixel. There is also a statistical probability that some carriers generated by an impurity will be lost if the impurity is inside the depletion region but close to the depletion edge. For simplicity we combine these two effects. Thus, the quantity of new charge within a distance plus or minus $\delta$, is approximated by calculating the collected charge in these regions using:

$$
\Delta q_{n}=R \Delta t \frac{\left(w-x_{i m p}\right)+\delta}{2 \delta}
$$

for each time step up until the end of the integration time. If the impurity is within the depletion region and further than a distance $\delta$ from the depletion edge, the new charge is calculated by multiplying the rate by $\Delta t$. If the impurity is not located within the well, there is no charge collected for that impurity during that time. The effects of varying $\delta$ are shown later.

To convert between channel charge $q_{s}$ and digital counts, $D_{s}$, we use the relation:

$$
q_{s}=\frac{D_{s} g}{A}
$$


where $g$ is the gain in $\mathrm{e}^{-} / \mathrm{DN}$ and $A$ is the collection area of the pixel.

To summarize, the model first calculates a table of values relating depletion depth and signal charge based upon varying channel potentials. The model assigns depths and number of impurities within pixels and, using the aforementioned table, calculates the resulting dark current generated by those pixels for a given exposure time and initial signal count. The constants the model utilizes are typical of common imagers and are listed in Table 5.1. The parameters that can be thought of as dependent upon the camera system (and were therefore largely fixed) are the gate voltage, $V_{g}$, impact dose, $Q_{c d}$, oxide thickness, $t_{o x}$, number of acceptors per volume, $N_{a}$, relative oxide permittivity, $\varepsilon_{o x} / \varepsilon_{0}$, relative semiconductor permittivity, $\varepsilon_{s i} / \varepsilon_{0}$, range, $r$, straggle, $\sigma$, electron charge, camera gain, $g$, rate of dark current without impurity, $R_{0}$, rate of additional dark current due to a single impurity, $R_{l}$, pixel collection area, $A$, total pixels, $N_{p i x}$, full well count for a pixel, number of impurities, $N_{i m p}$, and $\delta$. Integration time is a variable that can be chosen by the user. 


\begin{tabular}{|c|c|}
\hline Variable & Value Chosen \\
\hline$V_{g}$ & $-5 \mathrm{~V}$ \\
\hline$Q_{c d}$ & $2.5 \times 10^{12} \mathrm{~cm}^{-2}$ \\
\hline$t_{o x}$ & $7.2 \times 10^{-6} \mathrm{~cm}^{-2}$ \\
\hline$N_{a}$ & $5.0 \times 10^{14} \mathrm{~cm}^{-2}$ \\
\hline$\varepsilon_{o x} / \varepsilon_{0}$ & 3.9 \\
\hline$\varepsilon_{s i} / \varepsilon_{0}$ & 11.7 \\
\hline$r$ & $1.0 \times 10^{-5} \mathrm{~cm}$ \\
\hline$\sigma$ & $3.0 \times 10^{-5} \mathrm{~cm}$ \\
\hline$g$ & $3.0 \mathrm{e}-/ \mathrm{DN}$ \\
\hline$R_{0}$ & $1.0 \mathrm{DN} / \mathrm{s}$ \\
\hline$R_{l}$ & $10.0 \mathrm{DN} / \mathrm{s}$ \\
\hline$A$ & $2.1 \times 10^{-7} \mathrm{~cm}$ \\
\hline$N_{p i x}$ & $1 \times 10^{6}$ \\
\hline Full well count & $65,536 \mathrm{DN}(16 \mathrm{bit})$ \\
\hline$N_{i m p}$ & $50,000 \mathrm{impurities}$ \\
\hline$\delta$ & $5.0 \times 10^{-6} \mathrm{~cm}$ \\
\hline $\begin{array}{c}\text { Integration } \\
\text { time }\end{array}$ & $500 \mathrm{~s}$ \\
\hline
\end{tabular}

Table 5.1- Typical parameter values used by the model

\subsubsection{Modeled nonlinear behavior}

To get a global overview of the nonlinearity of all of the modeled pixels, and to allow for comparison with experimental data, a linear fit of the type:

$$
D_{f i t}=m t,
$$

was applied to calculated dark count values for pixels using integration times of $5 \mathrm{~s}$ to $50 \mathrm{~s}$. Hence, the parameter $m$ represents the average generation rate in $\mathrm{DN}$ per second over this time interval. If it is assumed that the dark current is linear then the dark signal can be calculated at any exposure time, $t_{0}$, as $D_{f i t}\left(t_{0}\right)=m t_{0}$. This will be an accurate assumption for a pixel with an impurity fully within the depletion region, and therefore one that does not lose any signal due to the moving depletion edge 
However, for pixels with an impurity in a region that is affected by the moving depletion boundary, the effective dark current collected will show a nonlinear behavior, and the fit will predict an incorrect value for the dark count at $t_{0}$. Pixels of this type will have a decreasing slope in a plot of dark count versus exposure time (see Fig. 5.1b) and the model will give an actual dark count that is lower than predicted by the fit at the lower exposure times.

To evaluate the difference between the dark count obtained from the linear fit, $D_{f i t}$, and the dark count calculated at the longer exposure time, $D$, we define:

$$
\Delta D=D_{f i t}\left(t_{0}\right)-D\left(t_{0}\right)=m t_{0}-D\left(t_{0}\right)
$$

Using the parameters given in Table 5.1, we obtain a graph of $\Delta D$ versus $m$ for the entire set of modeled pixels when evaluated at $t_{0}=1500 \mathrm{~s}$ as seen in Fig. 5.4. The $\Delta D$ versus $m$ plane is divided into intervals of size $250 \mathrm{DN}$ by $0.5 \mathrm{DN} / \mathrm{s}$ and each pixel with a value falling within a given interval corresponds to an increase of one unit at that interval. The shades of gray show how many pixels were in a specific interval. Because of the wide range of frequencies, the gray scale is represented logarithmically. 


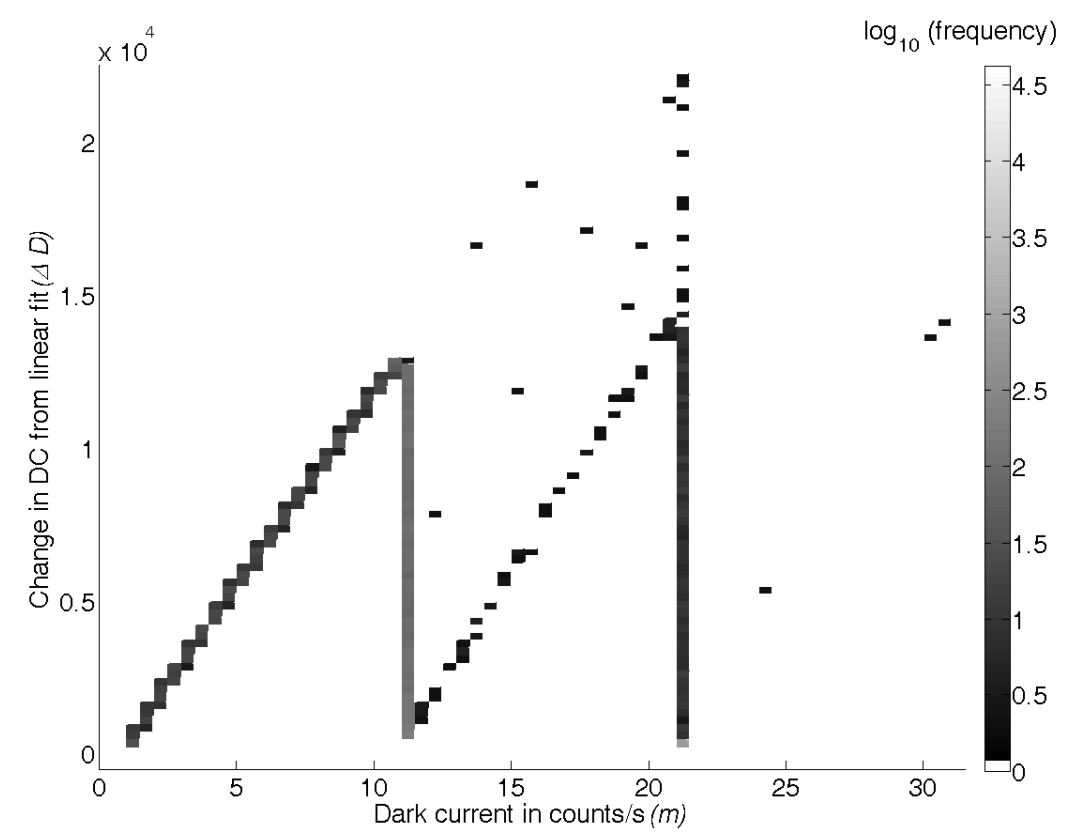

Fig. 5.4- Modeled dark current for $1 \times 10^{6}$ pixels from a linear fit over the first $50 \mathrm{~s}$ versus the change in dark count, $\Delta D$, evaluated at $1500 \mathrm{~s}$

Figure 5.5 shows a schematic representation of some of the various types of pixels examined by the model. For a pixel with a single impurity that does not leave the collection area during the first $50 \mathrm{~s}$, as illustrated by Pixel A in Fig. 5.5, the dark current will be $11 \mathrm{DN} / \mathrm{s}$. If additionally the impurity stays fully within the collection area at the longer exposure time (1500 s in the case of Fig 5.4) $D$ will be equal to $D_{f i t}$ and $\Delta D$ will be zero. If the impurity does not remain in the depletion region at the longer exposure time, such as Pixel $\mathrm{B}$, the pixel will have a $D$ less than the predicted $D_{f i t}$ and $\Delta D$ will be non-zero. Therefore, the pixel will lie in the grouping parallel with the y-axis. 

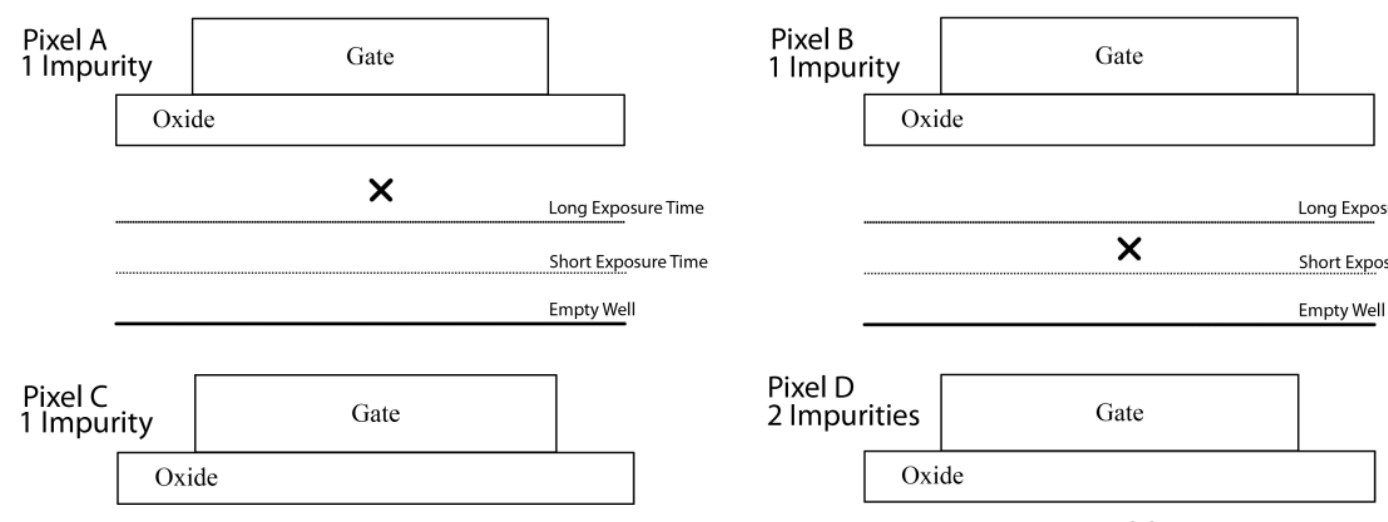

Long Exposure Time
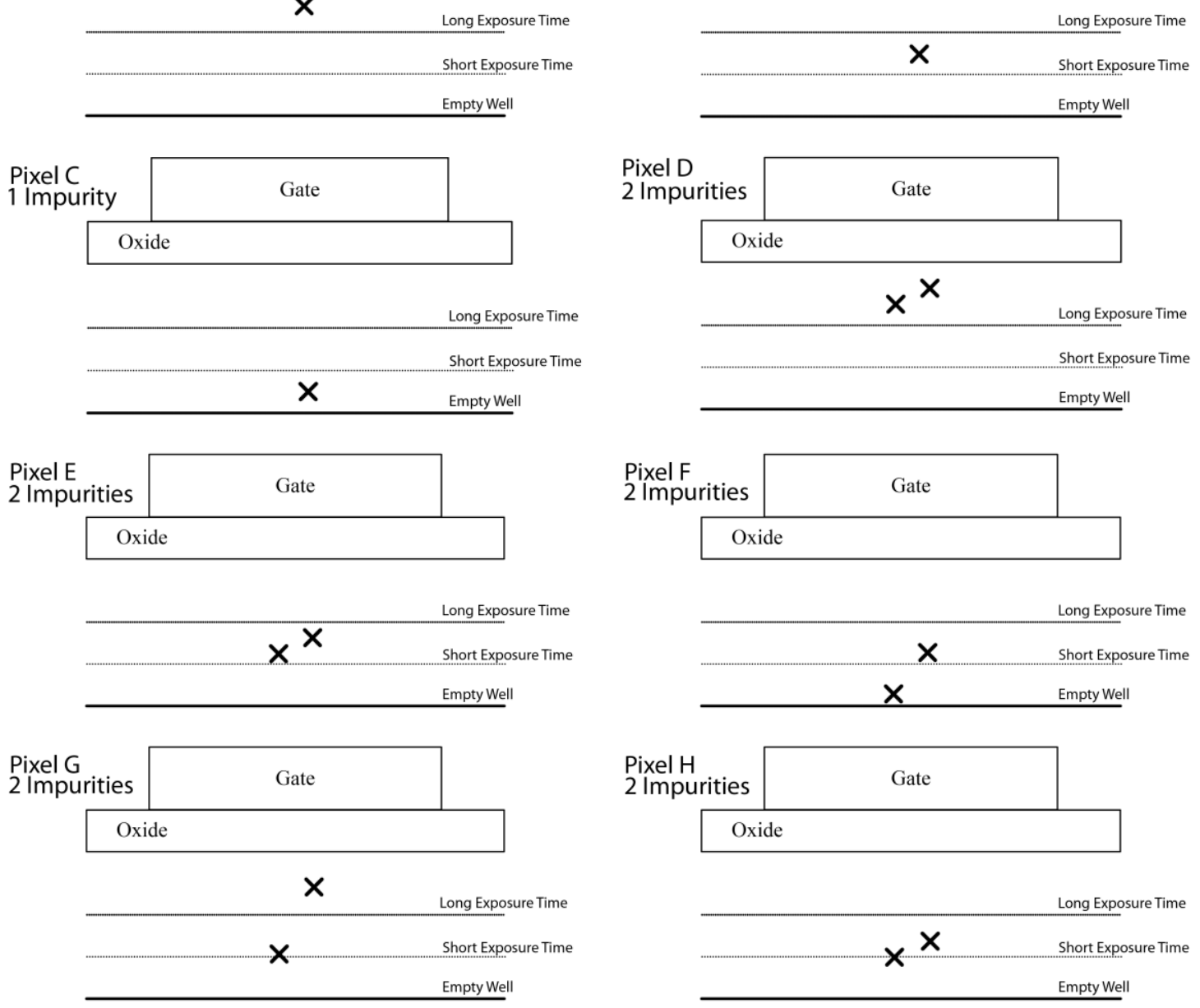

Fig. 5.5- Schematic diagrams of possible cases for depths of impurities in modeled pixels

However, if the impurity does not stay within the depletion region for the collection times where the linear fit is evaluated, the dark current will be less than $11 \mathrm{DN} / \mathrm{s}$. The impurity will therefore not stay within the depletion region at the longer exposure time. In this case, $D$ will be about zero and the pixel, similar to Pixel $\mathrm{C}$, will lie within the slanted linear grouping of pixels in Fig. 5.4 that starts at the origin of the graph. 
A pixel with two impurities that do not leave the collection area during the first $50 \mathrm{~s}$ will have a dark current of $21 \mathrm{DN} / \mathrm{s}$. In the case where the impurities are also fully within the collection area for the longer exposure time, as seen for Pixel D in Fig. 5.4, the extrapolated fit will agree with the value for $D$ and therefore $\Delta D$ will be zero. If either or both impurities leave the collection region during the longer exposure time, such as Pixel E, the pixel will have a smaller $D$ than $D_{f i t}$ value and thus have a nonzero $\Delta D$ value and lie in the group parallel to the $\mathrm{y}$-axis at about $21 \mathrm{DN} / \mathrm{s}$. In the case where one or both impurities do not stay within the collection region during the times used by the linear fit (Pixel F is one example of this type), the dark current for these pixels will be less than $21 \mathrm{DN} / \mathrm{s}$. If only one impurity leaves the collection region during these times, the dark current will stay between 11 and $21 \mathrm{DN} / \mathrm{s}$, whereas if both leave, the dark current can produce less than $11 \mathrm{DN} / \mathrm{s}$. The impurities will also leave the collection region during the longer exposure time and will therefore lie in the slanted regions of the graph. If one impurity stays within the region for the entire longer collection time while one is quickly removed from the region, similar to Pixel G, the pixel will lie in the diagonal region crossing the axis at about $11 \mathrm{DN} / \mathrm{s}$. If during the longer collection time one impurity produces some dark current before being removed from the collection region while the other is quickly removed, such as Pixel $\mathrm{H}$ would, the pixel will lie between the two diagonal regions.

From Eq. 5.24, pixels with three or more impurities of this type are possible but uncommon. A few pixels with three impurities are displayed in Figs. 5.4 and 5.8, but for simplicity sake not discussed in further detail here. 
The varying degrees of the nonlinear response to exposure time can be more clearly seen in a graph similar to Fig. 5.1 for the modeled pixels. Seen in Fig. 5.6 are DN values due to dark signal in response to exposure time for modeled pixels of the types given in Fig. 5.5. Pixel A has an $m$ value of $11 \mathrm{DN} / \mathrm{s}$ and $\Delta D$ of zero. The extrapolated value from a linear fit would be accurate for any exposure time for this pixel due to the impurity staying fully within the depletion region and therefore the pixel having a constant generation rate up to saturation. Similarly, as can be seen in Fig. 5.6, a pixel containing two impurities that stay fully within the depletion region for all exposure times, such as Pixel D, has a consistent rate of dark current but a greater slope of 21 DN/s. The remaining pixels shown in Fig. 5.6 all have a nonlinear dark count with respect to exposure time and a linear fit at low exposure times will lead to an overestimation of dark counts when extrapolated out to longer exposure times. Pixel types $\mathrm{G}$ and $\mathrm{H}$ were left off this graph due to having values for the displayed ranges too similar to those of A and B respectively. Of course, these pixels will have different responses at values less than or greater than those displayed. 

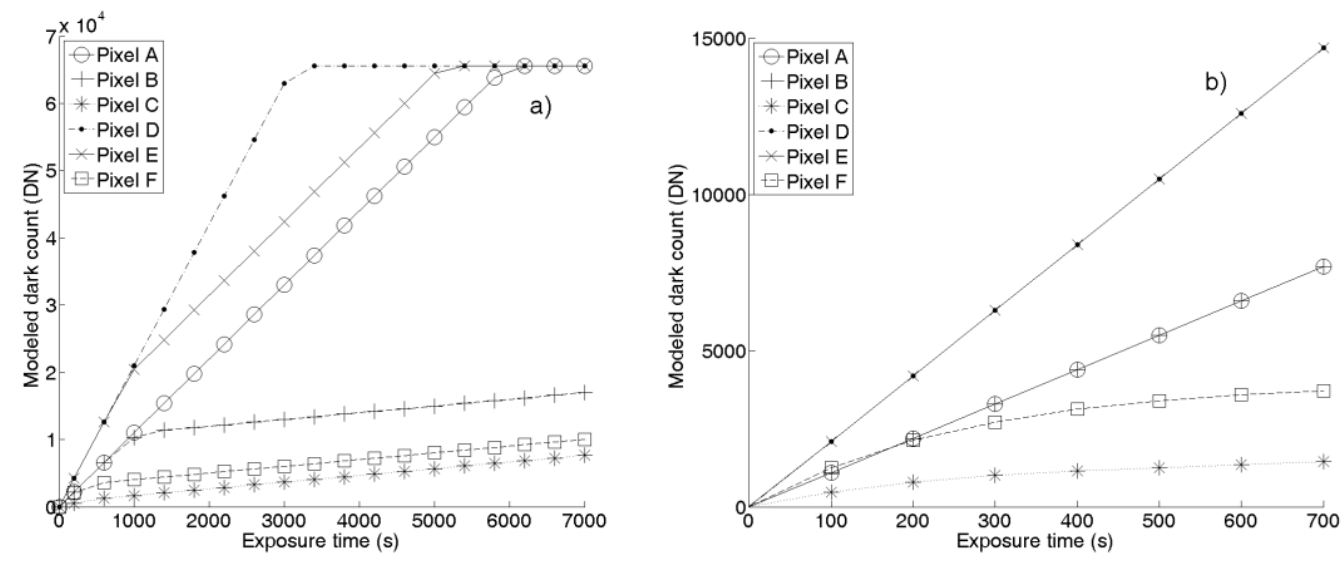

Fig. 5.6: Dark count as a function of exposure time for 8 pixels modeled using the parameters given in Table 1. (a) Exposure times up to 7000 s. (b) Enlargement with exposure times up to $700 \mathrm{~s}$

\subsection{Discussion}

The observed results from the model correspond to many features seen in similar plots of experimental data for existing cameras. One such camera displaying this is the SBIG ST-8XE with a KAF-1602E CCD sensor. Chapter 3 included figures similar to Fig 5.4, where exposures were taken to highlight the effects discussed above. Using the settings listed in Table 1, except for changes detailed in this section, the model was used to generate graphs matching the actual experimental data. The KAF-1602E has $1.56 \times 10^{6}$ pixels as well as a gain of $2.3 \mathrm{e}-/ \mathrm{DN}$ and these values were used for $N_{p i x}$ and $g$ respectively. Based on dark current for a standard pixel with very little dark current production $R_{0}$ was taken to be $0.5 \mathrm{DN} / \mathrm{s}$ and based on dark frame histograms for the peak of hot pixels in the imager, we used $9.5 \mathrm{DN} / \mathrm{s}$ for $R_{l} . N_{\text {imp }}$ was chosen to be 20,000 impurities to generate approximately the same number of pixels as seen in the peak of pixels with about $10 \mathrm{DN} / \mathrm{s}$ for Fig. 5.7a. This figure represents the change in dark count between the experimental dark current values at an exposure time of $1800 \mathrm{~s}$ 
from the linear fit of data collected between 5 and 60s versus the rate of dark current generation, the slope of the linear fit, at those shorter exposure times. The previous publications included groupings that were boxed and labeled for the ease of discussion and we have left the boxes in these figure, as well as superimposed them in the modeled data, for comparison. Figures $5.7 \mathrm{~b}$ and $5.7 \mathrm{c}$ used modeled data at the same exposure times as were taken to create the graph in Fig. 5.7a.

While most of the variables were chosen to correspond with typical values understood to be common in sensors, the value chosen for $\delta$ for both Figs. 5.4 and $5.7 \mathrm{c}$ were picked to correspond with features seen in empirical data for both the KAF-1602E and another imager, a Kodak KAF-0400 CCD chip in a Meade Pictor 416XT camera. A $\delta$ value of $3.0 \times 10^{-6} \mathrm{~cm}$ provided for the best fit for the groupings and a run with this value is displayed in Fig. 5.7c. To highlight the potential effect a variation of the $\delta$ value will have, the $\delta$ value in Fig. $5.7 \mathrm{~b}$ is $3.0 \times 10^{-5} \mathrm{~cm}$, an order of magnitude larger than the value used for Fig. 5.7c. All other variables were kept constant between the two sets of data. Two effects from a larger $\delta$ value combine to decrease the change in the dark current between the extrapolated linear fit and the amount of dark current collected at long exposure times. The first effect occurs when the impurity is within the collection well at short exposure times and results in a decrease in slope of the linear fit from the loss of generated electrons. The second effect is an increase in collection of generated electrons at longer exposure times when the impurity is not within the collection well. This shows the potential effect a variation of the $\delta$ value will have. 

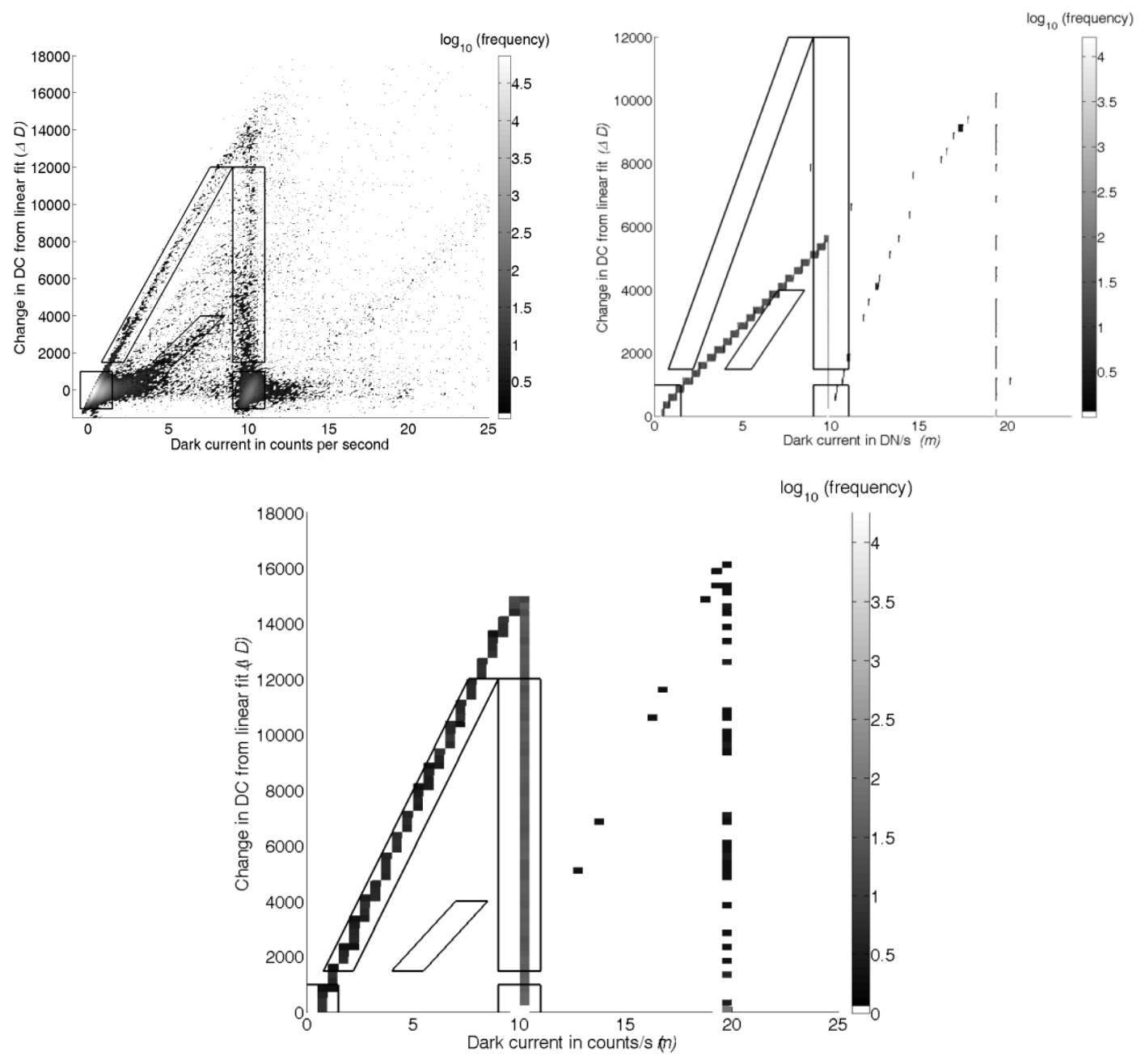

Fig. 5.7: (a) Dark current from linear fit versus the change in dark count, $\Delta \mathrm{D}$, for the KAF-1602E [6] evaluated at $1800 \mathrm{~s}$, (b) modeled data resulting in a poor fit due to using a $\delta$ value of $3.0 \times 10^{-5} \mathrm{~cm}$, (c) modeled data with a good fit using a delta value of $3.0 \times 10^{-6} \mathrm{~cm}$.

For many of the features, there is agreement between experimental and modeled data. In particular, the major groupings of pixels seen at about $10 \mathrm{DN} / \mathrm{s}$ in Fig. 5.7a, those seen in the horizontal groupings above these values, and those seen at a diagonal connected to the top of those horizontal groupings, are all present in the experimental as well as the modeled data. All of these pixels can therefore be explained by a pixel 
having a particular type of impurity that generates dark current at a rate of about 9.5 DN/s. The location of the pixel in the graph will depend on what the depth of the impurity is compared to the quantity of signal charge accumulated in the various exposures used to create the graphs. In addition, there is evidence in the experimental data of groupings seen due to pixels with two impurities including the extension of the diagonal grouping past the horizontal grouping at about $10 \mathrm{DN} / \mathrm{s}$.

While not sufficient to explain all of the features seen in the experimental data, such as the groups of pixels off the diagonal and the number of pixels seen in each of these groupings, many of the features are captured by the model. The deficiencies are likely due to the model being essentially only one-dimensional whereas a more complete model would require analyzing the change in the shape of the three-dimensional depletion edge. In addition, our model assumes a uniform distribution of impurities as a function of depth within the pixel, however there may be processes that result in a more systematic location of impurities. A larger percentage of impurities within the region of depletion edge variation will result in a greater number of pixels in the labeled groups in the experimental data. In addition, a three-dimensional model in which the depletion edge moves significantly more near the edges of the pixel may result in more pixels affected by a similar type of nonlinearity. One possible explanation for more impurities in this region of greater change, would be impurities introduced in building the channel stops. Inclusion of these considerations is an area for future work. 


\subsection{Further effects of variations in model parameters}

Table 5.1 lists the variables comprising the model used to reproduce dark current behavior in the Kodak imagers. Discussion regarding the model thus far has largely been focused on this reproduction, with the exception in the previous section discussing variations in the $\delta$ size, however, further varying the parameters allows for additional insight into pixels with such impurities. For instance, increasing the total number of impurities distributed across the modeled imager, $N_{\text {imp }}$, allows for observing a more complete overview of pixels with different impurity locations especially for pixels with two or more impurities. For instance, Fig. 5.8 highlights this possibility with 10,000 impurities in Fig. 5.8a and 100,000 impurities in Fig. 5.8b. All other parameters for these plots coincide with values given in Table 5.1 and can be compared to the previous plots with the value for the number of pixels fitting the KAF-1602E sensor of 50,000 impurities. Little to no detail is offered by Fig. 5.8a for pixels with two or three impurities, where as the myriad of possibilities for variations in impurity depths are observed in Fig. 5.8b. This can especially be noted for pixels with dark current in count/s greater than 20 and a $\Delta D$ greater than 15,000 . 

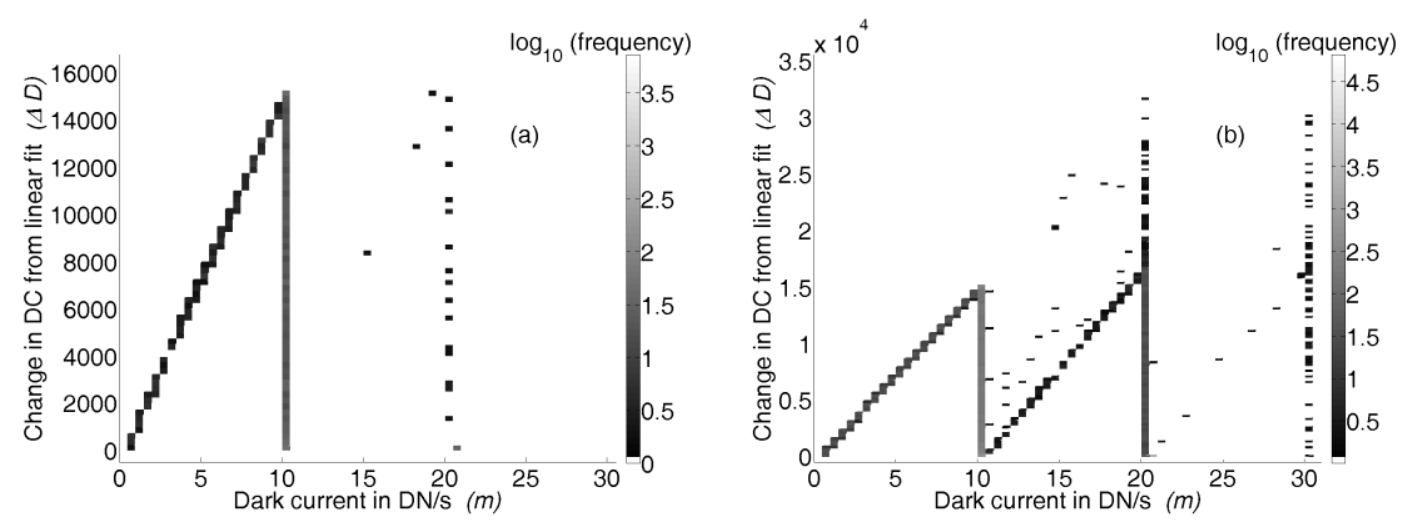

Fig. 5.8: Dark current from linear fit versus the change in dark count, $\Delta D$, modeled with (a) 10,000 impurities, and (b) 100,000 impurities.

A potential area of future research will involve varying the gate voltage using a customizable camera system built by our lab. By varying the gate voltage, $V_{g}$, the locations of the depletion region and depletion edge are also changed. Therefore, an impurity previously near the depletion edge, say with no signal charge present and using $-5 \mathrm{~V}$ as the SBIG camera system did, could be made to be either fully within the depletion region, resulting in a linear hot pixel, or completely outside the depletion region, resulting in a typical non-hot pixel, just by varying the gate voltage. As the gate voltage is set universally for all pixels across the imager in a $\mathrm{CCD}$, a change in the pattern of pixel locations in a graph of the dark current from linear fit versus the change in dark count would be observed. One such possibility is presented in Fig. 5.9 with a gate voltage set to $-5 \mathrm{~V}$, as used previously, shown in Fig. 5.9a and a gate voltage of $0 \mathrm{~V}$ shown in Fig. 5.9b. A noticeable change in the pixel behavior is observed for pixels with small dark current values at low exposure times and, universally, $\Delta D$ values are smaller in Fig. 5.9b. It is our hope that the model and 
experimental results would yield similar changes to further validate the model and present possible avenues for evaluating and designing optimal imagers.
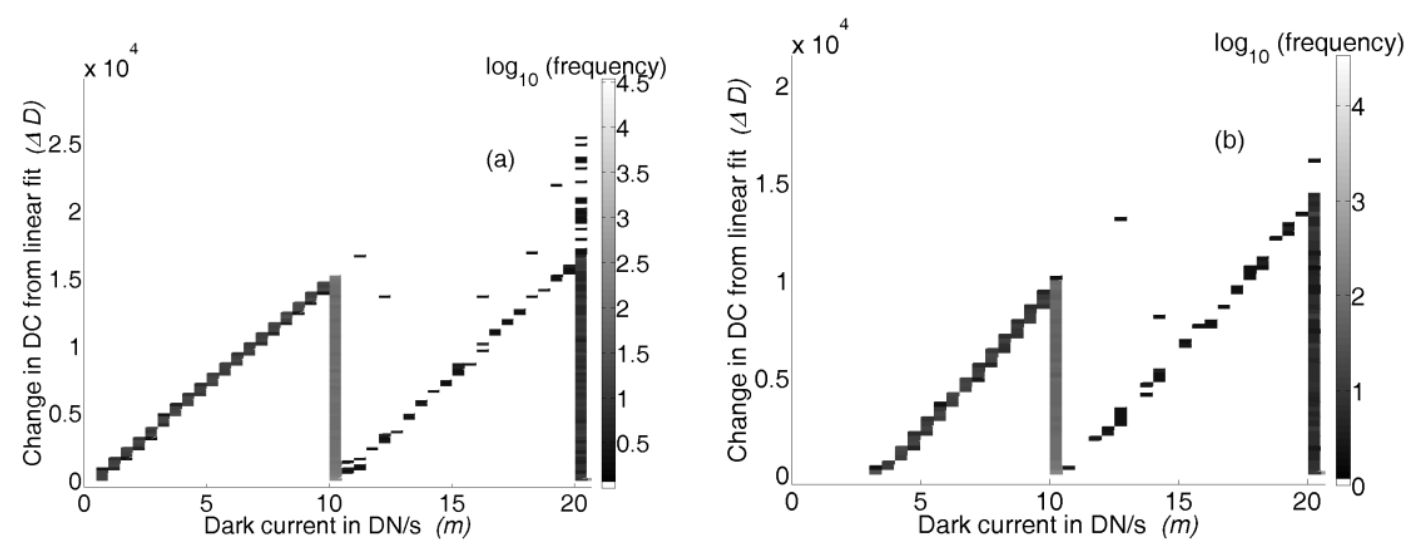

Fig. 5.9: Dark current from linear fit versus the change in dark count, $\Delta D$, modeled with a gate voltage of (a) $-5 \mathrm{~V}$, and (b) $0 \mathrm{~V}$.

\subsection{Conclusion}

A model utilizing a dynamic depletion edge, variable depths of impurities, and number of impurities within digital imager pixels has been introduced. The model has shown to predict the characteristic behavior of dark current including nonlinear behavior with respect to integration time. The model captures the essential aspects observed in the experimental data. The model, in conjunction with the correct parameters of the imagers along with experimental data, makes it possible to reveal previously unknown information about the pixels including depths of certain types of impurities. 


\section{CHAPTER 6 - MODELING OF DARK CURRENT RESPONSE TO ILLUMINATION}

\subsection{Introduction}

In a pixel within a digital imager, generally either a $\mathrm{CCD}$ or CMOS device, appropriate doping of the semiconductor substrate and application of gate voltages create a region free of mobile carriers. This region is referred to as the the depletion region or the well. This region fills with charge after incoming photons are absorbed or thermal energy elevates a charge from the valence to the conduction energy band. As the signal charge, in the form of photoelectrons and/or dark current, fills the depletion region, the electric field generating that region is altered, and the size of the region is reduced. We present a model that describes how this dynamic depletion region, along with the location of impurities, will result in pixels that produce less dark current after being exposed to light. Additionally, we show nonlinear production rates with respect to exposure time are a result of this model. These types of effects have been observed in digital imagers allowing us to compare empirical data with the modeled data. This section further shows how the model is able to replicate empirical results and is based on material presented in [31].

The amount of dark current is generally expected to be about the same regardless of whether the imager is exposed to light or left in the dark. However, it was found in prior studies, as discussed in Chapter 2, that the dark current generation rate for certain pixels was changed by the presence of signal charge in the well [17]. For these pixels, the standard method of removing the dark current from the image is insufficient. In 
particular, after an image is taken, the standard method of correction involves taking an exposure at the same integration time as the image except now leaving the shutter closed. This "image" is referred to as a dark frame and is employed to remove the dark noise present in the original image. The pixel values from this dark frame are subtracted from the original image pixel-by-pixel. While more complex methods of correction exist, such as taking multiple dark frames and averaging them to reduce shot noise, they generally all rely on the expectation that the dark current will be consistent when the shutter is open or closed.

Using the mechanisms described in the previous chapter, we present how a moving depletion region in a pixel can duplicate changes to dark current generation rate in empirical data from imagers when exposed to light compared to not being exposed. The model of this effect assumes the presence of one or more impurities in the pixel that produce a distinctive rate of dark current per impurity. Due to filling with signal charge, the depletion edge of the potential well will move, possibly far enough to shift the impurity out of the well. Minimal amounts of dark current will therefore be collected from this impurity while no longer in the well. Light exposure essentially results in large amounts of signal charge being collected by the well and rapidly shifts the depletion edge. Therefore, it is possible for a pixel with one or more impurities in the region of the depletion edge shift to generate significantly less dark current when exposed to light than when not exposed. 
Similarly, although generally slower, thermal excitation of signal charges collected by the well also results in a shrinking depletion region. For pixels with impurities near the depletion edge, the collection rate of dark current from these impurities will decrease with increasing exposure time. The model's ability to duplicate features seen in empirical data due to the thermal excitation versus exposure time has been described in detail in the previous chapter. However, we present here how these behaviors can be modeled using similar parameters as the response to illumination, and similarities will be compared between the two.

\subsection{Dynamic depletion edge model}

\subsubsection{Model overview}

The modeled pixel consists of a one-dimensional depletion region with one or more impurities within the region. Figure 6.1 shows a two-dimensional representation of the one-dimensional model. As the depletion well fills with charge, the edge of the depletion region, $\omega$, contracts and moves closer to the oxide layer. As it contracts, the impurity sits closer to the edge and, depending on the location, will ultimately no longer be located within the depletion region. When this occurs, the pixel will no longer collect the majority of the thermally generated electrons from the impurity. We have added a region, defined by plus or minus $\delta$ from the depletion edge, where there is a probability that either thermally generated electrons will escape the depletion region when the impurity sits within the depletion region, or a probability that thermally generated electrons will be collected by the depletion well when the impurity sits just outside the depletion edge. 


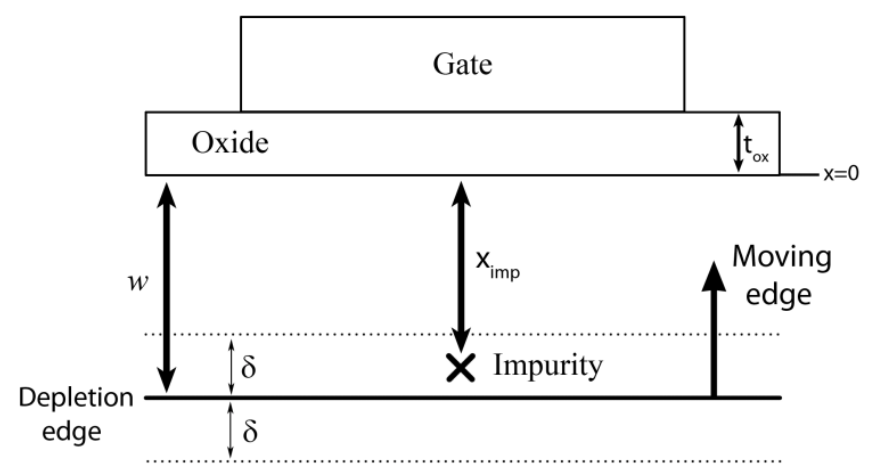

Fig. 6.1- Two-dimensional representation of the one-dimensional pixel with an impurity near the moving depletion edge

We have previously allowed charge to be accumulated slowly to model pixels' dark current response to gradual collection of dark signal, simulating a relatively long exposure dark-frame as discussed in Chapter 5. Now, we present data where we allow the pixels to not only gradually accumulate signal charge during a relatively longexposure dark frame, but additionally provide a large initial signal charge to simulate an exposure to a flash of light during the beginning of the integration time. These conditions simulate a study where dark frames were taken both after a large flash of light and without the flash of light as described in Chapter 3. Integration time of the dark frame as well as initial signal charge are variables that can be chosen by the user, whereas the rest of the values were chosen to be consistent with representative values found for typical imagers. The parameters used by the model are summarized in Table 6.1. When using these values, the depletion edge starts at a distance of $5.33 \mu \mathrm{m}$ from the oxide layer and moves to $3.93 \mu \mathrm{m}$ when the depletion region is entirely filled with signal charge. 


\begin{tabular}{|c|c|c|}
\hline Name & Parameter & Value Chosen \\
\hline Gate voltage & $V_{g}$ & $-5 \mathrm{~V}$ \\
\hline Buried channel implant dose & $Q_{c d}$ & $2.5 \times 10^{12} \mathrm{~cm}^{-2}$ \\
\hline Oxide thickness & $t_{o x}$ & $7.2 \times 10^{-6} \mathrm{~cm}$ \\
\hline $\begin{array}{c}\text { Number of acceptors per } \\
\text { volume }\end{array}$ & $N_{a}$ & $5.0 \times 10^{14} \mathrm{~cm}^{-2}$ \\
\hline Relative oxide permittivity & $\varepsilon_{O x} / \varepsilon_{0}$ & 3.9 \\
\hline $\begin{array}{l}\text { Relative semiconductor } \\
\text { permittivity }\end{array}$ & $\varepsilon_{s i} / \varepsilon_{0}$ & 11.7 \\
\hline Range & $r$ & $1.0 \times 10^{-5} \mathrm{~cm}$ \\
\hline Straggle & $\sigma$ & $3.0 \times 10^{-5} \mathrm{~cm}$ \\
\hline Camera gain & $g$ & $3.0 \mathrm{e}-/ \mathrm{DN}$ \\
\hline $\begin{array}{l}\text { Rate of dark current without } \\
\text { impurity }\end{array}$ & $R_{0}$ & $1.0 \mathrm{DN} / \mathrm{s}$ \\
\hline $\begin{array}{l}\text { Rate of additional dark } \\
\text { current due to a single } \\
\text { impurity }\end{array}$ & $R_{1}$ & $10.0 \mathrm{DN} / \mathrm{s}$ \\
\hline Pixel collection area & $A$ & $2.1 \times 10^{-7} \mathrm{~cm}^{2}$ \\
\hline Total pixels & $N_{p i x}$ & $1 \times 10^{6}$ \\
\hline Full well count & & 65,536 DN (16 bit) \\
\hline Number of impurities & $N_{i m p}$ & 50,000 impurities \\
\hline Delta edge & $\delta$ & $5.0 \times 10^{-6} \mathrm{~cm}$ \\
\hline Integration time & & $500 \mathrm{~s}$ \\
\hline Initial signal charge & & 30,000 counts \\
\hline
\end{tabular}

Table 6.1- Modeling parameters

\subsubsection{Modeled response to illumination}

To get an overview of the effect of illumination, or an initial partial filling of the potential well, we define $\Delta D_{\text {Light }}$ :

$$
\Delta D_{\text {Light }}=D_{N L}-D_{L},
$$

where $D_{N L}$ is the total number of dark counts generated with no initial signal charge in the potential well for a given integration time, and $D_{L}$ is the total number of dark counts generated for the same integration time after the initial signal charge is added. 
In practice, this initial signal charge could be added by exposing the imager to an initial flash of light prior to the longer exposure time where only dark current is collected.

A two-dimensional distribution of the loss in dark count, $\Delta D_{\text {Light }}$, versus the dark-count level, $D_{N L}$, was created using this model. The $\Delta D_{\text {Light }}$ versus $D_{N L}$ plane was divided into intervals of size $250 \times 250 \mathrm{DN}$, and the shades of gray represent the logarithm of the number of pixels in a specific interval. Figure 6.2 represents this distribution for modeled data using the parameters given in Table 6.1. Varying the parameters, such as DC generation rate, or initial signal charge will result in variations in the data and the graph as presented in Chapters 2, 3, and 6.

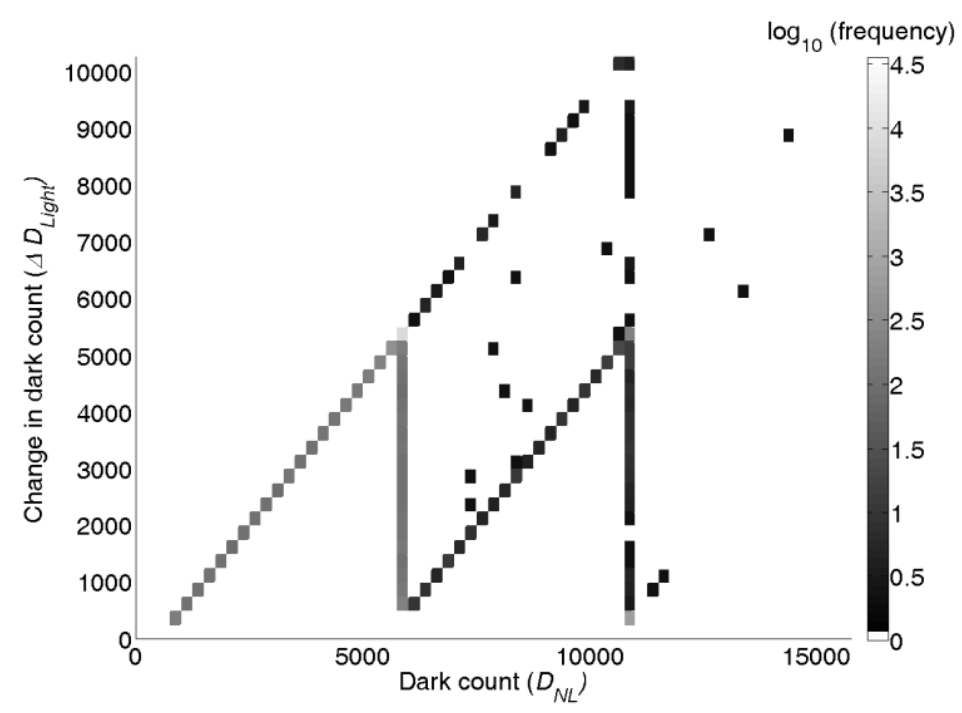

Fig. 6.2- Change of dark count after illumination versus dark count of a $500 \mathrm{~s}$ dark frame with an initial count of 30,000. 
A pixel with a single impurity that remains fully within the depletion region for the entire collection time without exposure to light will have a $D_{N L}$ of about 5,500 DN. If additionally the impurity remains fully within the depletion region during the collection time after the initial flash, the impurity will produce just as much dark current and will have a $\Delta D_{\text {Light }}$ value of about 0 . However, if the initial flash of light moves the depletion edge past the impurity, the pixel will collect no dark current from the impurity, and the pixel will have a $\Delta D_{\text {Light }}$ of about 5,500. If the depletion edge moves past the impurity at some point during the collection time after the exposure of light, the pixel will lie in the grouping of pixels parallel to the y-axis at about 5,500 DN. Now, if the impurity lies close enough to the depletion edge such that it is partially or fully removed from the depletion region during the collection time without exposure to light, the pixel will have a $D_{N L}$ value less than $5,500 \mathrm{DN}$. Of course, the impurity will also be removed from the depletion region by the exposure to light or during the subsequent collection time after the exposure, so the pixel will have a small or zero $D_{L}$ value, and $\Delta D_{\text {Light }}$ will be about the same value as $D_{N L}$.

A pixel with two impurities, which remain fully within the depletion region during the collection time with no exposure to light, will have a $D_{N L}$ of about $11,000 \mathrm{DN}$. If both those impurities are also fully within the depletion region for the entire collection time after the flash, the pixel will again produce just as much dark current and will have a $\Delta D_{\text {Light }}$ value of about 0 . If both impurities are fully removed from the depletion region by the flash, the pixel will produce no dark current for the collection period giving a $D_{L}$ value of 0 , and $\Delta D_{\text {Light }}$ will also be about $11,000 \mathrm{DN}$. If just one impurity is 
removed from the collection region by the flash while the other remains fully within the region for the entire collection time, the $D_{L}$ value will be about 5,500 and thus the $\Delta D_{\text {Light }}$ value will also be about 5,500 . If one or both impurities are removed from the collection region during the collection time after the flash, the pixel will lie in the grouping parallel to the y-axis. For the cases where one or both impurities are partially removed from the collection region during the collection time with no exposure to light, $D_{N L}$ will have values less than $11,000 \mathrm{DN}$. During or after the flash, if one impurity stays fully within the collection region while the other is removed, $D_{L}$ will have a value between 5,500 and 11,000 , and therefore $\Delta D_{\text {Light }}$ will be between 0 and 5,500, approximately the same as $D_{N L}$ for that pixel. If both pixels are removed from the depletion region during or after the flash, $D_{L}$ will be between 0 and 5,500. If both impurities are turned off during the flash, $\Delta D_{\text {Light }}$ will be approximately the same as $D_{N L}$. However, if one of the impurities is removed from the depletion region only in the collection time after the flash, the pixel will lie between the two diagonal lines with a $D_{N L}$ value between 5,500 and 11,000 .

Additionally, with the total number of impurities we use in the model, individual pixels with more impurities of this type are possible but become statistically unlikely. There are a few pixels with three impurities which can be seen in Fig. 6.2 with $D_{N L}$ values greater than 11,000 . 


\subsubsection{Modeled nonlinear behavior}

Pixels are typically not only expected to have a linear response to light but also to dark current generated versus time. As such, to get an idea of the linear behavior of the pixels, a linear fit is calculated for dark current generation rates at low exposure times:

$$
D_{\text {fit }}=m t
$$

where $t$ is the time, and $m$ is the slope of the fit at low exposure values. To generate Fig. 6.3, we have used the model to generate dark noise for exposure time values from 5 to $50 \mathrm{~s}$ and performed a linear fit on these exposure times. This linear fit can be extrapolated out to arbitrarily longer exposure times, $t_{0}$, to calculate the difference, $\Delta D$, between the predicted generated dark noise, $D_{f i t}\left(t_{0}\right)$ compared to the actual modeled noise, $D\left(t_{0}\right)$ :

$$
\Delta D=D_{f i t}\left(t_{0}\right)-D\left(t_{0}\right)=m t_{0}-D\left(t_{0}\right)
$$

Figure 6.3 is representative of the graphs generated using this method and using the values for the parameters given in Table 6.1. Additionally, $t_{0}$ is chosen to be $1800 \mathrm{~s}$. The $\mathrm{x}$-axis is now the slope, $m$, and the $\mathrm{y}$-axis is $\Delta D$. 


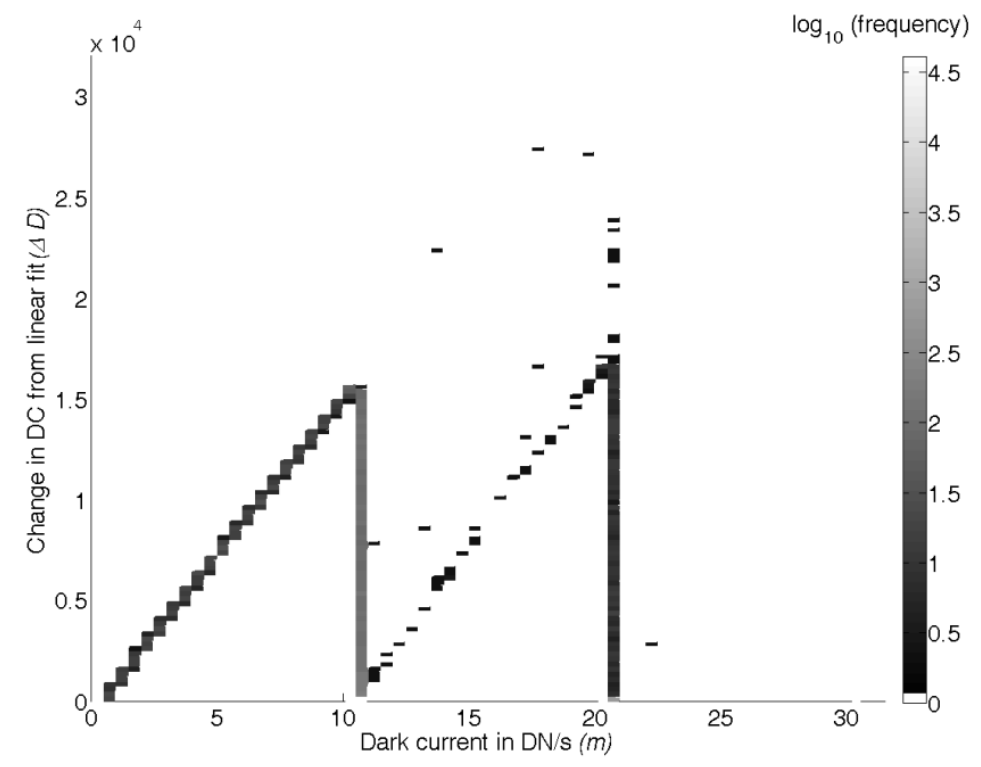

Fig. 6.3- Modeled dark current for $1 \times 10^{6}$ pixels from a linear fit over the first $50 \mathrm{~s}$ versus the change in dark count, $\Delta D$, evaluated at $1800 \mathrm{~s}$.

Figure 6.3 appears to share general features with the graphs generated with the simulated moving depletion edge due to an initial exposure to light. Discussion on the location of pixel impurities and where they would end up on the graph is similar to discussion in section 6.2.2 and discussed more in depth in the previous chapter.

\subsection{Discussion}

The observed results from the model correspond to many features seen in similar plots of experimental data for existing cameras. Two cameras displaying these behaviors are the SBIG ST-8XE with a KAF-1602E CCD sensor and a Meade Pictor 416XT with a Kodak KAF-0400 CCD chip. Chapter 2 included figures similar to those in Fig 6.2, where exposures were taken to highlight the effects discussed above. Using the settings listed in Table 6.1, except as noted in this paragraph, the model was used to 
generate graphs matching the actual experimental data. The KAF-1602E has $1.56 \times 10^{6}$ pixels and a gain of $2.3 \mathrm{e}^{-/} \mathrm{DN}$, while the KAF-0400 has $3.93 \times 10^{5}$ pixels and a smaller gain of $1.2 \mathrm{e}^{-/} \mathrm{DN}$; these values were used for $N_{\text {pix }}$ and $g$ respectively. Data were taken at $288 \mathrm{~K}$ for the KAF-1602E and at $278 \mathrm{~K}$ for the KAF-0400. Based on dark current production for a standard pixel with very small dark current production at these temperatures, $R_{0}$ was taken to be $0.5 \mathrm{DN} / \mathrm{s}$ for both imagers, and based on dark-frame histograms for the peak of hot pixels in the imager, we used $9.5 \mathrm{DN} / \mathrm{s}$ for $R_{1}$ for the KAF-1602E and 9.0 DN/s for the KAF-0400. $N_{\text {imp }}$ was chosen to be 20,000 impurities for the KAF-1602E and 50,000 impurities for the KAF-0400. These values were chosen to generate approximately the same number of pixels as seen in the peak of pixels at about 6,000 DN in Fig 6.4a and 6.5a respectively. A $\delta$ value of $3.0 \times 10^{-6} \mathrm{~cm}$ provided for the best fit for the data groupings for the KAF-1602E, and a value of $5.0 \times 10^{-6} \mathrm{~cm}$ provided for the best fit for the groupings for the KAF-0400. This value is empirically chosen for the two imagers, and our model does not attempt to explain the differing values; they may depend on such factors as the doping concentration. For the graphs from the previous publications, groupings were boxed and labeled for the ease of discussion, and we have left the boxes in these figures as well as superimposed them in the modeled data for comparison.

\subsubsection{KAF-1602E}

Shown in Fig. 6.4a is the change in dark count after illumination versus dark count of a $600 \mathrm{~s}$ dark frame after an exposure to light leading to an average value of $26,000 \mathrm{DN}$ 
across the imager last mentioned in Chapter 2. These values were chosen as the integration time and initial signal respectively for the modeled data seen in Fig. 6.4b.
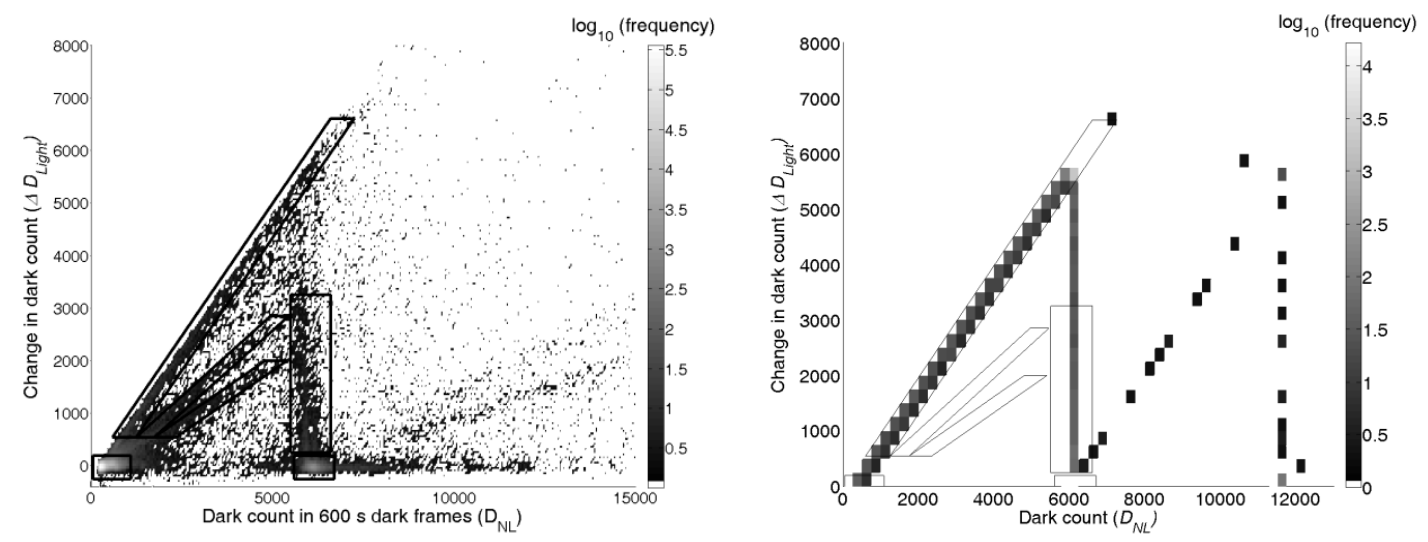

Fig. 6.4: Change of dark count after illumination versus dark count of a $600 \mathrm{~s}$ dark frame. (a) KAF-1602E for an average initial count due to the light exposure of approximately 26,000 at $288 \mathrm{~K}$ [17]. (b) modeled data.

For many of the features, there is agreement between experimental and modeled data. In particular, the major groupings of pixels seen at a dark count of about 6,000 in Fig. 6.4a, those seen in the vertical groupings above these values, and those seen at a diagonal connected to the top of those vertical groupings, are all present in the experimental as well as the modeled data. All of these pixels can therefore be explained by a pixel having a particular type of impurity that generates dark current at a rate of about 9.5 DN/s. The location of the pixel in the graphs will depend on what the depth of the impurity is compared to the quantity of signal charge accumulated in the various exposures used to create the graphs. In addition, there is evidence in the experimental data of groupings seen due to pixels with two impurities including the extension of the diagonal grouping past the vertical grouping at about $10 \mathrm{DN} / \mathrm{s}$. 
Experimental data using the SBIG camera used to generate graphs similar to Fig. 6.3 are not shown here; however, the model shared a similar success in predicting locations of groupings as seen in the graphs for the exposure to illumination in Fig. 6.4.

While not sufficient to explain all of the features seen in the experimental data, such as the groups of pixels off the main diagonals (with the greatest slope) and the specific number of pixels seen in each of these groupings, many of the features are captured. The deficiencies of the model are likely due to the model being essentially only onedimensional, whereas a more complete model would require analyzing the change in the shape of the three-dimensional depletion region. In addition, our model assumes a uniform distribution of impurities as a function of depth within the pixel; however, there may be processes that result in a more systematic location of impurities. A larger percentage of impurities within the region of the depletion edge variation will result in a greater number of pixels in the labeled groups in the experimental data. In addition, a three-dimensional model in which the depletion edge moves significantly more near the edges of the pixel may result in more pixels affected by a similar type of nonlinearity. One possible explanation for more impurities in this region of greater change would be impurities introduced in building the channel stops. Inclusion of these considerations is an area for future work. 


\subsubsection{KAF-0400}

Shown in Fig. 6.5a is a similar graph for the Meade imager system, where the change in dark count after illumination is plotted versus dark count of a $600 \mathrm{~s}$ dark frame after an exposure to light leading to an average value of $42,000 \mathrm{DN}$ across the imager. These values were chosen as the integration time and initial signal respectively for the modeled data seen in Fig. 6.5b.
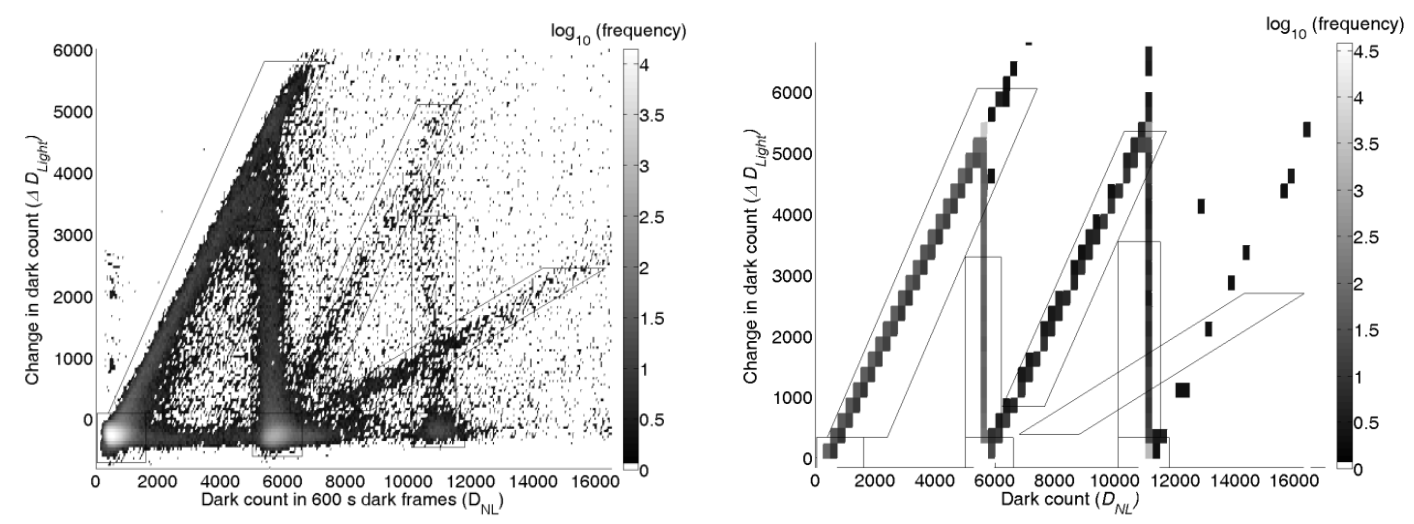

Fig. 6.5: Change of dark count after illumination versus dark count of a $600 \mathrm{~s}$ dark frame. (a) KAF-0400 for an average initial count due to the light exposure of approximately 42,000 at 278 K. [17] (b) modeled dark current.

Again, agreement in the features is seen in the graphs of the experimental and modeled data. Due to the relatively larger number of impurities seen in the KAF-0400, there is a second triangle of data seen with larger dark counts along the $\mathrm{x}$-axis. This feature is due to more pixels that have a higher likelihood of having two impurities. Thus, all of the features seen in Fig. 6.5b can be explained by a pixel having one or more impurities with a rate of production at 9.0 DN/s. There exists a grouping starting at about 6,000 counts and $0 \Delta D_{\text {Light }}$ that extends diagonally in the graph to 18,000 counts 
and a $\Delta D_{\text {Light }}$ of about 3,000 that is not explained by the model. This grouping shares similarities with the unexplained groupings in the KAF-1602E imager.

\subsection{Further effects of variations in model parameters}

Additional options for variations, other than those chosen to reproduce the behavior seen in the Kodak imagers are possible with the model. As observed in section 5.4, we can adjust the number of impurities to get obtain a more complete picture of the possible behavior of pixels with multiple impurities for nonlinear behavior with respect to exposure time. Using the same parameters in Table 6.1, but varying the number of impurities distributed across the imager, we observe that increasing the impurities from 10,000 in Fig. 6.6a to 100,000 in Fig. 6.6b, further elucidates the behavior of pixels with more than a single impurity. A larger spread of pixels are seen throughout the plot, with two and three impurity pixels populating more of the many possible states expected above a dark count of 6000 DN in Fig. 6.6b.
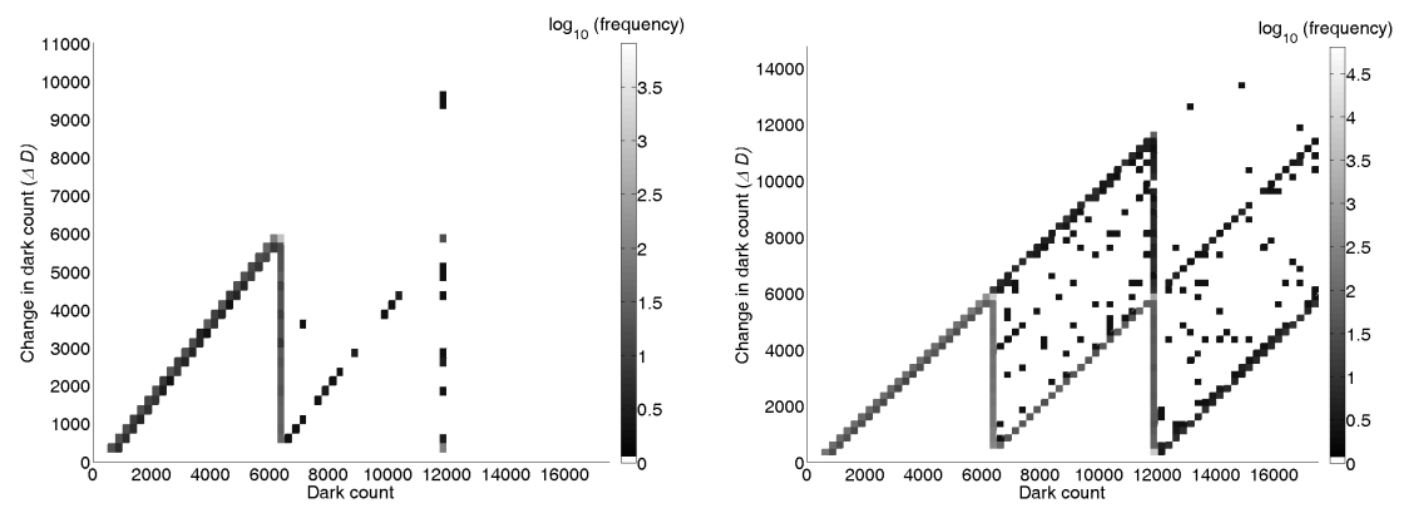

Fig. 6.6: Change of dark count after illumination versus dark count of a $500 \mathrm{~s}$ dark frame. (a) Modeled using 10,000 impurities 
distributed throughout the imager. (b) Modeled using 100,000 impurities distributed throughout the imager.

Further modifying the parameters could be used to validate the model while making similar changes in an experimental set-up as discussed in section 5.4. For instance, changing the gate voltage from $-5 \mathrm{~V}$ to $0 \mathrm{~V}$, while leaving all other parameters the same as listed in Table 6.1, significantly impacted the distribution in Fig. 5.8 and appears to decrease the distribution of possible states in Fig. 6.7 for pixels with multiple impurities. For instance, there appears to be no pixels with three impurities in Fig. $6.7 \mathrm{~b}$ with impurity distributions that place the pixel above $12,000 \mathrm{DN}$ and a $\triangle D$ of 6000 DN. Comparing these results to empirical data remains an exciting potential for future research.
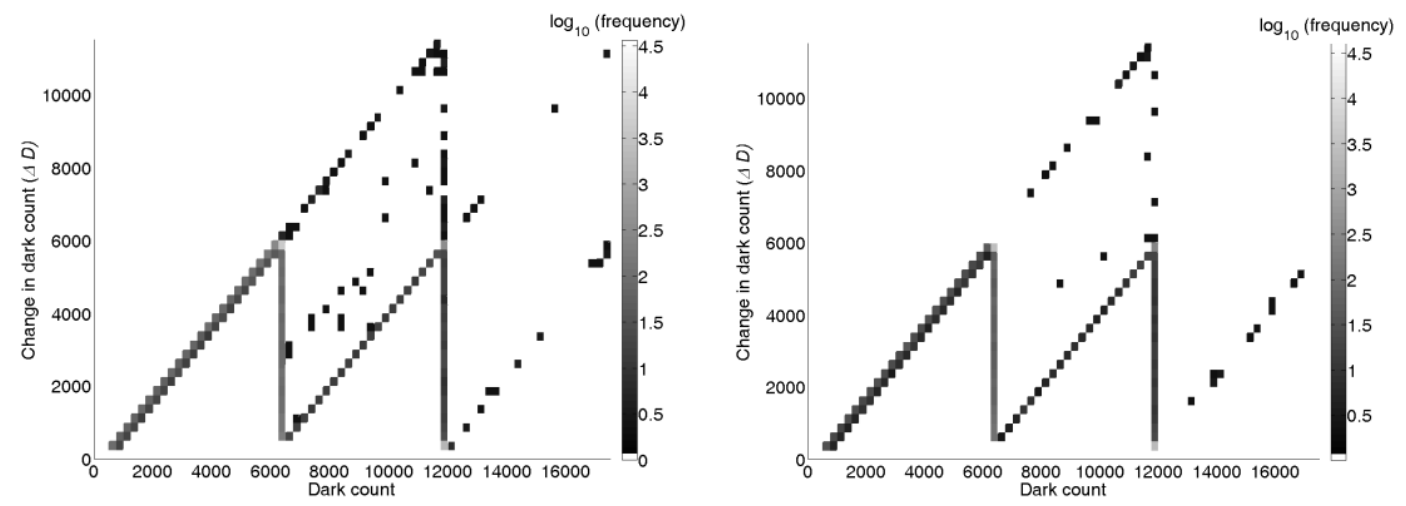

Fig. 6.7: Change of dark count after illumination versus dark count of a $500 \mathrm{~s}$ dark frame. Modeled dark current with a gate voltage of (a) $-5 \mathrm{~V}$ and (b) $0 \mathrm{~V}$.

\subsection{Conclusion}

Dark current generation rates that change with exposure to illumination compared to no exposure can be reproduced using a model of a one-dimensional depletion well with a moving depletion edge and containing one or more impurities near the edge. 
Additionally, the same behavior of a moving depletion edge is shown to be a mechanism of nonlinear collection rate of dark current. Control of the variables in the model allows for accurately predicting the amount of dark current generated by a pixel as the depletion well is filled with signal charge. The distinctive patterns and groupings seen in multiple camera systems when dark current generation rates are compared in the presence or absence of light are duplicated by this model. 


\section{CHAPTER 7- ACTIVATION ENERGY DEPENDENCY ON SIGNAL CHARGE IN CCD IMAGERS}

\subsection{Introduction}

In this chapter we present a study showing the dependency of calculated activation energies on signal charge in a CCD imager. Activation energies for some pixels are observed to vary between values at about half the bandgap of silicon calculated for low count levels to values approaching the bandgap when calculated at consistently high signal charge levels. As such, the traditional method of calculating activation energies using a single exposure time at varying temperatures will result in calculations where the pixel will obtain both high and low overall signal charge levels, resulting in a seemingly erratic value for the activation energy. Therefore, a method of calculating activation energies using a single signal charge, and thus count value, is proposed for obtaining more consistent results. Further, we demonstrate how a model of a fixed location impurity can lead to this behavior in pixels with a moving depletion edge due to a changing depletion region size.

A common method of analyzing temperature dependent phenomena is by using the Arrhenius equation:

$$
X=X_{0} \exp \left(-E_{A} / k T\right)
$$

to calculate the activation energy $E_{A}$. In this equation $X$ is a measured property, $X_{0}$ is the exponential prefactor, $k$ is Boltzmann's constant, and $T$ is the absolute temperature [13]. This empirical law was characterized in 1884 by J.H. van’t Hoff, but received its 
name from the Swedish chemist Svante Arrhenius who provided an explanation for the possible mechanism of the law in chemical reactions [14]. The law is often used to characterize chemical reactions that are temperature-dependent, as well as temperature dependent processes in solid state physics, including characterizing the temperature dependent dark current performance in digital imagers. The Arrhenius equation explicitly written to reference dark current generation as the measured property:

$$
D=D_{0} \exp \left(-E_{A} / k T\right)
$$

In this equation, $D$ is the generation rate of dark current and $D_{0}$ is the exponential prefactor.

The typical method of calculating dark current activation energies requires taking a series of dark frames over a range of temperatures at a particular exposure time. A plot of the natural $\log$ of counts versus the inverse of the temperatures, as seen in Figs. 7.1, is typically best characterized as a line. Such a plot, where the measured property is graphed versus the inverse of temperature, is referred to as an Arrhenius plot. From equation 7.2 , it can easily be shown that such a line should have a slope of $-E_{A} / k$. Thus, $E_{A}$ is calculated by taking the slope of the best fit line to the data and multiplying by $-k$.

Values for $E_{A}$ obtained using such methods are widely interpreted as having significance for dark current in digital imagers [32]-[35]. For instance, it is expected that the dark current activation energy, when generation-recombination centers in the 
depletion region dominates, will be measured to be approximately half the bandgap of intrinsic silicon. When diffusion from the neutral bulk dominates, the activation energy should be closer to the activation energy of the bandgap [23]. Using similar techniques, calculating energy levels of traps are used to characterize metal defects in CCDs [7]-[9].

We show in this work that such interpretation can be misleading for certain pixels with specific impurities. Some pixels have activation energy values dependent on signal charge. It is conjectured that these pixels contain an impurity near the edge of the depletion region when the region contains no signal charge. This model has been used previously to successfully duplicate properties of dark current production, including dark current as a function of exposure time [29], and suppression due to preillumination [31].

\subsection{Methods and results}

\subsubsection{Traditional activation energy calculations}

When calculating dark current activation energies, it is assumed that the plot of the natural $\log$ of counts versus the inverse of temperature will yield plots best characterized by a straight line as long as the pixel values do not approach saturation. However, we will show examples where the traditional Arrhenius plots at some exposure times and temperatures actually yield data that are not best characterized by a straight line. Data was taken with an SBIG ST-8XE camera with a KAF-1602E CCD sensor consisting of $1.56 \times 10^{6}$ pixels and a gain of $2.3 \mathrm{e}^{-} / \mathrm{DN}$. Examples of pixels 
with Arrhenius plots that are not best characterized as a linear relationship were observed in the KAF-1602E sensor as shown in Figs. 7.1b and 7.1c.
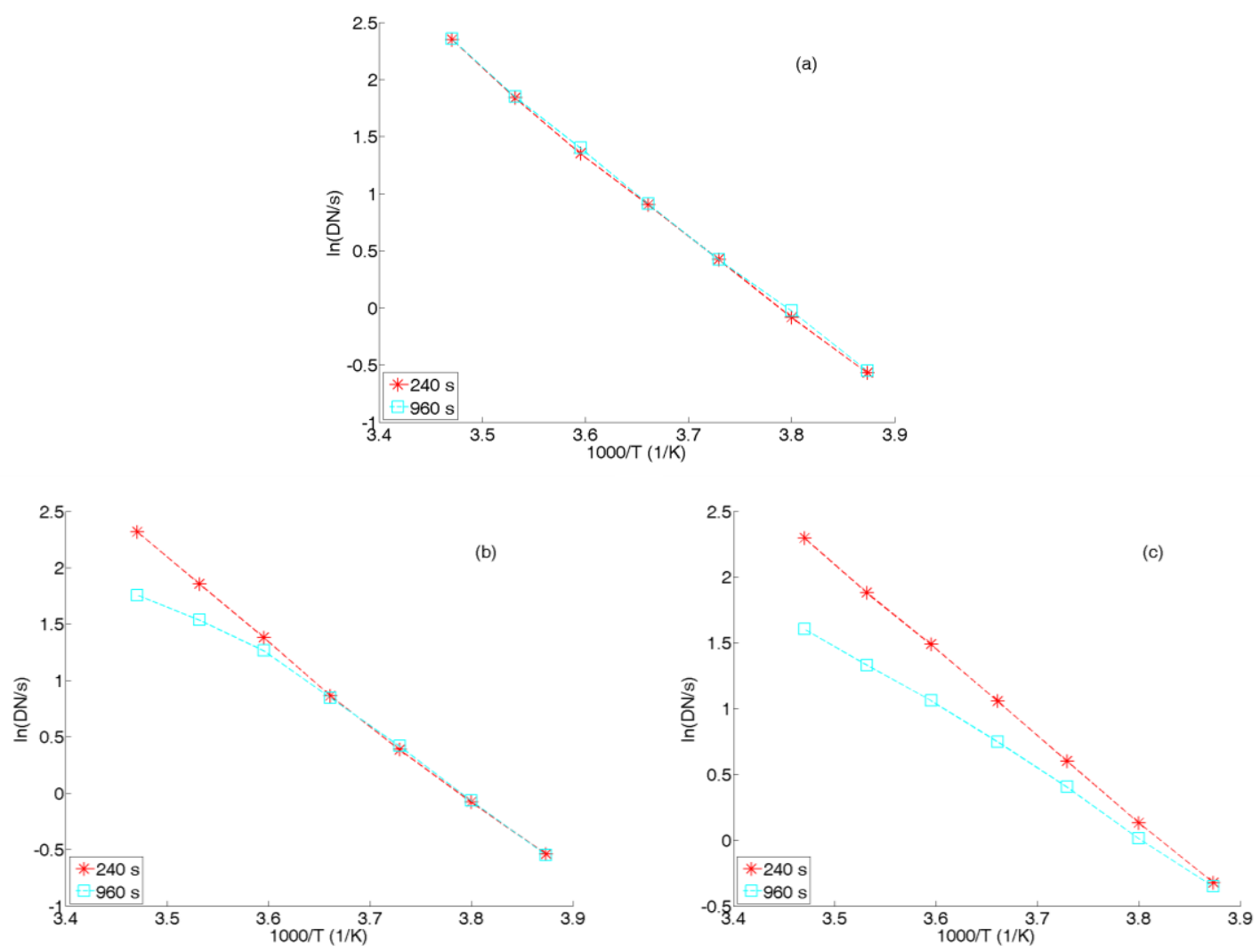

Fig. 7.1- Arrhenius plots of single hot pixels, including (a) a pixel showing no deviation from linearity at exposure times of $240 \mathrm{~s}$ and $960 \mathrm{~s}$, as well as (b) and (c) two pixels showing deviation from linearity at high temperatures with frames taken at an exposure time of $960 \mathrm{~s}$.

Traditional Arrhenius plots for three pixels from the KAF-1602E taken between $-15^{\circ} \mathrm{C}$ and $15^{\circ} \mathrm{C}$ are shown in Fig. 7.1. Plots are shown for each pixel taken with exposure times of both $240 \mathrm{~s}$ and $960 \mathrm{~s}$. Seen in Fig. 7.1a is a typical hot pixel, pixel $(1,880)$ on the sensor, with large dark current. This pixel is representative of most pixels in that the plot is linear regardless of the exposure time. The value calculated 
for the activation energy will be consistent regardless of whether it is calculated for $240 \mathrm{~s}$ or $960 \mathrm{~s}$ exposures. The pixel in Fig. $7.1 \mathrm{~b}$, pixel $(1,30)$, deviates from linearity at high temperatures for the Arrhenius plot at $960 \mathrm{~s}$, such that values are less than an extrapolated fit at lower temperatures would suggest, and therefore an activation energy calculated at these values will have a smaller value, $0.52 \mathrm{eV}$, compared to the $240 \mathrm{~s}$ value, $0.62 \mathrm{eV}$. The pixel shown in Fig. 7.1c $(10,407)$, deviates from linearity at even lower temperatures and therefore the deviation in calculated activation energies is even greater, $0.42 \mathrm{eV}$ for $960 \mathrm{~s}$ and $0.56 \mathrm{eV}$ for $240 \mathrm{~s}$. For ease of reference in the rest of this chapter, we will refer to the pixel in Fig. 7.1a as Pixel A, the pixel in Fig. 7.1b as Pixel B, and the pixel in Fig. 7.1c as Pixel C. These nonlinearities are not due to saturation as the DN values in these figures, the largest being about 5000 to $6000 \mathrm{DN}$, at $15 \mathrm{C}$ and $960 \mathrm{~s}$ for pixels B and C, are well below the saturation level of $65536 \mathrm{DN}$ for the Kodak imager.

From the plots in Figs. 7.1, it is observed that the traditional method of calculating activation energies is dependent on the chosen exposure time. This is troubling due to their importance associated in characterizing the dark current impurity sources. To further illustrate this dependency, data were taken at five exposure times from $240 \mathrm{~s}$ to $960 \mathrm{~s}$ and activation energies were calculated at each exposure time. As can be seen in Fig. 7.2, the activation energies for the two pixels showing deviation from linearity in Fig. 7.1, Pixels B and C, steadily decrease in value from relatively large activation energies at low exposure times to small activation energies when calculated at long 
exposure times. The pixel with a linear behavior in Fig. 7.1, Pixel A, shows a far more consistent value for the calculated activation energy across all exposure times.

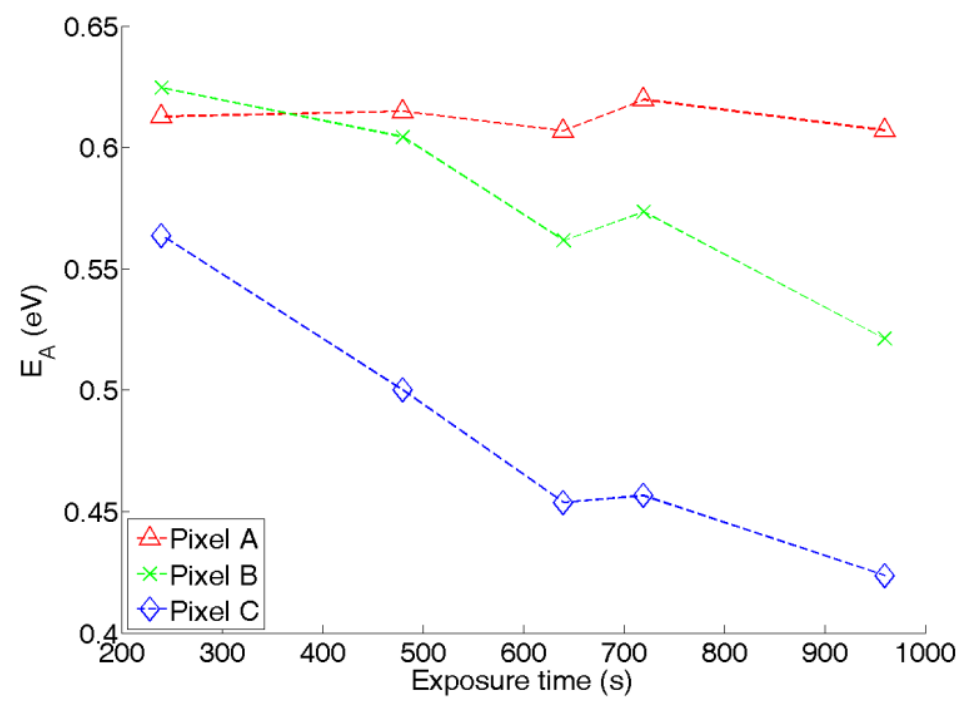

Fig. 7.2- Activation energies calculated at a series of exposure times for the KAF-1602E sensor.

\subsubsection{Activation energy exposure time dependency due to nonlinear production of current}

In prior studies, it has been found that dark current production of some pixels is diminished when exposed to light [17]. Additionally, smaller than expected dark frame values are measured at long exposure times when compared to extrapolated small exposure time dark frame values [26]. A model has been proposed that dark counts are reduced under these conditions due to fixed impurity locations and a moving depletion edge as the depletion region fills with collected charge resulting in fewer charges being collected due to thermal noise from the impurities [29], [31]. The variation in calculated activation energy could be due to the same phenomenon. Shown in Fig. 7.3 is the dark current at various exposure times for the three pixels from the Kodak imager shown in prior graphs. Pixel A has constant dark current regardless of 
exposure time. However, Pixels B and C produce significantly less dark current at longer exposure times than would be expected by extrapolating dark current production at short exposure times. It is likely then that the nonlinear Arrhenius plots for the two pixels is due to an increase in charge within the depletion region as an increase in temperature will result in more dark current production early in the collection time. The increase in collected charge will move the depletion edge and result in less charge collected due to thermal excitation from a fixed impurity that is no longer contained within the depletion region. The model that describes this phenomenon will be used later in the chapter to attempt to duplicate the behavior observed in the Kodak imager.

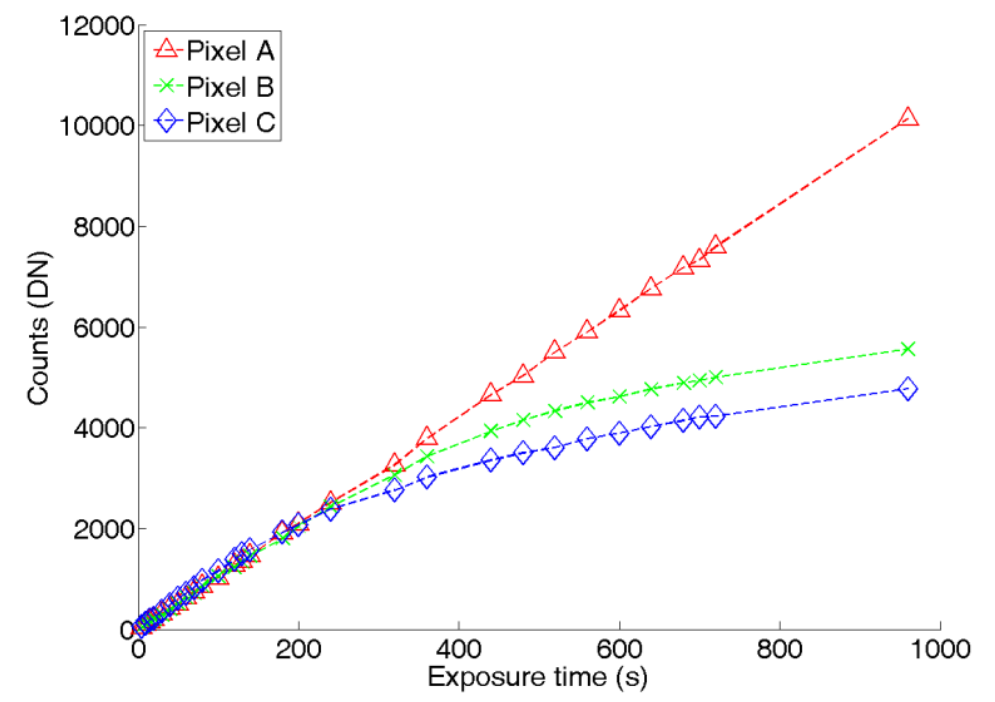

Fig. 7.3- Dark count at $288 \mathrm{~K}$ versus exposure time for the KAF$1602 \mathrm{E}$ sensor.

Due to the charge, and consequently digital count, dependency on the activation energy calculations, we suggest an alternative method to the traditional calculation. Instead of taking a series of frames at a single exposure time for the varying 
temperatures, an alternative is to take a series of frames such that a single count can be used across temperatures. A plot similar to the Arrhenius plot could be made such that rather than plotting the natural log of the counts at a constant exposure time as the $y$ axis, used instead is the natural log of the count, now kept constant, which has been divided by the exposure time where that count is obtained. Finding the slope of the line will again correspond to the activation energy and it can be calculated using the same procedure as the traditional method.

The exposure time to achieve a single count across all temperatures will vary pixel by pixel necessitating significantly more frames to be taken, an obvious disadvantage of this method. To generate the data to create Fig. 7.4, frames were taken at 33 exposure times for each of the temperatures, $-15^{\circ} \mathrm{C},-10^{\circ} \mathrm{C},-5^{\circ} \mathrm{C}, 0^{\circ} \mathrm{C}, 5^{\circ} \mathrm{C}, 10^{\circ} \mathrm{C}$, and $15^{\circ} \mathrm{C}$. Exposure times taken were chosen to attempt to maximize the amount of overlap in count values across all temperatures. For instance, frames with exposure times from $5 \mathrm{~s}$ up to $720 \mathrm{~s}$ were taken for $15^{\circ} \mathrm{C}$ while frames up to $46080 \mathrm{~s}$ were taken $-15^{\circ} \mathrm{C}$. Three dark frames, as well as three bias frames taken immediately before the dark frame, were collected for each exposure time. After the bias frames were subtracted, the average for each pixel was calculated for each exposure time to minimize dark current shot noise. In order to obtain a minimum of three data points for activation energy calculations, we selected the averaged frame at the exposure time for each temperature with the closest value to the chosen count with a maximum deviation of plus or minus ten percent. 


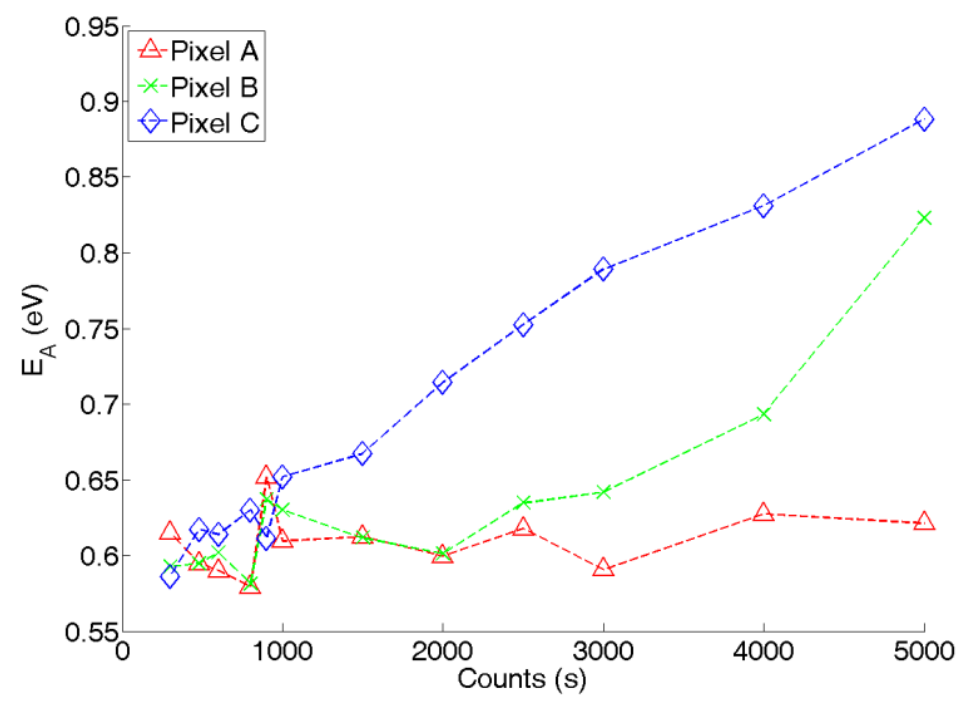

Fig. 7.4- Activation energy versus constant count values

In this way activation energies were calculated at a variety of count levels. Not surprisingly, the activation energies of Pixel A are fairly constant, at about half the bandgap of silicon, regardless of count level, with some expected deviation due to a variety of factors including the ten percent deviation allowed in selecting the exposure time. However, the activation energies of Pixels B and C are constant at low count levels but increase to larger values at larger counts.

To accurately characterize the dark current and impurity type based on activation energy, the count level therefore needs to be considered. Using the method of keeping a constant count instead of a constant exposure time, allows for choosing count levels where the activation energy is constant. Further, observing the change in activation energy values could provide insight into both the depletion and diffusion dark current behavior of the pixel. 


\subsection{Modeled activation energies due to a moving depletion edge}

A model based upon fixed impurity locations and a shrinking depletion region describes many aspects of observed dark current behavior including nonlinear generation with respect to exposure time and reduced generation after exposure to illumination [31], [36]. Another test of the model, as well as an opportunity for insight into impurity locations and behavior, presents itself in the observed dependency of calculated activation energy on count value.

Assigning different impurity locations, at a depth from the oxide layer, of either a single impurity or two impurities, has been demonstrated to reproduce the nonlinear production of dark current as seen in Fig. 7.5. Depths of the impurity or impurities for each pixel are given by the legend. The modeled pixel with the plot indicated by red circles has no impurity and therefore produces a small constant dark current. A pixel with an impurity that is not near the depletion edge, such as the pixel with an impurity at $3.96 \mu \mathrm{m}$, represented by orange asterisks, also produces a steady rate of dark noise up to the modeled $1000 \mathrm{~s}$. The edge of the depletion region was modeled to move from $5.33 \mu \mathrm{m}$ when empty to $3.93 \mu \mathrm{m}$ when full to simulate the conditions using parameters taken from the Kodak imager. Simulated pixels with impurities closer to the edge of the depletion region, such as the pixels with an impurity at $5.33 \mu \mathrm{m}, 5.27 \mu \mathrm{m}$, as well as $5.24 \mu \mathrm{m}$, are observed to produce less dark current after the depletion region moves past the impurity location. Electrons excited from thermal noise at the impurity location are not as likely to be collected by the depletion region once the impurity is 
no longer within the depletion region. For instance, the modeled pixel with an impurity at $5.33 \mu \mathrm{m}$, represented by light green dots, immediately begins to produce less dark current due to the impurity leaving the depletion region after the accumulation of charge. Therefore only a small fraction of the electrons produced by the impurity will be collected by the pixel's potential well. As the potential well fills with signal charge, the electrons produced by the impurity have a smaller chance of being collected.

Behavior of pixels with two impurities is even more complex. The pixel with two impurities, one close to the depth of the empty depletion region, $5.33 \mu \mathrm{m}$, and one closer to the oxide layer, $4.21 \mu \mathrm{m}$, produces enough dark current in the beginning of the simulated exposures to have just slightly more dark current than the pixel with a single impurity at $3.96 \mu \mathrm{m}$. A pixel with two impurities relatively close to the oxide layer, such as the pixel with two impurities at $4.91 \mu \mathrm{m}$ produce large amounts of dark current until they both move outside of the depletion region at about $700 \mathrm{~s}$. 


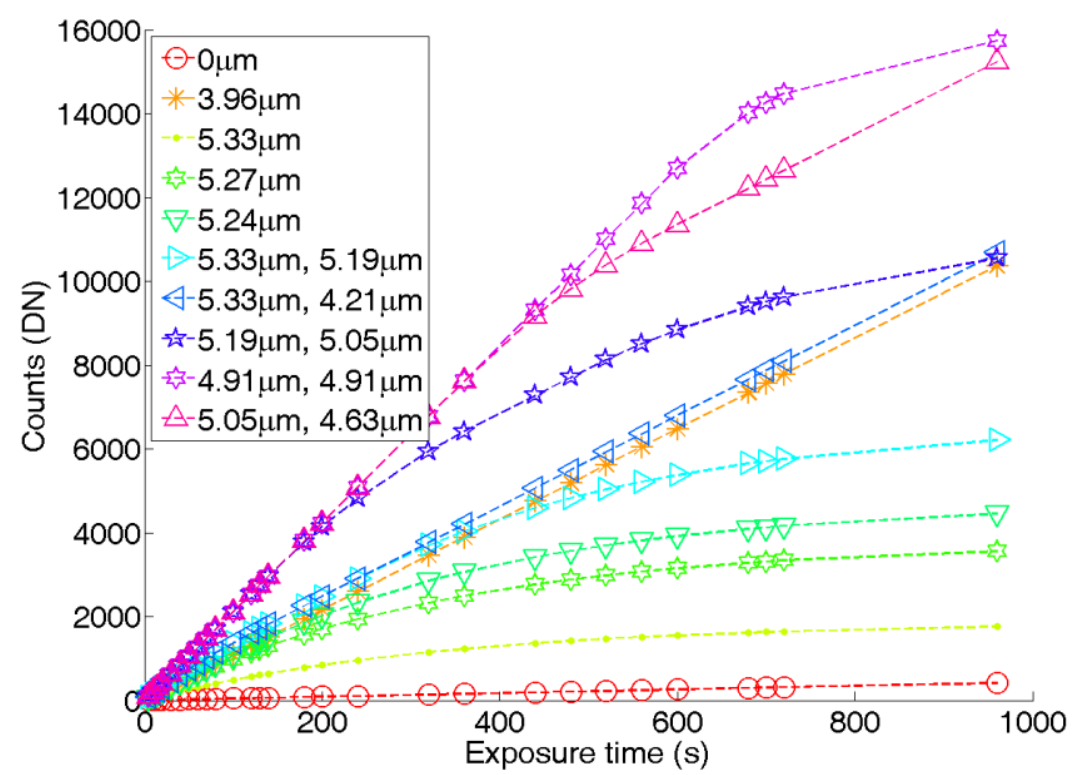

Fig. 7.5- Modeled counts vs. exposure time for simulated pixels with a moving depletion edge and fixed impurities. Locations of the impurities, as distance from the oxide layer, are given in the legend for each pixel.

Arrhenius plots were produced for the same simulated pixels and are presented in Fig. 7.6. A pixel with an impurity deep within the well, such as the one with an impurity at $3.96 \mu \mathrm{m}$, produces constant dark current amounts and the Arrhenius plot is observed to be linear, similar to Pixel A in Fig. 7.1a. However, the pixels with an impurity closer to the edge produce less dark count at higher temperatures than would be expected at the lower temperatures, such as Pixels B and C. To obtain the dark current produced by the simulated pixels at varying temperatures, a histogram was produced at each temperature for frames taken with the KAF-1602E sensor at long exposure times. For instance, Fig 3.3 could be used although the y-axis in that figure is not necessary for this purpose. Pixels with no impurities, or an impurity sufficiently far outside of the depletion region, were assigned the dark current of the main peak. 
The counts produced by the main peak were divided by the exposure time to obtain the dark current rates. Individual impurities were modeled with dark current rates of count production the same as the minor peak in the histogram. Pixels with two impurities were given twice that dark current while the impurities stayed within the depletion region and sufficiently far enough away from the depletion region.

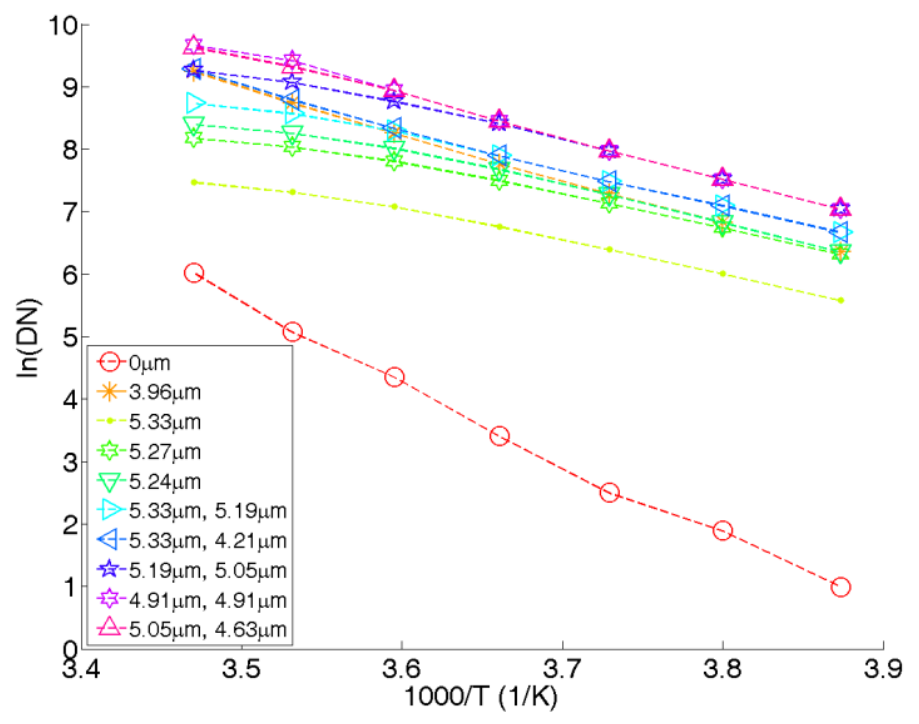

Fig. 7.6- Modeled Arrhenius plots with a 900 s exposure time

Activation energies were calculated for the modeled pixels from $240 \mathrm{~s}$ to $960 \mathrm{~s}$ and are displayed in Fig. 7.7. A drop off in activation energies are observed for the modeled pixels with nonlinear Arrhenius plots, but they stay constant at about half the bandgap of silicon for a pixel with an impurity fully within the depletion region. The modeled pixel with no impurity has constant activation energy at about the bandgap. 


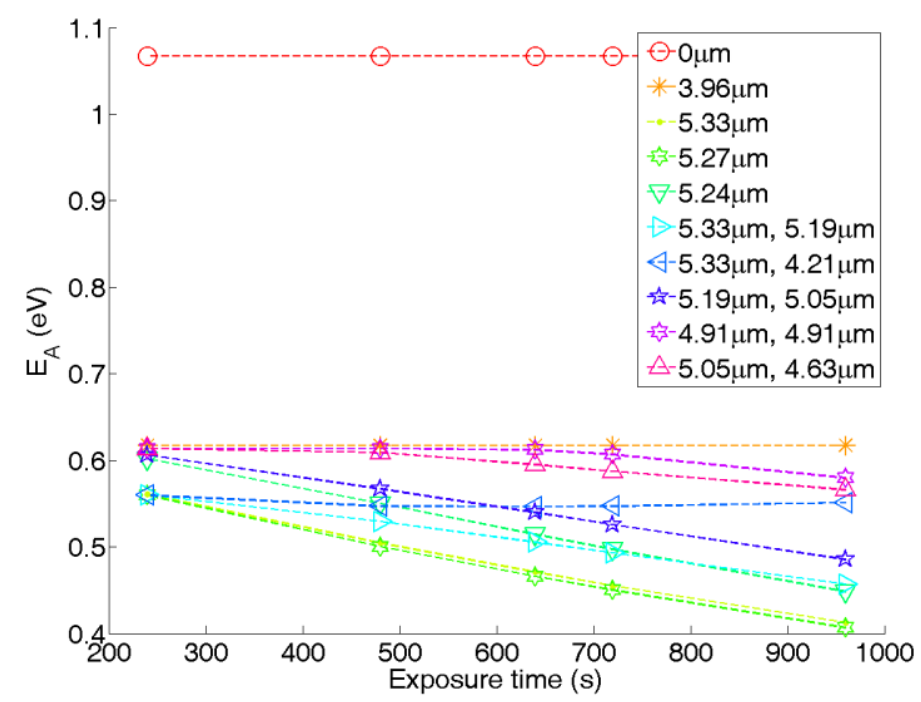

Fig. 7.7- Modeled calculated activation energies at varying exposure times

Arrhenius plots at a constant value were created using the modeled pixels; a constant value of $5000 \mathrm{DN}$ is used for the Arrhenius plots presented in Fig. 7.8. Linear behavior is observed for all of the pixels regardless of depth of the impurity present in the modeled pixel. This is to be expected as the count, and therefore distance between depletion edge and impurity, is constant across all temperatures. 


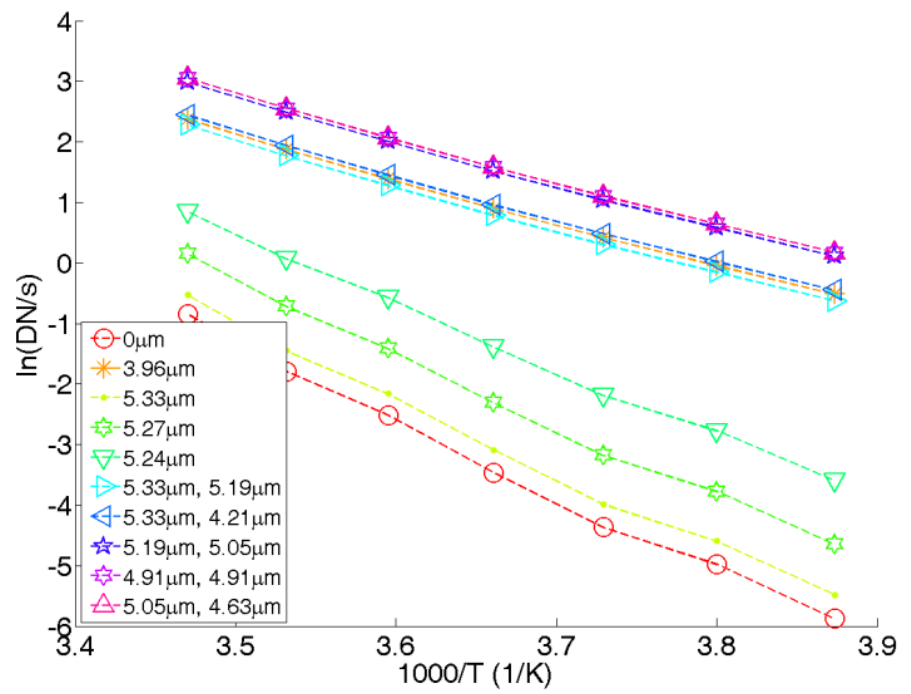

Fig. 7.8 Modeled Arrhenius plots with a fixed value of 5000 DN

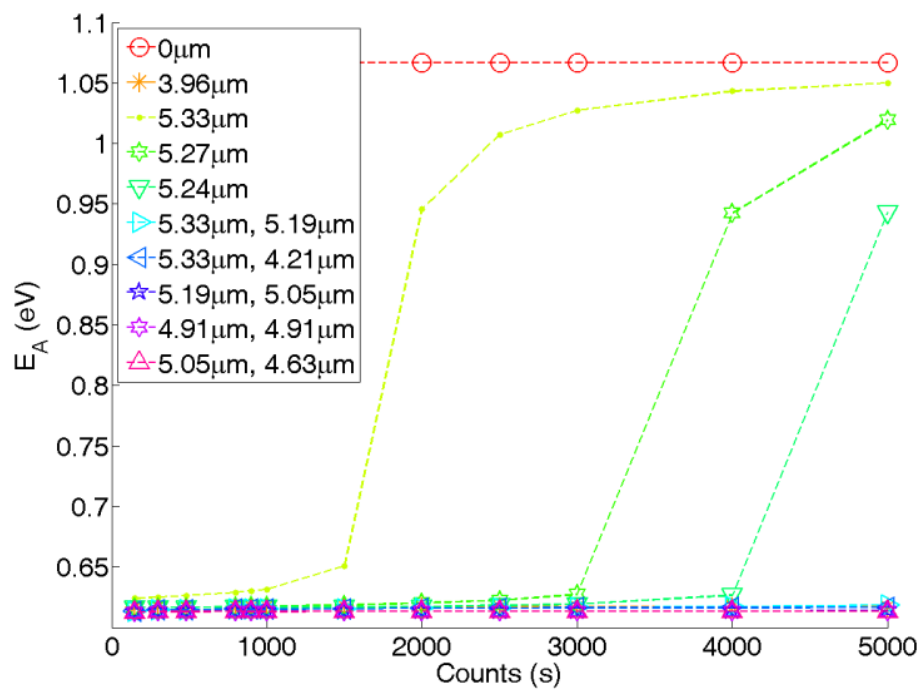

Fig. 7.9- Modeled calculated activation energies at varying fixed counts

Even though the Arrhenius plots are linear, the calculated activation energies will still depend on the chosen count value. This is a result of the maximum relative distance between impurity and depletion edge varying based on the count level chosen. However, a fixed count level will result in the maximum relative distance between 
impurity and depletion edge to be consistent. From Fig. 7.9 we see similar behavior for the pixels from the KAF-1602E sensor as seen in Fig. 7.4 where activation energies go from about half the bandgap of silicon at low count levels and, depending on the given depths of the impurity or impurities, increase and level off at about the bandgap. This behavior, in both the KAF-1602E and the model, suggests that the dark current transitions from an activation energy associated with depletion current, when the impurity is within the depletion region, to diffusion dark current, when the edge of the well moves past the impurity location.

\subsection{Conclusion}

Inconsistent activation energy values have been observed in digital imagers. In particular, for some pixels activation energies are dependent on the exposure time used in calculation. This inconsistency has been shown to be tied to the count level and therefore likely to the filling of the depletion region. A model describing the presence of impurities close to the boundary of a pixel's empty depletion region reproduces the inconsistent activation energy calculation. Choosing to instead use a consistent count

level, instead of the traditional consistent exposure time, controls for the variability of depletion edge depth and allows for choosing regimes that produce linear Arrhenius plots. 


\section{CHAPTER 8 - DISCUSSION AND CONCLUSIONS}

Pixels with nonlinear dark current generation with respect to exposure time have been observed in multiple digital imagers, including imagers of both CCD and CMOS pixel architectures. While nonlinear production has been widely observed previously close to saturation, these newly characterized pixels show nonlinear production well below saturation. Further, these pixels show a deviation from linear production in dark current at signal charge levels unique to each individual pixel and consistent from frame to frame despite changing variables such as exposure time and temperature. This dependency on signal charge has been demonstrated to exist independently of whether or not the signal charge has been generated by thermally excited charges or pre-illumination of the imager.

A model, incorporating a moving depletion edge with increasing signal charge as well as pixels with fixed impurity locations, has been successful in reproducing much of the observed nonlinear behavior of the pixels as well as their response to preillumination. The model predicts that pixels with fixed impurities with depths near the edge of the empty depletion region will largely shut off dark current production when the impurity or impurities leave the depletion region.

Activation energies are used extensively to characterize dark current sources and are conventionally expected to be invariant regardless of exposure time. A consequence of the pixels with a nonlinear dark current production is a dependency on signal charge 
for activation energy calculations. This dependency is observed in the empirical data taken from pixels showing nonlinear dark current production. Therefore, following traditional procedures of using a constant exposure time across all temperatures in calculations will result in an erratic value dependent on exposure time used. As signal charge increases with exposure time, at a given temperature, the model would suggest that an impurity near the edge of the empty depletion region would contribute significantly at smaller values, but would stop contributing significantly at greater values. Modeled pixel behavior reinforces this conclusion, accurately reproducing much of the observed activation energy calculations across diverse selected exposure time and count values. Therefore, we make the suggestion of a more dependable method of activation energy calculation using constant count at varying exposure times for each temperature.

Suggested future work would involve expanding the one-dimensional model of the shrinking depletion region to a more robust three-dimensional model to more accurately reproduce behaviors observed in the pixels. Furthermore, the nonlinear Arrhenius plots suggest potential for exploring the Meyer-Neldel rule with respect to obtaining inversion points as a result of investigators' selection bias. Finally, varying the gate voltages on an imager will provide for empirical results that could validate predictions made by the moving depletion region model. 


\section{REFERENCES CITED}

[1] A. J. P. Theuwissen, Solid-state imaging with charge-coupled devices. Dordrecht; Boston: Kluwer Academic Publishers, 1995.

[2] J. R. Janesick, Scientific charge-coupled devices. Bellingham, Wash: SPIE Press, 2001.

[3] O. Yadid-Pecht and R. Etienne-Cummings, Eds., CMOS imagers: from phototransduction to image processing. Boston: Kluwer Academic, 2004.

[4] J. Nakamura, Image sensors and signal processing for digital still cameras. Boca Raton, FL: Taylor \& Francis, 2006.

[5] S. Lim, "Characterization of noise in digital photographs for image processing," in Electronic Imaging 2006, 2006, p. 606900-60690O.

[6] R. D. McGrath, J. Doty, G. Lupino, G. Ricker, and J. Vallerga, "Counting of Deep-Level Traps Using a Charge- Coupled Device," IEEE Trans. Electron Devices, vol. ED-34, no. 12, pp. 2555-2557, 1987.

[7] W. C. McColgin, J. P. Lavine, J. Kyan, D. N. Nichols, and C. V. Stancampiano, "Dark current quantization in CCD image sensors," in Electron Devices Meeting, 1992. IEDM’92. Technical Digest., International, 1992, pp. 113-116.

[8] W. C. McColgin, J. P. Lavine, and C. V. Stancampiano, "Probing metal defects in CCD image sensors," Proc Mat Res Soc Symp, vol. 378, no. 713, 1995.

[9] W. C. McColgin, J. P. Lavine, C. V. Stancampiano, and J. B. Russell, "Deep-level traps in CCD image sensors," MRS Proc., vol. 510, p. 475, 1998.

[10] J. C. Dunlap, O. Sostin, R. Widenhorn, and E. Bodegom, "Dark current behavior in DSLR cameras," in Proc. SPIE, 2009, p. 72490N-72490N-12.

[11] J. C. Dunlap, E. Bodegom, and R. Widenhorn, "Characterization and correction of dark current in compact consumer cameras," in Proc. SPIE, 2010, p. 75360J-75360J-12.

[12] V. Goiffon, P. Magnan, F. Bernard, G. Rolland, O. Saint-Pé, N. Huger, and F. Corbière, "Ionizing radiation effects on CMOS imagers manufactured in deep submicron process," in Electronic Imaging 2008, 2008, pp. 681607-681607.

[13] O. Exner, "Determination of the Isokinetic Temperature," pp. 366-367, 1970.

[14] S. Arrhenius, "Über die Dissociationswarme und den Einfluss der Temperatur auf den Dissociationsgrad der Elektrolyte," Z. Für Phys. Chem., vol. 4, pp. 96$116,1889$.

[15] R. Widenhorn, L. Mündermann, A. Rest, and E. Bodegom, "Meyer-Neldel rule for dark current in charge-coupled devices," J. Appl. Phys., vol. 89, no. 12, p. 8179, 2001.

[16] W. von Meyer and H. Neldel, "Über die Beziehungen zwischen der Energiekonstanten und der Mengenkonstanten a in der Leitwerts Temperaturformel bei oxydischen Halbleitern.," Z. Für Tech. Phys., vol. 18, no. $588,1937$.

[17] R. Widenhorn, I. Hartwig, J. C. Dunlap, and E. Bodegom, "Influence of illumination on dark current in charge-coupled device imagers," J. Electron. Imaging, vol. 18, no. 3, p. 033015, Jul. 2009. 
[18] A. S. Grove, Physics and technology of semiconductor devices. New York: Wiley, 1967.

[19] C.-T. Sah, R. N. Noyce, and W. Shockley, "Carrier Generation and Recombination in P-N Junctions and P-N Junction Characteristics," Proc. IRE, pp. 1228-1243, 1957.

[20] R. N. Hall, "Electron-hole recombination in germanium," Phys. Rev., vol. 87, no. 2 , p. $387,1952$.

[21] W. Shockley and W. T. Read Jr, "Statistics of the recombinations of holes and electrons," Phys. Rev., vol. 87, no. 5, p. 835, 1952.

[22] S. M. Sze, Physics of semiconductor devices, 3rd ed. Hoboken, N.J: WileyInterscience, 2007.

[23] R. Widenhorn, M. M. Blouke, A. Weber, A. Rest, and E. Bodegom, "Temperature dependence of dark current in a CCD," in Electronic Imaging 2002, 2002, pp. 193-201.

[24] W. C. McColgin, J. P. Lavine, J. Kyan, D. N. Nichols, and C. V. Stancampiano, "Dark current quantization in CCD image sensors," in Electron Devices Meeting, 1992. IEDM'92. Technical Digest., International, 1992, pp. 113-116.

[25] R. Widenhorn, A. Rest, M. M. Blouke, R. L. Berry, and E. Bodegom, "Computation of dark frames in digital imagers," in Electronic Imaging 2007, 2007, pp. 650103-650103.

[26] R. Widenhorn, J. C. Dunlap, and E. Bodegom, "Exposure Time Dependence of Dark Current in CCD Imagers," IEEE Trans. Electron Devices, vol. 57, no. 3, pp. 581-587, Mar. 2010.

[27] R. Widenhorn, I. Hartwig, J. C. Dunlap, and E. Bodegom, "Measurements of dark current in a CCD imager during light exposures," in Proc. SPIE, 2008, vol. 6816, p. 681.

[28] "CMV2000 CMOS image sensor - CMOSIS - CMOS Image Sensors." [Online]. Available:

http://www.cmosis.com/products/standard_products/cmv2000. [Accessed: 02-Jun2014].

[29] J. C. Dunlap, M. M. Blouke, E. Bodegom, and R. Widenhorn, "Modeling Nonlinear Dark Current Behavior in CCDs," IEEE Trans. Electron Devices, vol. 59, no. 4, pp. 1114-1122, Apr. 2012.

[30] G. W. Taylor, P. K. Chatterjee, and H. H. Chao, "A Device Model for BuriedChannel CCD's and MOSFET's with Gaussian Impurity Profiles," IEEE Trans. ELECTRON DEVICES, vol. ED-27, no. 1, pp. 199-208, 1980.

[31] J. C. Dunlap, M. M. Blouke, E. Bodegom, and R. Widenhorn, "Dark current modeling with a moving depletion edge," J. Electron. Imaging, vol. 21, no. 4, pp. 043011-043011, 2012.

[32] N. S. Saks, "A technique for suppressing dark current generated by interface states in buried channel CCD imagers," Electron Device Lett. IEEE, vol. 1, no. 7, pp. 131-133, 1980. 
[33] D. K. Schroder, "The Concept of Generation and Recombination Lifetimes In Semiconductors," IEEE Trans. ELECTRON DEVICES, vol. ED-29, no. 8, pp. 1336-1338, Aug. 1982.

[34] H. I. Kwon, I. M. Kang, B.-G. Park, J. D. Lee, and S. S. Park, "The Analysis of Dark Signals in the CMOS APS Imagers From the Characterization of Test Structures," IEEE Trans. Electron Devices, vol. 51, no. 2, pp. 178-184, Feb. 2004.

[35] H. Rhodes, G. Agranov, C. Hong, U. Boettiger, R. Mauritzson, J. Ladd, I. Karasev, J. McKee, E. Jenkins, W. Quinlin, and others, "CMOS imager technology shrinks and image performance," in Microelectronics and Electron Devices, 2004 IEEE Workshop on, 2004, pp. 7-18.

[36] J. C. Dunlap, W. C. Porter, E. Bodegom, and R. Widenhorn, "Dark current in an active pixel complementary metal-oxide-semiconductor sensor," J. Electron. Imaging, vol. 20, no. 1, pp. 013005-013005, 2011. 